\title{
Irradiation Performance of HTGR Recycle Fissile Fuel
}

\author{
F. J. Homan \\ E. L. Long, Jr.
}

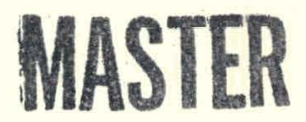

\section{OAK RIDGE NATIONAL LABORATORY}




\section{DISCLAIMER}

This report was prepared as an account of work sponsored by an agency of the United States Government. Neither the United States Government nor any agency Thereof, nor any of their employees, makes any warranty, express or implied, or assumes any legal liability or responsibility for the accuracy, completeness, or usefulness of any information, apparatus, product, or process disclosed, or represents that its use would not infringe privately owned rights. Reference herein to any specific commercial product, process, or service by trade name, trademark, manufacturer, or otherwise does not necessarily constitute or imply its endorsement, recommendation, or favoring by the United States Government or any agency thereof. The views and opinions of authors expressed herein do not necessarily state or reflect those of the United States Government or any agency thereof. 


\section{DISCLAIMER}

Portions of this document may be illegible in electronic image products. Images are produced from the best available original document. 
Printed in the United States of America. Available from National Technical Information Service

U.S. Department of Commerce

5285 Port Royal Road, Springfield, Virginia '2'2161

Price: Printed Copy $\$ 4.50$; Microfiche $\$ 2.25$

This report was prepared as an account of work sponsored by the United States Government. Neither the United States nor the Energy Research and Development Administration/United States Nuclear Regulatory Commission, nor any of their employees, nor any of their contractors, subcontractors, or their employees, makes any warranty, express or implied, or assumes any legal liability or responsibility for the accuracy, completeness or usefulness of any information, apparatus, product or process disclosed, or represents that its use would not infringe privately owned rights. 
ORNL/TM-5502

UC-77 (Gas-Cooled

Reactor Technology)

Contract No. W-7405-eng-26

METALS AND CERAMICS DIVISION

IRRADIATION PERFORMANCE OF HTGR RECYCLE FISSILE FUEL

F. J. Homan and E. L. Long, Jr.

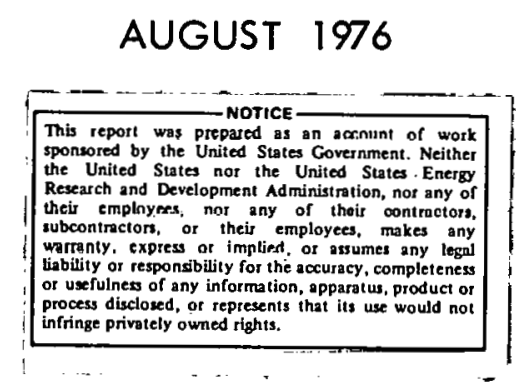

OAK RIDGE NATIONAL LABORATORY

Oak Ridge, Tennessee 37830

operated by

UNION CARBIDE CORPORATION

for the

ENERGY RESEARCH AND DEVELOPMENT ADMINISTRATION 
THIS PAGE

\section{WAS INTENTIONALLY \\ LEFT BLANK}


CONTENTS

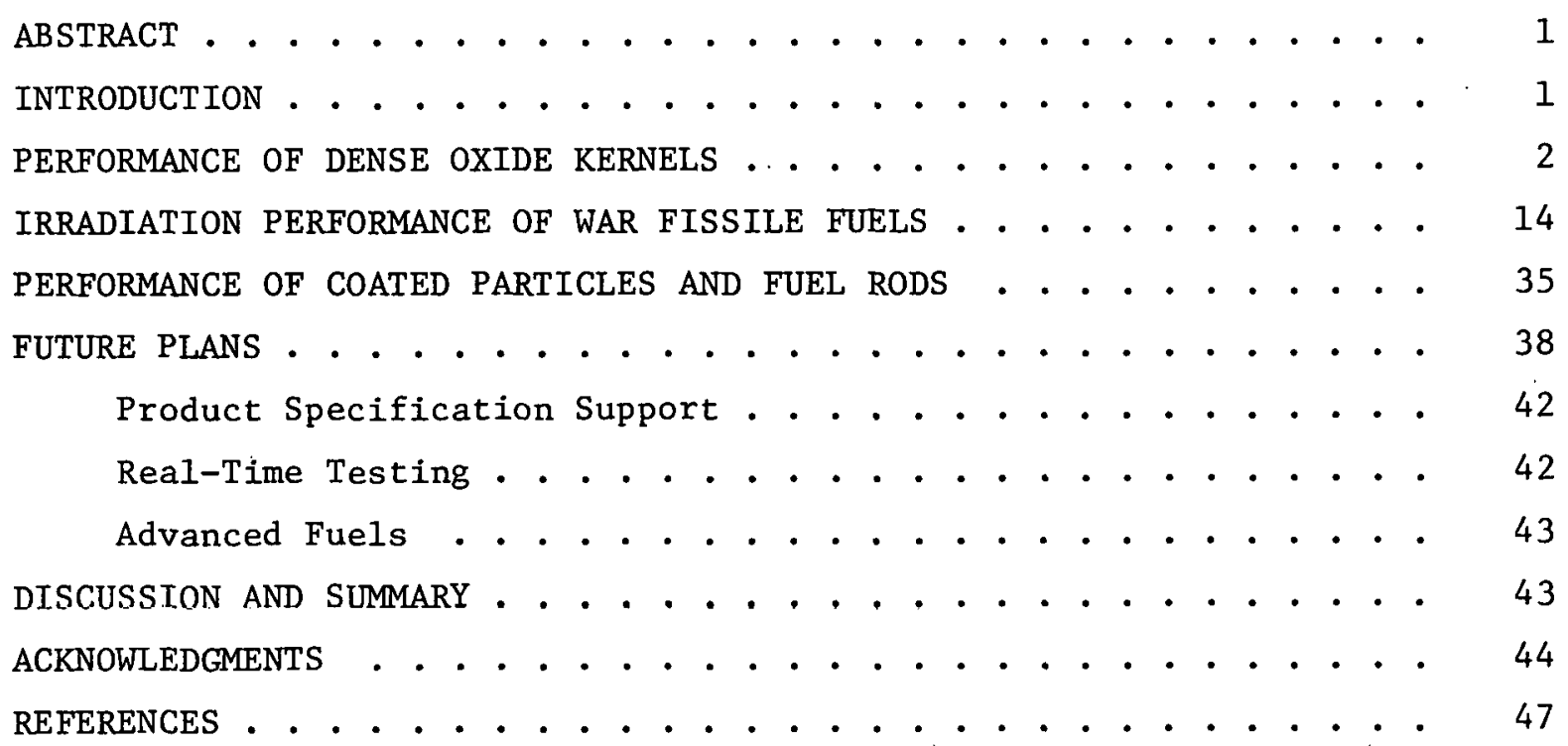


IRRADIATION PERFORMANCE OF HTGR RECYCLE FISSILE FUEL

F. J. Homan and E. L. Long, Jr.

\section{ABSTRACT}

The irradiation performance of candidate HTGR recycle fissile fuel under accelerated testing conditions is reviewed. Failure modes for coated-particle fuels are described, and the performance of candidate recycle fissile fuels is discussed in terms of these failure modes. The bases on which $\mathrm{UO}_{2}$ and $(\mathrm{Th}, \mathrm{U}) \mathrm{O}_{2}$ were rejected as candidate recycle fissile fuels are outlined, along with the bases on which the weak-acid resin (WAR)-derived fissile fuel was selected as the reference recycle kernel. Comparisons are made relative to the irradiation. behavior of WAR-derived fuels of varying stoichiometry and conclusions are drawn about the optimum stoichiometry and the range of acceptable values. Plans for future testing in support of specification development, confirmation of the results of accelerated testing by real-time experiments, and improvement in fuel performance and reliability are described.

\section{INTRODUCTION}

Irradiation testing of fissile fuels in support of HTGR recycle fuel development ${ }^{1}$ has involved three distinct kernel types. They are (1) $\mathrm{UO}_{2}$, (2) $\left(\mathrm{Th}_{1-z}-\mathrm{U}_{z}\right) \mathrm{O}_{2}$, and (3) a uranium-containing kernel derived from a weak-acid ion exchange resin. This testing was done over a ten-year period and is summarized in ref. 2. This derade of teeting has resulled in the identification of five failure mechanisms for coated-particle fuels:

1. "Amoeba" or thermal migration of the kernel up the temperature gradient and through the coating layers.

2. Fission-product attack of the coatings and mechanical interaction of coatings with fission products, usually in combination, which results in ruptured coatings. This problem is most severe in Triso particles, where the SiC layer is attacked.

3. Pressure vessel failure früu Internal gas pressure and stresses caused by the dimensional changes in the coating, resulting from irradiation damage.

4. Matrix-particle interaction involving a strong bond between the outer coating and the matrix material. Both materials densify under irradiation, resulting in tensile forces that will destroy coating integrity if the bond is not broken first. 
5. Fabrication defects or imperfections in the coatings introduced during coating or in subsequent processing.

Of the failure mechanisms listed above, thermal migration and fission product attack of the coatings are related to kernel chemistry. Thermal migration eliminated both $\mathrm{UO}_{2}$ and $\left(\mathrm{Th}_{1-z} \mathrm{U}_{z}\right) \mathrm{O}_{2}$ from consideration as fissile recycle fuel. Fission-product attack of the SiC coating eliminated WAR-derived $\mathrm{UC}_{2}$ kernels. Dense $\mathrm{UC}_{2}$ kernels were not considered for recycle application, because kernel preparation processes were considered to be too complex for remote operation. Pressure vessel failure, matrix-particle interaction, and fabrication defect failure are not related to kernel chemistry.

This report will discuss only irradiation testing of candidate recycle fissile fuels. Work on process considerations; economics, and thermodynamic analysis is reported elsewhere. ${ }^{1}$ Most of the discussion on irradiation performance is associaced wilh coated porticles, and, in particular, the kernels. Most of the testing described is with fuel rods made by the slug-injection ${ }^{3}$ process, and some aspects of fuel rod performance will be covered.

\section{PERFORMANCE OF DENSE OXIDE KERNELS}

The first candidate recycle fuel for the HTGR was $\mathrm{UO}_{2}$. Early irradiation testing of $\mathrm{UO}_{2}$ Identified a severe problem with thermal migration of the $\mathrm{UU}_{2}$ keruel up the tcmpcrature gradient and through the coating layers. An example of this migration is shown in Fig. 1 . The temperature and temperature gradient to which these particles were crpoesd were nearly equivalent to those in a targe High-Temperature Cas-Cooled Rejactor (LHTGR). Representative HTGR temperatures and temperature gradients are provided in Fig. 2 and l'able $1.4,5$

The thermal stability of $\mathrm{UO}_{2}$ relative to $\mathrm{ThO}_{2}$ can be seen in Fig. 1 . The subject of thermal mig ration has been extcncively stildied and documented in the technical literature ${ }^{6,7}$ and will not be discussed in depth in this report. Figure 3 compares the average thermal stability of several. kernels, among them $\mathrm{UO}_{2}$ and $\mathrm{ThO}_{2}$; the upper and $1.0 w e r 90 \%$ confidence limits for the data generally lie half an order of magnitude above and below the average. The cross-hatched region and above represents unacceptable performance. This region was determined by calculating the kernel migration coefficients that would lead to migration of the kernel through the buffer layer during normal operation, for different regions of an LHTGR core. Figure 3 shows that the $\mathrm{UO}_{2}$ curve intersects the cross-hatched region in the temperature range of primary interest for the LHTGR, whereas the $\mathrm{ThO}_{2}$ curve is well below the cross-hatched area for all temperatures. The $\mathrm{UO}_{2}$ kernels were therefore rejected from the development program. 

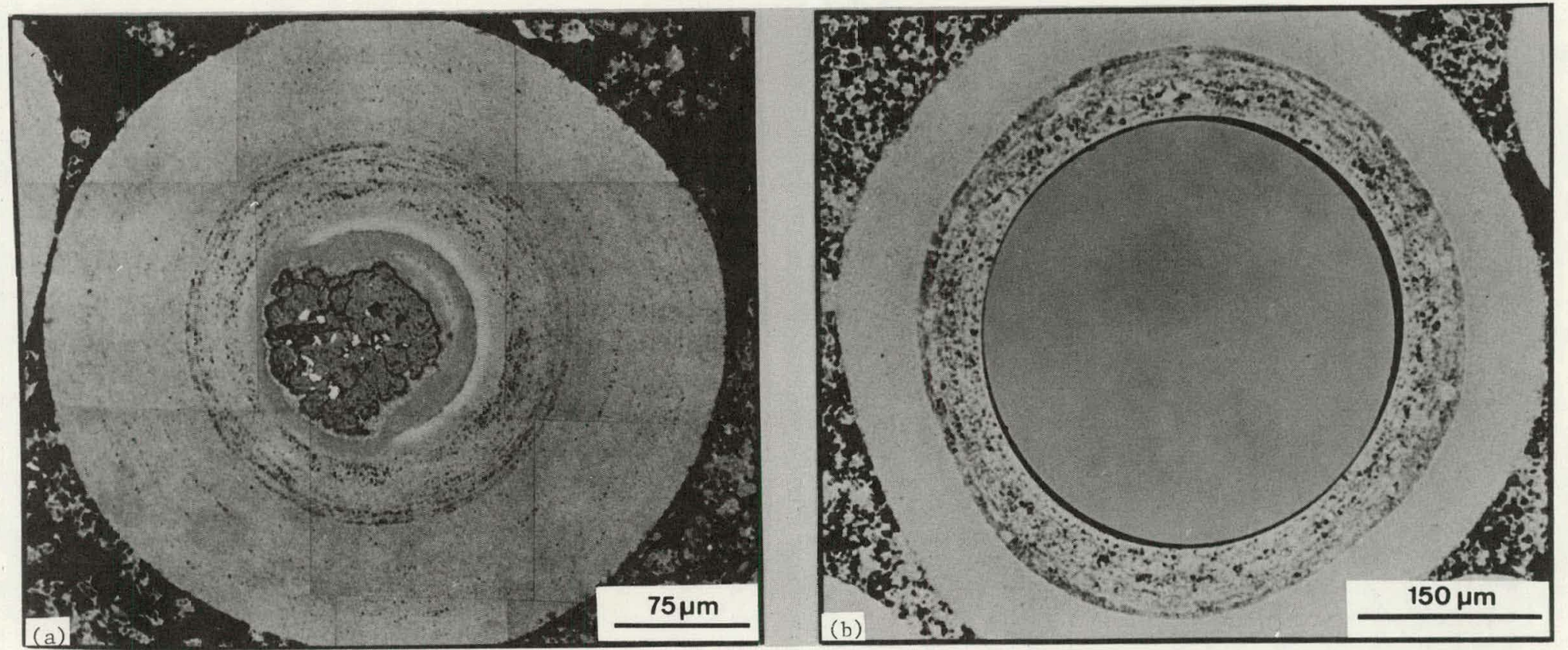

Fig. 1. Relative Thermal Stability of Fissile and Fertile Kernels from Irradiation Test $\mathrm{H}-1-2$. Temperature, $950^{\circ} \mathrm{C}$; thermal gradients, $1000^{\circ} \mathrm{C} / \mathrm{cm}$; fast fluences, $5 \times 10^{21} \mathrm{n} / \mathrm{cm}^{2}$; time at power, 97 days. (a) $\mathrm{UO}_{2}(33 \% \mathrm{FIMA})$. (b) $\mathrm{ThO}_{2}(0.2 \% \mathrm{FTMA})$. 


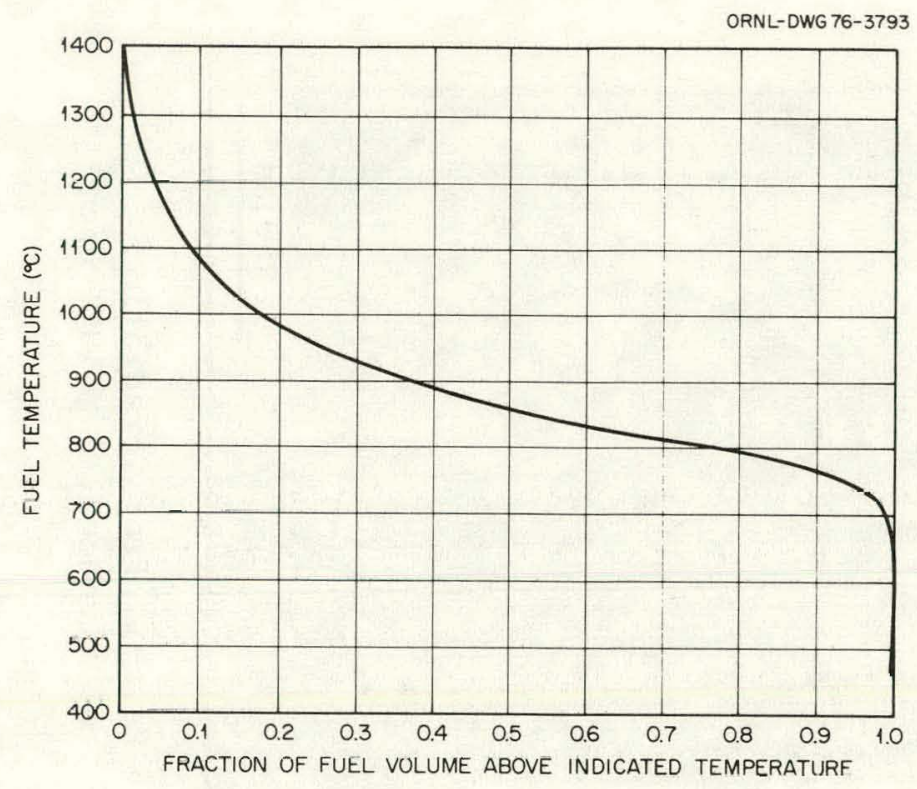

Fig. 2. Fraction of Fuel Operating Above a Given Temperature Under Normal LHTGR Conditions.

Table 1. Comparison of Temperature and Heat Production Parameters for Three Nuclear Power Stations

\begin{tabular}{|c|c|c|c|}
\hline & Ft. St. Vrain & Summil SLation & Fulton station \\
\hline I'hermal power (MW) & 842 & 2000 & 30000 \\
\hline Power density (kW/liter) & 6.3 & 8.1 & 8.4 \\
\hline \multicolumn{4}{|l|}{ Fuel rod heat rate $(\mathrm{kW} / \mathrm{ft})$} \\
\hline Average & 1.2 & 2.3 & 2.4 \\
\hline Maximum & 3.8 & 6.8 & 7.0 \\
\hline \multicolumn{4}{|c|}{ Heat flux at coolant holes $\left(\mathrm{W} / \mathrm{cm}^{2}\right)$} \\
\hline Average & 14.2 & 20.5 & 20.5 \\
\hline Maximum & 44.2 & 57.4 & 58.4 \\
\hline \multicolumn{4}{|l|}{ Fue1 temperatures $\left({ }^{\circ} \mathrm{C}\right)$} \\
\hline Volume average & 810 & $85 /$ & 890 \\
\hline Maximum (short term) & 1260 & 1420 & 1410 \\
\hline Hot channel & & 1585 & 1571 \\
\hline \multicolumn{4}{|l|}{ Graphite temperatures $\left({ }^{\circ} \mathrm{C}\right)$} \\
\hline Average & 743 & 777 & 739 \\
\hline Maximum & 1038 & 1121 & 1142 \\
\hline \multicolumn{4}{|c|}{ Temperature gradients $\left({ }^{\circ} \mathrm{C} / \mathrm{cm}\right)^{\mathrm{a}}$} \\
\hline Average & & 240 & 250 \\
\hline Maximum & & 670 & 700 \\
\hline
\end{tabular}

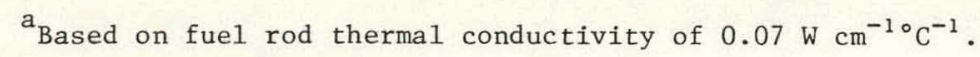




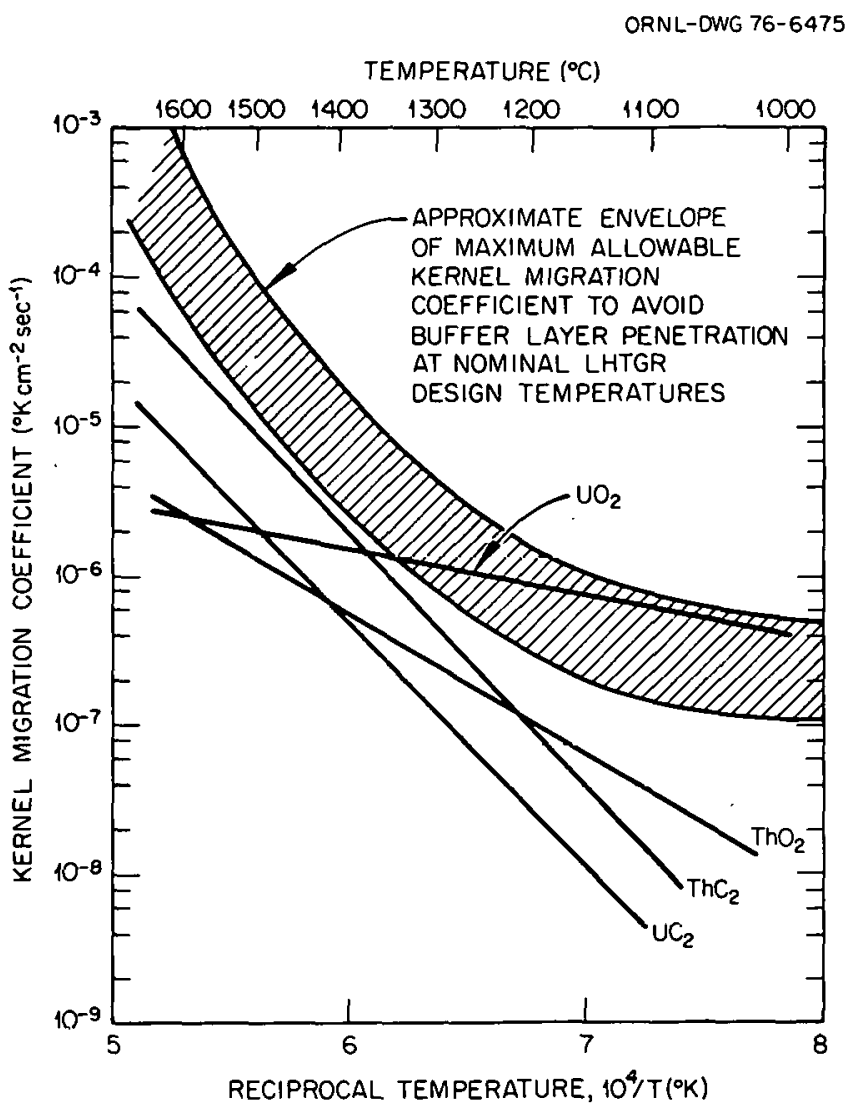

Fig. 3. Average Fuel Kernel Migration Coefficients vs Inverse Temperature Determined From In- and Out-of-Reactor Experiments on Fully Dense Kernels. From D. P. Harmon and C. B. Scott, Development and Irradiation Performance of LHTGR Fuel, GA-Al3173 (October 1975), p. 103.

The favorable performance of $\mathrm{ThO}_{2}$ relative to $\mathrm{UO}_{2}$ suggested dilution of $\mathrm{UO}_{2}$ with enough $\mathrm{ThO}_{2}$ for stabilization. Since the overall $\mathrm{Th} / \mathrm{U}$ ratio in a recycle fuel element is about 8 , it appeared as though considerable latitude existed. Mixed thorium-uranium oxide in the ratio of about 4:1 was easy to prepare, and the process was thought to be readily adaptable to the remote operation required in HTGR fuel refabrication. The $\left(\mathrm{Th}_{0}{ }_{6} \mathrm{U}_{0} \mathrm{C}_{2}\right)_{\mathrm{O}_{2}}$ kernel was selected as the reference recycle fissile fue $1^{8}$ to replace $\mathrm{UO}_{2}$, and an irradiation program to test the new reference was begun.

A summary of the irradiation testing of the dense mixed-oxide recycle particle is contained in Table 2. Stoichiometries from $\mathrm{Th} / \mathrm{U}=1$ to $\mathrm{Th} / \mathrm{U}=8$ have been tested. Early results at modest irradiation temperatures were encouraging, as shown in Fig. 4 (ref.9). Very little migration of the ( $\left.\mathrm{Th}_{0 .}{ }_{8} \mathrm{U}_{0.2}\right)_{\mathrm{O}_{2}}$ has occurred, and as before, the $\mathrm{ThO}_{2}$ is very stable. Unfortunately, at higher operating temperatures the $\left(\mathrm{Th}_{0.8} \mathrm{U}_{0.2}\right)_{2}$ was unstable [Fig. 5 (ref. 10)] where the kernel had migrated through the coatings. Table 2 shows that a significant amount of irradiation data on dense mixed-oxide kernels has been accumulated. 
Table 2. Summary of ORNL Experimental Data on Mixed (Th,U) Oxide Recycle Fuel Part 1. Real-Time Testing Under HTGR Conditions in Peach Bottom Reactor

\begin{tabular}{|c|c|c|c|c|c|c|c|c|}
\hline $\begin{array}{l}\text { Irradiation }(1,2) \\
\text { experiment } \\
\text { No. }\end{array}$ & $\begin{array}{l}\text { Batch No. } \\
\text { and } \\
\text { fuel type }\end{array}$ & $\begin{array}{l}\mathrm{Th} / \mathrm{u} \\
\text { rat1o }\end{array}$ & $\begin{array}{l}\text { Fast fluence } \\
\mathrm{E}>0.18 \mathrm{MeV} \\
\left(\mathrm{n} / \mathrm{cm}^{2}\right)\end{array}$ & $\begin{array}{l}\text { Average } \\
\text { burnup } \\
\text { ( } X \text { FIMA) }\end{array}$ & $\begin{array}{l}\text { Maximum } \\
\text { fuel } \\
\text { temperature } \\
\left({ }^{\circ} \mathrm{C}\right)\end{array}$ & $\cdot{ }_{(\mu \mathrm{a})}^{\operatorname{Desi}^{a}}$ & $\begin{array}{l}\text { Dens } 1 \text { tyy } \\
\left(\mathrm{g} / \mathrm{cm}^{3}\right)^{\mathrm{a}}\end{array}$ & Renarks \\
\hline \multicolumn{9}{|l|}{ RTE-1 (FTE-11) } \\
\hline Body 1 & $\begin{array}{l}\mathrm{PR}-56 \\
\mathrm{PR}-66\end{array}$ & $\begin{array}{l}4: 1 \\
2: 1\end{array}$ & $3.3 \times 10^{21}$ & $\begin{array}{r}9.0 \\
14.7\end{array}$ & c & $\begin{array}{l}350 / 75 / 130 \\
350 / 90 / 130\end{array}$ & $\begin{array}{l}10.1 / 1.1 / 1.9 \\
10 / 1.17 / 1.86\end{array}$ & $\begin{array}{l}\text { Slug-injected; carbonized in covered } \\
\text { graphite tray; to be examined. }\end{array}$ \\
\hline Kody 2 & $\begin{array}{l}P R-56 \\
P R-66\end{array}$ & $\begin{array}{l}1: 1 \\
2: 1\end{array}$ & & $\begin{array}{r}9.0 \\
14.7\end{array}$ & & $\begin{array}{l}35 n / 75 / 130 \\
350 / 90 / 130\end{array}$ & $\begin{array}{l}10.1 / 1.1 .1 .9 \\
10 / 1.17 / 1.86\end{array}$ & \\
\hline Budy 3 & $\begin{array}{l}\begin{array}{l}P k=36 \\
\mathrm{PR}-67\end{array} \\
\end{array}$ & $\begin{array}{l}4: 1 \\
2: 1\end{array}$ & ‘ & $\begin{array}{r}-9.0 \\
14.7\end{array}$ & & $\begin{array}{l}350 / 75 / 130 \\
350 / 90 / 140\end{array}$ & $\begin{array}{l}10.1 / 1.1 / 1.9 \\
10 / 1.17 / 1.85\end{array}$ & 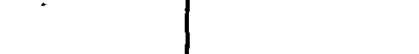 \\
\hline Body 4 & $\begin{array}{l}\text { PR-56 } \\
\text { PR-67 }\end{array}$ & $\begin{array}{l}4: 1 \\
2: 1\end{array}$ & & $\begin{array}{r}9.0 \\
14.7\end{array}$ & & $\begin{array}{l}350 / 75 / 130 \\
350 / 90 / 140\end{array}$ & $\begin{array}{l}10.1 / 1.1 / 1.9 \\
10 / 1.17 / 1.85\end{array}$ & $\cdot$ \\
\hline Body 5 & PR-66 & $2: 1$ & & 14.7 & & $350 / 90 / 130$ & $10 / 1.17 / 1.86$ & \\
\hline Bodỹ b & PR-66 & $2: 1$ & & 14.7 & 1 & $350 / 90 / 130$ & $10 / 1.17 / 1.86$ & \\
\hline RTE-2 & & & &. & & & & \\
\hline Body 2 & $P R-66$ & $2: 1$ & $3.6 \times 20^{21}$ & .23 .3 & $1190^{d}$ & $350 / 90 / 130$ & $10 / 1.17 / 1.86$ & 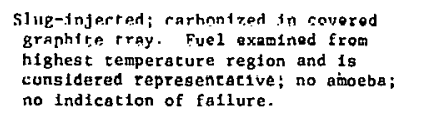 \\
\hline Body 5 & PR-55 & $4: 1$ & $3.6 \times 10^{21}$ & 8.2 & $1300^{d}$ & $350 / 70 / 80$ & $10.1 / 1.1 / 1.9$ & $\begin{array}{l}\text { Loose bed of particles; fuel examined } \\
\text { from highest temperature reglon and } \\
\text { ts considered representative; no } \\
\text { amoeba; no indication of fallure. }\end{array}$ \\
\hline \multicolumn{9}{|l|}{ RTE-4 } \\
\hline Body 3 & $\mathrm{Pn}-54$ & $4: 1$ & $2.0 \times 10^{21}$ & 5.0 & $1260^{d}$ & $350 / 70 / 70$ & $10.1 / 1.1 / 1.94$ & $\begin{array}{l}\text { Loose bed of particles; fuel examined } \\
\text { trom highest temperature region and } \\
\text { is considered representative; no } \\
\text { amoeba; no indications of failure. }\end{array}$ \\
\hline Bedy 5 & Pn 61 & $2: 1$ & $2.0 \times 10^{21}$ & B. 5 & $1230^{2}$ & $350 / 55 / 120$ & $10.2 / 1.16 / 1.91$ & $\begin{array}{l}\text { Slug-injected; carbonlzed in covered } \\
\text { graph1te Liay; fuel examined from } \\
\text { h1 ghest tomporaturo rcgion and is } \\
\text { considered represpentatiug: no }\end{array}$ \\
\hline RTE-S & 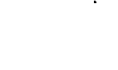 & & & $\cdot$ & 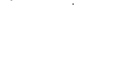 & 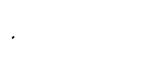 & & gaogba: no Indiration of fathiura. \\
\hline Rndy 1 & $\begin{array}{l}\text { PR-57-1 } \\
\text { PR-61 }\end{array}$ & $\begin{array}{l}4: 1 \\
2: 1\end{array}$ & $4.6 \times 10^{21}$ & $\begin{array}{l}11.9 \\
18.0\end{array}$ & i & $\begin{array}{l}350 / 75 / 135 \\
350 / 75 / 120\end{array}$ & $\begin{array}{l}10.1 / 1.1 / 1.9 \\
10.2 / 1.10 / 1.9\end{array}$ & 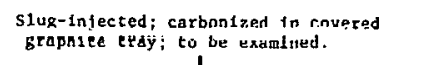 \\
\hline Body 2 & $\begin{array}{l}P R-57-1 \\
P R-61\end{array}$ & $\begin{array}{l}4: 1 \\
2: 1\end{array}$ & & $\begin{array}{l}1.1 .9 \\
18.4\end{array}$ & & $\begin{array}{r}350 / 75 / 135 \\
350 / 75 / 120\end{array}$ & $\begin{array}{l}10.1 / 1.1 / 1.9 \\
10.2 / 1.16 / 1.9\end{array}$ & \\
\hline Body 3 & $\begin{array}{l}\text { PR-57-1 } \\
\text { PR-61 }\end{array}$ & $\begin{array}{l}4: 1 \\
2: 1\end{array}$ & & $\begin{array}{l}21.9 \\
18.4\end{array}$ & . & $\begin{array}{l}350 / 75 / 135 \\
350 / 75 / 120\end{array}$ & $\begin{array}{l}10.111 .1 / 1.9 \\
10.2 / 1.16 / 1.9\end{array}$ & $\cdot$ \\
\hline Body 4 & $\begin{array}{l}\text { PR-57-I } \\
\text { DP-61 }\end{array}$ & $\begin{array}{l}4: 1 \\
2: 1\end{array}$ & & $\begin{array}{l}11.9 \\
18.4\end{array}$ & & $\begin{array}{l}350 / 75 / 135 \\
350 / / 5 / 120\end{array}$ & $\begin{array}{l}10.1 / 1.1 / 1.9 \\
10.2 / 1.16 / 1.9\end{array}$ & \\
\hline Body 5 & $P R-60$ & $2: 1$ & & 18.4 & & $350 / 75 / 120$ & $10.2 / 1.16 / 1.9$ & ·' \\
\hline Randy $A$ & $\mathbf{m n} \cdot 60$ & $\varepsilon: 1$ & & IN., & 1 & $350 / 75 / 120$ & $10,2 / 1.10 / 1.9$ & 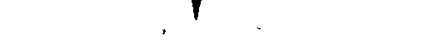 \\
\hline \multicolumn{9}{|l|}{ RTE-6 } \\
\hline Eody 3 & $P R-60$ & $2: 1$ &,$^{4.6 \times 10^{21}}$ & $\begin{array}{l}18.2 \\
.\end{array}$ & c & $350 / 75 / 120$ & $\begin{array}{c}10.2 / 1.16 / 1.9 \\
. \\
.\end{array}$ & 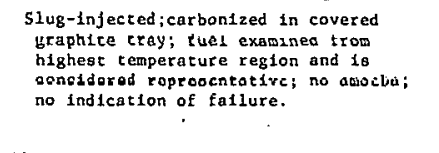 \\
\hline Burl, 4 & $p n=d u$ & $2 ! 1$ & $4.6 \times 10^{81}$ & iв. 1 & c & $350 / 75 / 120$ & $10.2 / 1.16 / 1.9$ & $\begin{array}{l}\text { Slug-infected; corbonized in covered } \\
\text { graphite tray. }\end{array}$ \\
\hline \multicolumn{9}{|l|}{ RTE-7 } \\
\hline Body 1 & PR-60 & $2: 1$ & $1.3 \times 10^{21}$ & 5.5 & $880^{d}$ & $350 / 75 / 120$ & $10.2 / 1.16 / 1.9$ & \multirow{4}{*}{$\begin{array}{l}\text { Slug-infected; carbonized in covered } \\
\text { graphite tray; [utl uxamined from highest } \\
\text { temperature region and is considered } \\
\text { representative; no smoeba; no indications } \\
\text { îf fullure. }\end{array}$} \\
\hline Body 2 & $P R-6 n$ & $2: 1$ & & 5.5 & $1130^{\circ}$ & $330 / / 2 / 120$ & $10.2 / 1.16 / 1.9$ & \\
\hline $\begin{array}{l}\text { Body } 3 \\
\text { Ansty } 6\end{array}$ & $\begin{array}{l}\text { PR-60 } \\
\text { Pn } 60\end{array}$ & $\begin{array}{l}2: 1 \\
2: 1\end{array}$ & & $\begin{array}{l}5.5 \\
5.5\end{array}$ & $\begin{array}{l}2230^{d} \\
1230^{A}\end{array}$ & $\begin{array}{l}350 / 75 / 120 \\
350 / 75 / 120\end{array}$ & $\begin{array}{l}10.2 / 1.16 / 1.9 \\
10.2 / 1.16 / 1.9\end{array}$ & \\
\hline Body 5 & $\begin{array}{l}P R-57-1 \\
P R-57-6 \\
P R-61\end{array}$ & $\begin{array}{l}4: 1 \\
4: 1 \\
2: 1\end{array}$ & & $\begin{array}{l}3.2 \\
3.2 \\
5.5\end{array}$ & $\begin{array}{l}1150^{\mathrm{d}} \\
1150^{\mathrm{d}} \\
1150^{\mathrm{d}}\end{array}$ & $\begin{array}{l}350 / 15 / 135 \\
350 / 75 / 135 \\
350 / 75 / 120\end{array}$ & $\begin{array}{l}10.1 / 1.1 / 1.9 \\
10.1 / 1.1 / 1.9 \\
10.2 / 1.16 / 1.9\end{array}$ & \\
\hline Body 6 & $\begin{array}{l}P R-57-1 \\
P R-61\end{array}$ & $\begin{array}{l}4: 1 \\
2: 1\end{array}$ & & $\begin{array}{l}3.2 \\
5.5\end{array}$ & $\begin{array}{l}1050^{d} \\
1050^{d}\end{array}$ & $\begin{array}{l}350 / 75 / 135 \\
350 / 75 / 120\end{array}$ & $\begin{array}{l}10.1 / 1.1 / 1.9 \\
10.2 / 1.16 / 1.9\end{array}$ & $\cdot$ \\
\hline
\end{tabular}


Part 2. Accelerated Testing in Research Reactors

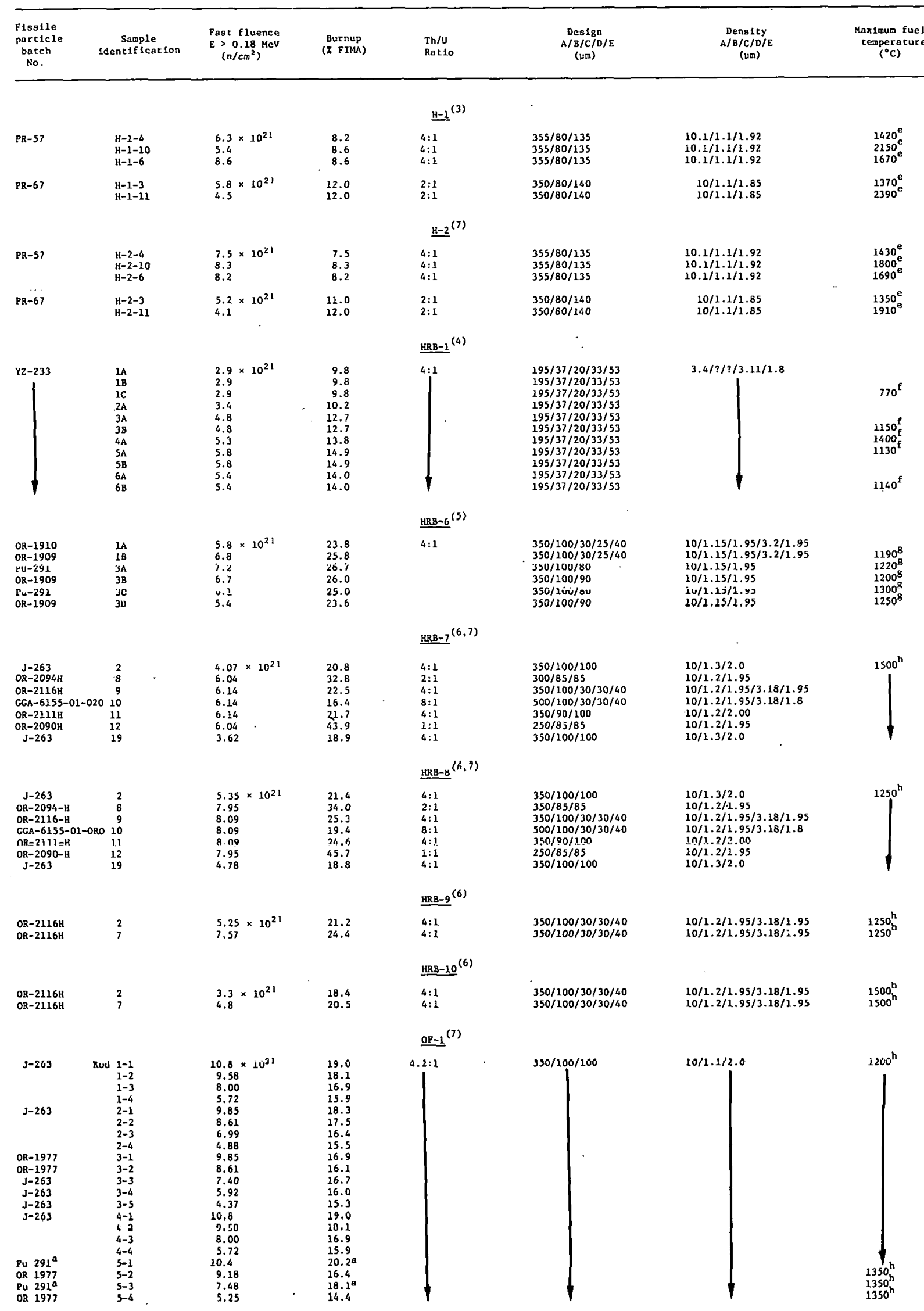


Part 2a. Accelerated Testing in Research Reactors

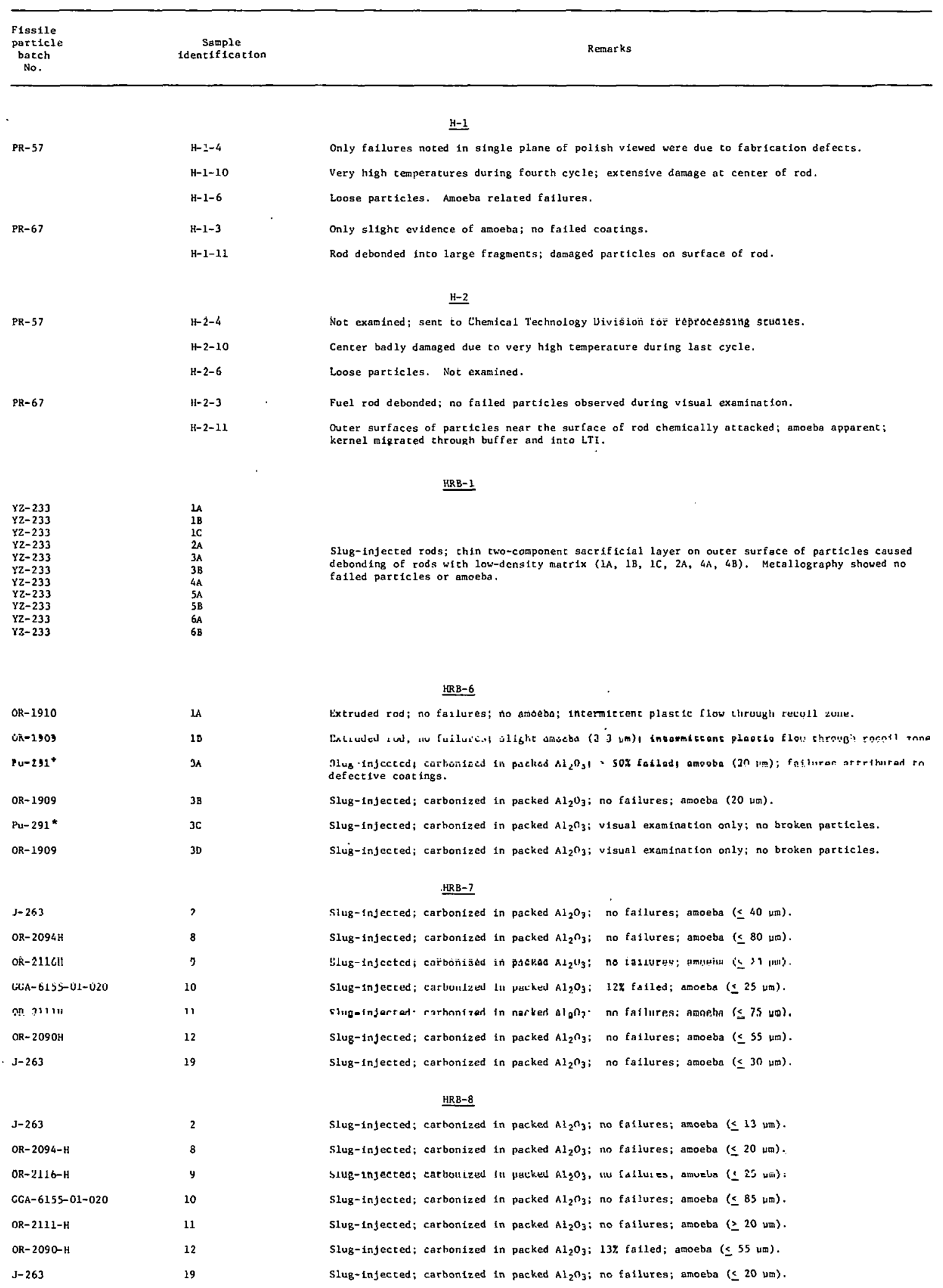


Part 2a. Accelerated Testing in Research Reactors (Continued)

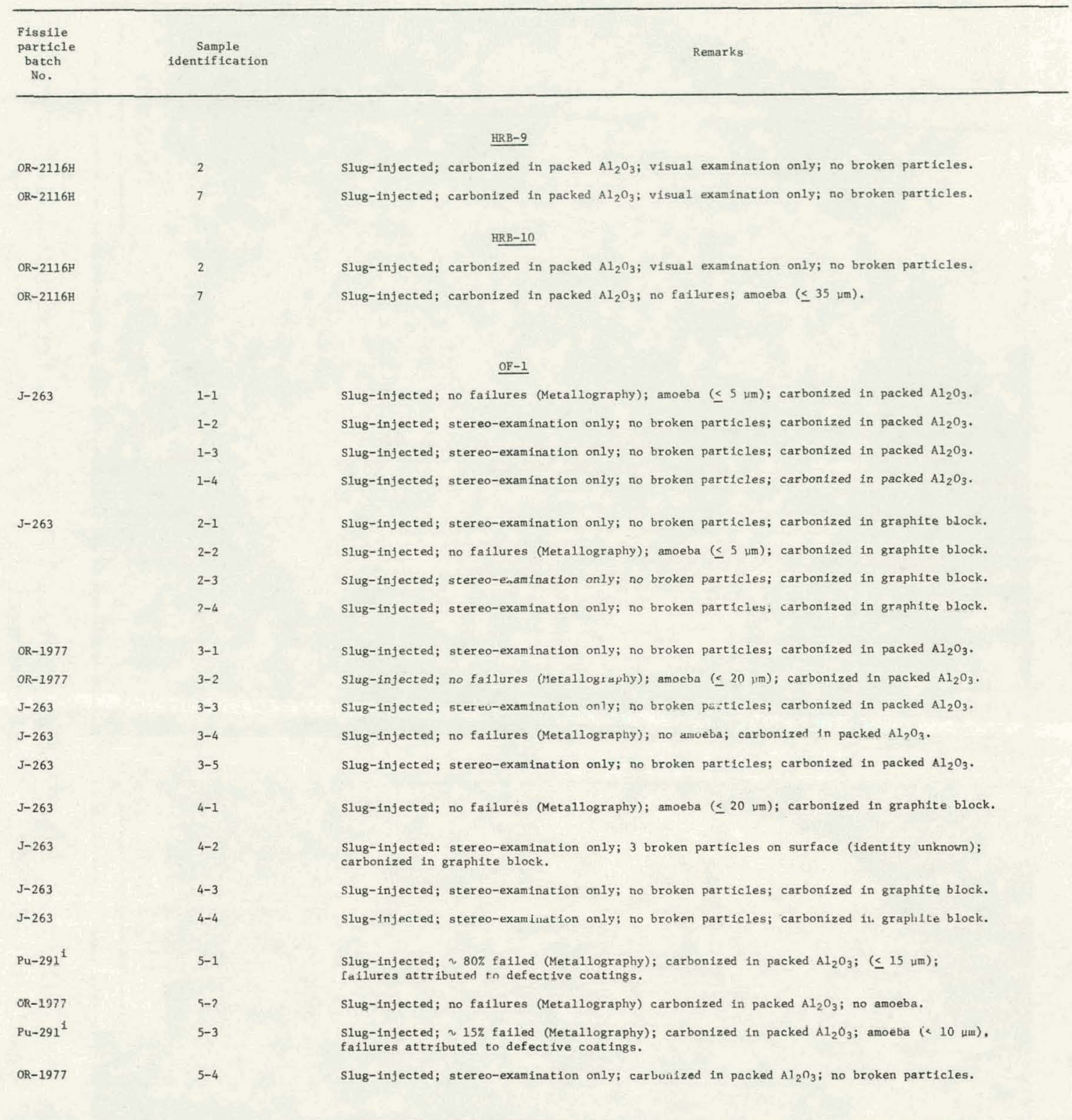

\footnotetext{
$\mathrm{a}_{\mathrm{A}}=$ kernel; $\mathrm{B}=$ buffer; $\mathrm{C}=\mathrm{IPyC} ; \mathrm{D}-\mathrm{SIC} ; \mathrm{E}=$ OPyC.

b Approximately 11,000 to 18,000 fissile particles per rod in RTE fuel rods, depending on particle diameter and density. Forty-eight fuel rods per body.

CThermal analysts to be completed by July 1976.

Maximum fuel temperature in fuel body. These are estimated temperatures based on as-fabricated dimensions, Detailed analyses incorporating irradiation-induced dimensional changes are being performed.

${ }^{e}$ These temperatures represent the maximum calculated for the fuel rod center1ine during the irradiation. This experiment was complicated by a nouintentional inversion of the capsule during the last of four 1rradiation cycles. (See ref. 4 for additional details on fuel operating temperatures.)

${ }^{\mathrm{f}}$ Temperatures for HRB-1 were calculated from readings from thermocouples adjacent to fuel rods. Centerl1ne temperatures were calculated by assuming a fuel rod conductivity of $3.0 \mathrm{Btu} / \mathrm{hr}-\mathrm{ft}-{ }^{\circ} \mathrm{F}$ and a radial gap of $0.004 \mathrm{in}$. Fuel rod $4 \mathrm{~B}$ was composed of a resin binder, and the matrix debonded rather badly during Irradiation. Therefore, the thermal conductivity of the fuel rod may have been as low as 1.5 Btu/hr-ft, ${ }^{\circ} \mathrm{F}$ (a value associated with 100 ra beds
of particle). This change in the bed conductivity increases the calculated fuel centerline temperature from 1230 to $1430^{\circ} \mathrm{C}$.

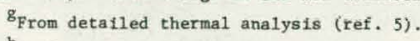

haximum deaign fuel temperatures.

$i_{\text {Fueled with }}{ }^{233} \mathrm{U}$.

References

1E. L. Long, Jr. et a1., Fabrication of ORNL Fuet Irradiated in the Peach Bottom Reactor and Postirradiation Examination of Recycle Test Elemerts 7 and 1, ORNL-TM-44/l (September 1974).

${ }^{2}$ R. P. Morissette and K. P. Steward, Reoycle Teat Elemeni Fruyiwn Devight, Fabriantion, and Asromblu. GA-10109 (Seplember 1971).

${ }^{3}$ R. A. O1stad et al., An Irradiation Test of Candidate HTGR Recycle Fuele in the H-1 and H-8 Capsules, ORNL-TM-4397 (July 1974).

4J. L. Scott et al., An Irradiation Teet of Bonded HTGR Coated Partiole Fuels in an Instmonented Capsule in HFIR, oRNL-TM-3640 (March 1972).

${ }^{5}$ F. J. Homan et al., Irradiation Performance of HTGR Full Rodi in HPIR Experiment HRB-6, ORNL-TM-5011 (December 1975).

${ }^{6}$ K. H. Valentine et a1., Irradiation Performanes of HTGR Fusl Rods in HFIR Experiments in HRB-7 and -8 , in preparation.

${ }^{7}$ HTGR Base Program Progr. Rep. Jan. 1, 1974 to June 30, 1975, ORNL -5108, in preparation.
} 

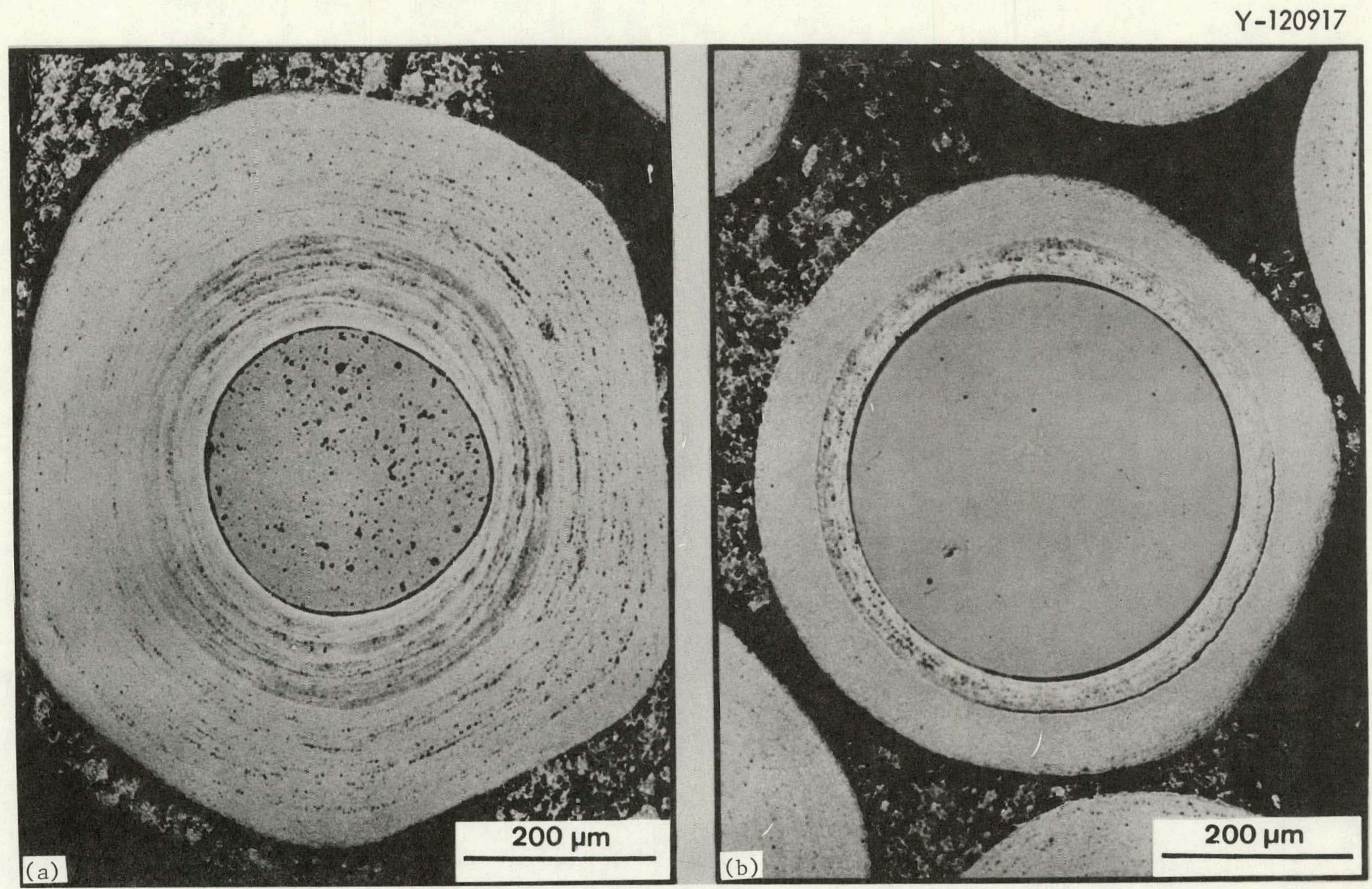

Fig. 4. Relative Thermal Stability of Fissile and Fertile Particles in Irradiation Test H-1-4. Temperature, $1050^{\circ} \mathrm{C}$; thermal gradient, $120{ }^{\circ} \mathrm{C} / \mathrm{cm}$; fast fluence, $6.3 \times 10^{21} \mathrm{n} / \mathrm{cm}^{2}$; time at power, 134 days. (c) ( $4.1 \mathrm{Th}, \mathrm{U}) \mathrm{O}_{2}$ ( $8.2 \%$ FIMA). (b) $\mathrm{ThO}_{2}(0.4 \%$ FIMA). 


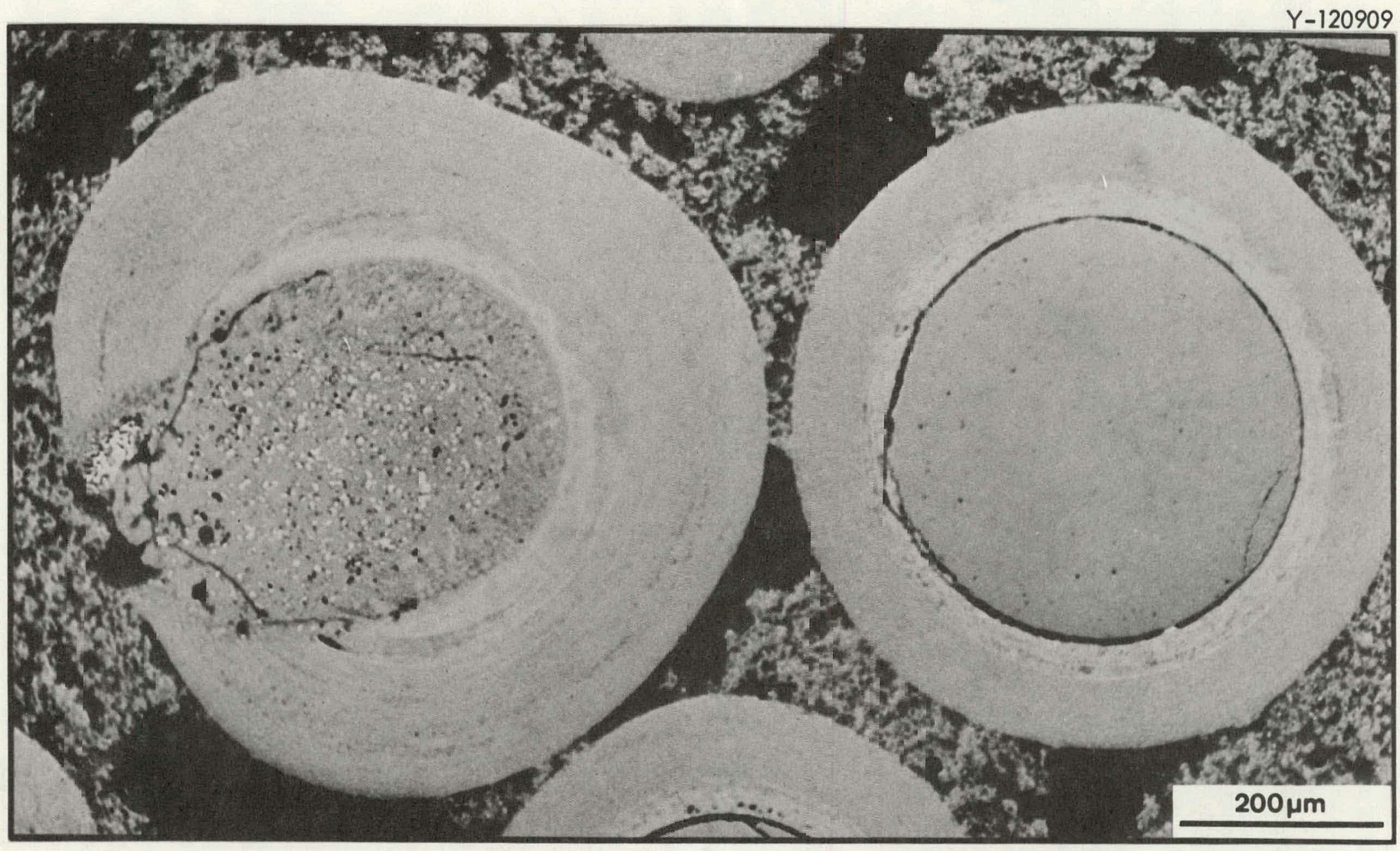

Fig. 5. Relative Thermal Stability of Fissile and Fertile Kernels in Irradiation Test H-2-10. Temperature, $1350^{\circ} \mathrm{C}$; thermal gradient, $1700^{\circ} \mathrm{C} / \mathrm{cm}$; fast flcence, $5 \times 10^{21} \mathrm{n} / \mathrm{cm}^{2}$; time at full power, 37 days. Left: (4 Th, U) $\mathrm{O}_{2}$ ( $8.3 \%$ FTMA). Right: $\mathrm{ThO}_{2}$ ( $0.3 \%$ FIMA). 
These data were treated in similar fashion to the $\mathrm{UO}_{2}$ and $\mathrm{ThO}_{2}$ data shown in Fig. 3. The results are given in Fig. 6. The average curve for $\left(\mathrm{Th}_{0} \cdot{ }_{8} \mathrm{U}_{0} \cdot 2\right) \mathrm{O}_{2}$ falls at the bottom of the cross-hatched critical region in the temperature range $1100-1400^{\circ} \mathrm{C}$. However, when the scatter of the thermal migration data is taken into account and the $90 \%$ confidence intervals are plotted, the upper confidence interval curve extends well into the cross-hatched region. This treatment of the irradiation data for the reference recycle kernel led to the conclusion that this fuel had marginal thermal stability, and an alternate reference fissile fuel was sought. At this stage, additional irradiation testing of other stoichiometries was under way; ${ }^{11}$ however, the results showed little improvement over the reference $(T h / U=4)$ system. A summary of the data from the High Flux Tsntope Reactor (HFIR) removalle beryllium (HRB) -7 capsule ${ }^{11}$ for fuels with Th/U ranging from 0 to 8 is shown in Fig. 7. The thermal stability improves with the addition of thorium to the kernels, but the stability of ( $\left.\mathrm{Th}_{0 .} 8 \mathrm{~g}_{\mathrm{U}} \mathrm{U}_{0.11}\right)_{\mathrm{O}_{2}}$ is about the same as $\left(\mathrm{Th}_{0.8} \mathrm{U}_{0.2}\right)_{2} \mathrm{O}^{9}$

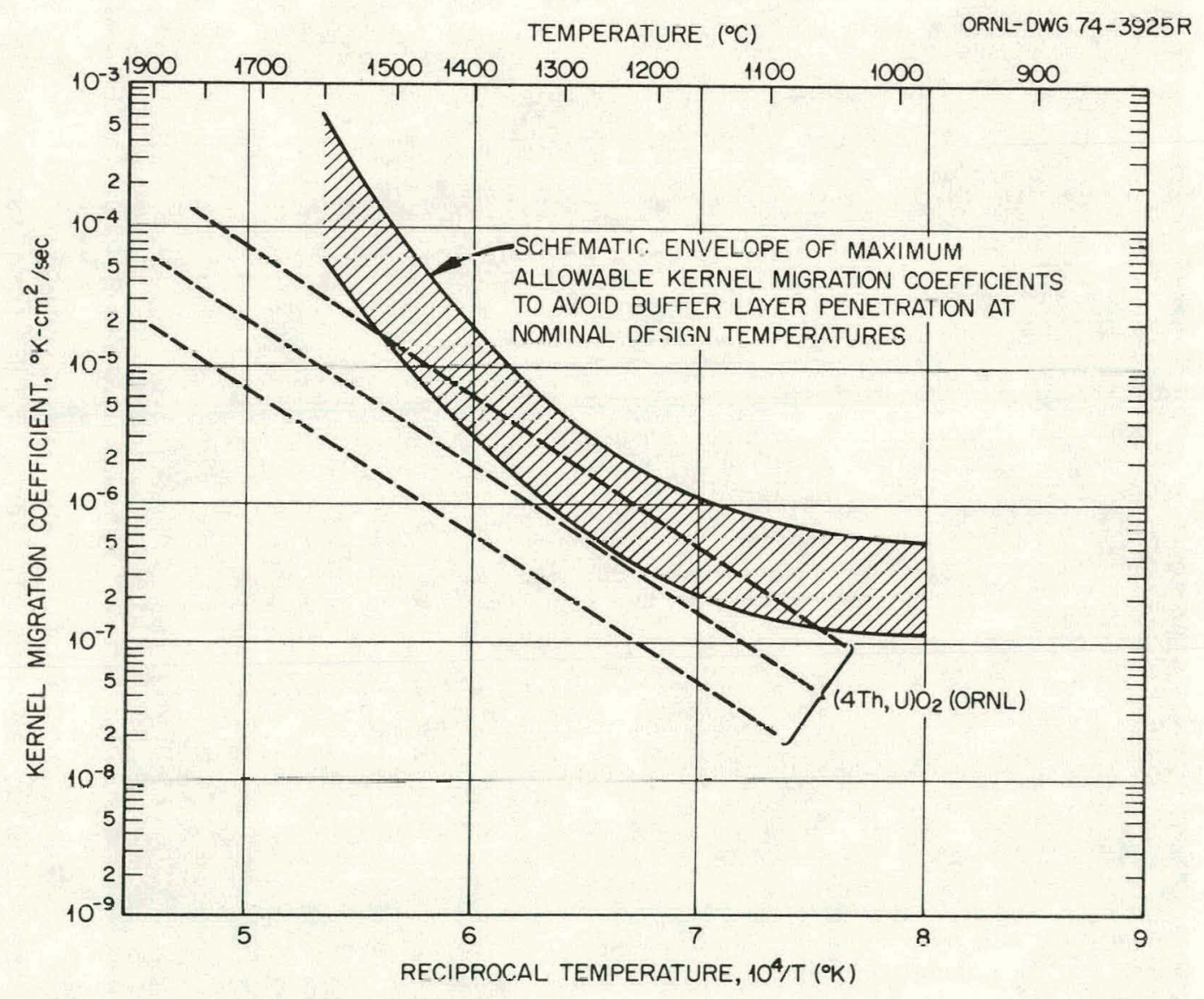

Fig. 6. Average Kernel Migration Coefficients vs Reciprocal Temperature for $(4 \mathrm{Th}, \mathrm{U}) \mathrm{O}_{2}$. 

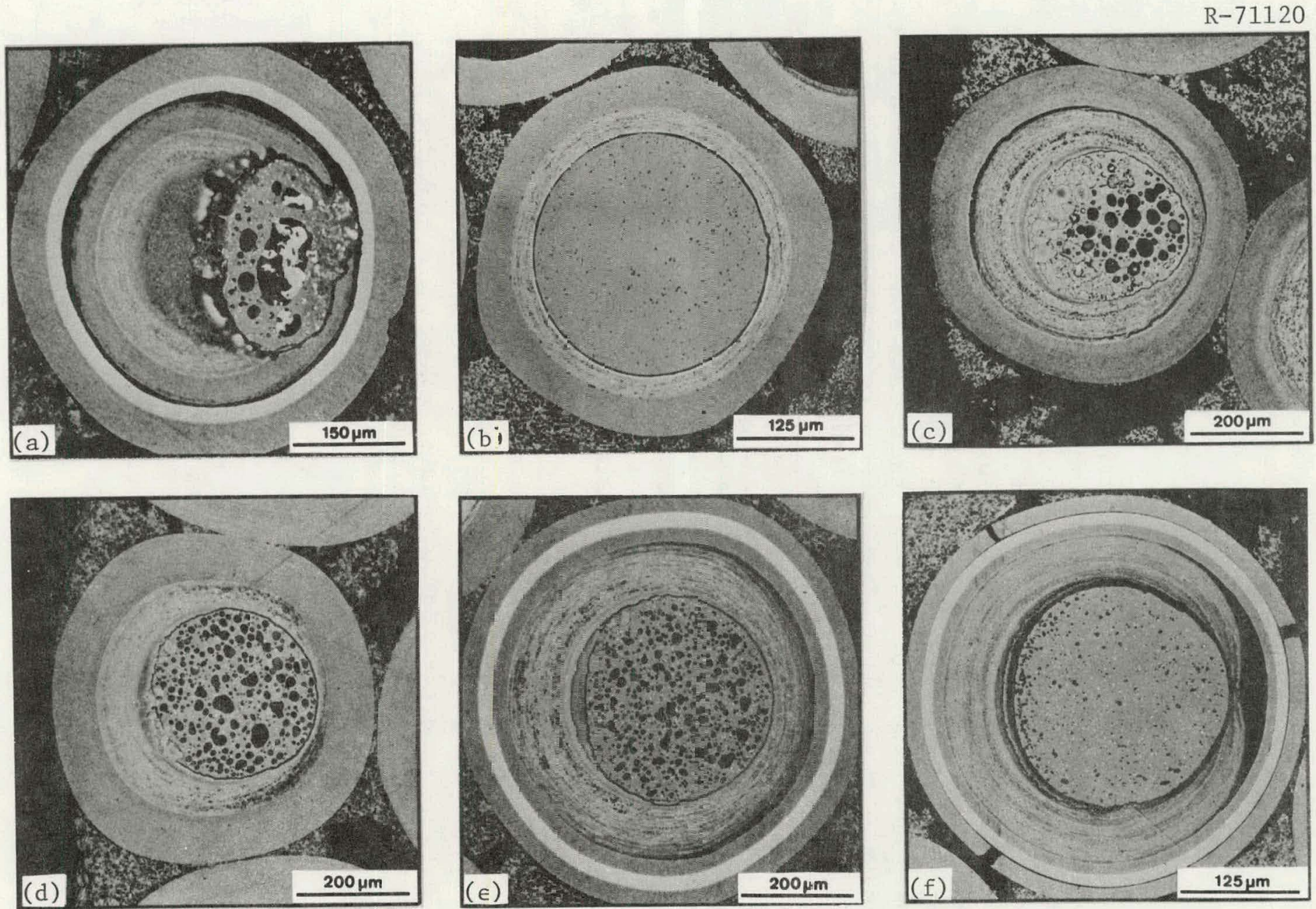

Fig. 7. Relative Thermal Stability of HTGR Candidate Recycle Oxide Fuel Kernels Irradiated in $\mathrm{HRB}-7$. Time-average =emperature, $1200-1220^{\circ} \mathrm{C}$; thermal gradient, $1000-1030^{\circ} \mathrm{C} / \mathrm{cm}$; fast fluence, $6 \times 10^{21} \mathrm{n} / \mathrm{cm}^{2}$. (a) $\mathrm{JO}_{2}(79.8 \% \mathrm{FIM} \sharp)$ (b) $\mathrm{ThO}_{2}$ (8.3\% FIMA). (c) (Th,U) $\mathrm{O}_{2}$ (43.9\% FIMA). (d) $(2 \mathrm{Th}, \mathrm{U}) \mathrm{O}_{2}\left(32.0 \%\right.$ FIMA). (e) $(4 \mathrm{Th}, \mathrm{U}) \mathrm{O}_{2}(22.5 \% \mathrm{FIMA}) \cdot$ (f) (8 Th, U) $\mathrm{O}_{2}$ (16.5\% FIMA). 
The fissile particle selected to replace the dense mixed oxide was an undiluted fissile kernel (containing only a fissile isotope) derived from loading uranium into ion-exchange resins from a weak-acid solution. WAR-derived fuel will be discussed in detail in the next section.

\section{IRRADIATION PERFORMANCE OF WAR FISSILE FUELS}

A summary of the irradiation testing of WAR fuels at ORNL is contained in Table 3. The decision to switch from ( $\left.\mathrm{Th}_{0.8} \mathrm{U}_{0.2}\right)_{2} \mathrm{O}_{2}$ to WAR-derived uranium oxide-carbide as the reference recycle fissile kernel was based on the performance of WAR-dcrived fuel in the HRB-4 and -5 capsiles. ${ }^{12}$ Typical postilıadlation metallography of the WAR fuel irradiated in those capsules is shown in Figs. 8 and 9. Extensive testing of WAR-derived fuels was conducted in capsules HRB-7 and -8 and capsules HRB-9 and $-10,{ }^{11,13}$ In these last four capsules, direct comparisons were made between the performance of WAR fuels of various stoichiometries and dense mixed-oxide fuels, as well as with the reference HTGR fresh fuel (dense $\mathrm{UC}_{2}$ ).

Several observations have been made about WAR fuel performance from this accelerated testing:

1. The porous WAR kernels densify considerably under irradiation, as shown in Fig. 8. The density of the WAR kernels is typically about $3 \mathrm{~g} / \mathrm{cm}^{3}$ before irradiation, compared with densities greater than $10 \mathrm{~g} / \mathrm{cm}^{3}$ for the dense mixed-oxide kernels described earlier. Frequently, the densified WAR kernels remain attacher to one side of the cuating 1 ayers, as shown in Fig. 8. There is no correlation between temperature gradient and the orientation of the densified kernel.

2. There is no evidence of amoeba in WAR kernels containing more than $15 \%$ carbide - with the balance oxide. However, $\mathrm{WAR} \mathrm{UO}_{2}$ kernels have been observed to migrate up the temperature gradient, like the dense oxide kernels described earlier. This can be seen in Fig. 10 in the upper left. Figure 10 summarizes the results of all WAR stoichiometries tested to date. These will be discussed in more detail later in the report.

3. Pliase segregation is associated with WAR kernels after irradiation and is more extensive in the lower conversion levels.* Segregation is shown in the lower left portion of Fig. 10 and in Figs. 11 and 12, which will be described in more detail later.

*Conversion is from $\mathrm{UO}_{2}$ to $\mathrm{UC}_{2}$. In the WAR uranium oxide-carbide kernel, the percent conversion refers to the proportion of $\mathrm{UC}_{2}$ in the two-phase system; $0 \%$ conversion is $\mathrm{UO}_{2}, 15 \%$ conversion is $85 \% \mathrm{UO}_{2}$ and $15 \% \mathrm{UC}_{2}$, etc. 
Table 3. Summary of ORNL Experimental Data on Irradiated Weak-Acid Resin-Derived Recycle Fuel

\begin{tabular}{|c|c|c|c|c|c|c|c|c|c|}
\hline $\begin{array}{l}\text { Fissile } \\
\text { particle } \\
\text { batch } \\
\text { No. }\end{array}$ & $\begin{array}{l}\text { Number of } \\
\text { particles } \\
\text { irradiated }\end{array}$ & $\begin{array}{c}\text { Sample } \\
\text { identification }\end{array}$ & $\begin{array}{l}\text { Fast fluence } \\
\mathrm{E}>0.18 \mathrm{MeV} \\
\left(\mathrm{n} / \mathrm{cm}^{2}\right)\end{array}$ & $\begin{array}{l}\text { Burnup } \\
\text { (\% FIMA) }\end{array}$ & Resin & $\begin{array}{l}\text { Conversion }{ }^{\mathrm{a}} \\
\text { (\%) }\end{array}$ & $\begin{array}{c}\operatorname{Design}^{\mathrm{b}} \\
\mathrm{A} / \mathrm{B} / \mathrm{C} / \mathrm{D} / \mathrm{E} \\
(\mathrm{um})\end{array}$ & $\begin{array}{c}\text { Density }{ }^{\mathrm{b}} \\
\mathrm{A} / \mathrm{B} / \mathrm{C} / \mathrm{D} / \mathrm{E} \\
\left(\mathrm{g} / \mathrm{cm}^{3}\right)\end{array}$ & $\begin{array}{c}\text { Maximum fue } 1^{\mathrm{C}} \\
\text { temperature } \\
\left({ }^{\circ} \mathrm{C}\right)\end{array}$ \\
\hline & \multicolumn{9}{|c|}{$\underline{\mathrm{HRB}-5}^{(1)}$} \\
\hline $52 \mathrm{~A}$ & $\begin{array}{l}5750 \\
5840 \\
5900 \\
2950 \\
2950 \\
2950 \\
2950\end{array}$ & $\begin{array}{l}1 \mathrm{~A} \\
1 \mathrm{~B} \\
1 \mathrm{C} \\
3 \mathrm{~A} \\
3 \mathrm{~B} \\
3 \mathrm{C} \\
3 \mathrm{D}\end{array}$ & $\begin{array}{l}3.4 \times 10^{21} \\
4.0 \\
4.5 \\
4.2 \\
3.9 \\
3.6 \\
3.2\end{array}$ & $\begin{array}{l}13 \\
14 \\
16 \\
15 \\
14 \\
13 \\
12\end{array}$ & IRC- -72 & 95 & $350 / 40 / 30 / 30 / 30$ & $6 / 1.1 / 1.9 / 3.18 / 1.85$ & 1250 \\
\hline & & & & & & ${\mathrm{IRB}-4^{(1)}}^{(1)}$ & & & \\
\hline \multirow[t]{2}{*}{$52 \mathrm{~A}$} & $\begin{array}{l}5750 \\
5840 \\
5900 \\
2950 \\
2950 \\
2950 \\
2950\end{array}$ & $\begin{array}{l}1 \mathrm{~A} \\
1 \mathrm{~B} \\
1 \mathrm{C} \\
3 \mathrm{~A} \\
3 \mathrm{~B} \\
3 \mathrm{C} \\
3 \mathrm{D}\end{array}$ & $\begin{array}{r}7.8 \\
9.2 \\
10.3 \\
9.6 \\
8.9 \\
8.1 \\
7.2\end{array}$ & $\begin{array}{l}24 \\
27 \\
29 \\
27 \\
25 \\
23 \\
20\end{array}$ & IRC-72 & 95 & $350 / 40 / 30 / 30 / 30$ & $\left.\right|^{6 / 1.1 / 1.9 / 3.18 / 1.85}$ & 1250 \\
\hline & \multicolumn{9}{|c|}{$\underline{\mathrm{HRB}-6}^{(2)}$} \\
\hline $\begin{array}{l}\text { Pu-295B } \\
\text { Pu-296 }\end{array}$ & $\begin{array}{l}28 \\
25\end{array}$ & $\begin{array}{l}1 \mathrm{C4} \\
1 \mathrm{C} 5\end{array}$ & $\begin{array}{l}7.7 \\
7.7\end{array}$ & $\begin{array}{l}84 \\
84\end{array}$ & $\begin{array}{l}\text { IRC-72 } \\
\text { IRC-72 }\end{array}$ & $\stackrel{?}{95}$ & $\begin{array}{l}400 / 35 / 25 / 30 / 25 \\
400 / 35 / 25 / 30 / 25\end{array}$ & $\begin{array}{l}3.2 / 1.2 / 1.95 / 3.18 / 1.95 \\
3.8 / 1.2 / 1.95 / 3.18 / 1.95\end{array}$ & 1250 \\
\hline Pu -297 & 22 & $\begin{array}{l}1 \mathrm{C5} \\
1 \mathrm{C6}\end{array}$ & $\begin{array}{l}7.7 \\
7.7\end{array}$ & $\begin{array}{l}84 \\
84\end{array}$ & IRC-72 & 95 & $400 / 35 / 25 / 30 / 25$ & $\begin{array}{l}3,8 / 1.2 / 1.95 / 3.18 / 1.95 \\
3.7 / 1.2 / 1.95 / 3.18 / 1.95\end{array}$ & 1 \\
\hline \multicolumn{10}{|c|}{$\underline{\mathrm{HRB}-7}^{(3)}$} \\
\hline $\begin{array}{l}\text { OR-2115H } \\
\text { OR-2121H }\end{array}$ & $\begin{array}{l}402 \\
407\end{array}$ & $\begin{array}{l}13 \\
14\end{array}$ & 5.9 & 80.0 & IRC-72 & 14 & $400 / 40 / 30 / 30 / 40$ & $3.2 / 1.2 / 1.95 / 3.18 / 1.95$ & 1500 \\
\hline $\begin{array}{l}\mathrm{OR}-2121 \mathrm{H} \\
\mathrm{OR}-2115 \mathrm{H}\end{array}$ & $\begin{array}{l}401 \\
476\end{array}$ & $\begin{array}{l}14 \\
15\end{array}$ & $\begin{array}{l}5.6 \\
5.3\end{array}$ & $\begin{array}{l}79.9 \\
79.9\end{array}$ & $\begin{array}{l}\text { IRC-72 } \\
\text { IRC-72 }\end{array}$ & $\begin{array}{l}92 \\
14\end{array}$ & $\begin{array}{l}300 / 75 / 30 / 30 / 40 \\
400 / 40 / 100\end{array}$ & $\begin{array}{l}5.3 / 1.2 / 1.95 / 3.18 / 1.95 \\
3.2 / 1.2 / 1.95\end{array}$ & \\
\hline $\mathrm{OR}-2121 \mathrm{H}$ & 511 & 16 & 4.9 & 79.9 & IRC-72 & 92 & $\begin{array}{l}400 / 40 / 100 \\
300 / 75 / 85\end{array}$ & $5.3 / 1.2 / 1.95$ & $\varphi$ \\
\hline \multicolumn{10}{|c|}{$\underline{\mathrm{HRB}-8}^{(3)}$} \\
\hline $\begin{array}{l}\mathrm{OR}-2121 \mathrm{H} \\
\mathrm{OR}-2115 \mathrm{H}\end{array}$ & 402 & 13 & 7.7 & 80.4 & IRC-72 & 14 & $\begin{array}{l}400 / 40 / 30 / 30 / 40 \\
300 / 75 / 30 / 30 / 40\end{array}$ & $\begin{array}{l}3.2 / 1.2 / 1.95 / 3.18 / 1.95 \\
5.3 / 1.2 / 1.95 / 3.18 / 1.95\end{array}$ & 1250 \\
\hline $\mathrm{OR}-2115 \mathrm{H}$ & $\begin{array}{l}407 \\
476\end{array}$ & $\begin{array}{l}14 \\
15\end{array}$ & $\begin{array}{l}7.4 \\
6.9\end{array}$ & $\begin{array}{l}80.3 \\
80.3\end{array}$ & $\begin{array}{l}\text { IRC-72 } \\
\text { IRC-72 }\end{array}$ & $\begin{array}{l}92 \\
14\end{array}$ & $\begin{array}{l}300 / 75 / 30 / 30 / 40 \\
400 / 40 / 100\end{array}$ & $\begin{array}{l}5.3 / 1.2 / 1.95 / 3.18 / 1.95 \\
3.2 / 1.2 / 1.95\end{array}$ & \\
\hline $\mathrm{OR}-2115 \mathrm{H}$ & 511 & 16 & $\begin{array}{l}6.9 \\
6.4\end{array}$ & $\begin{array}{l}80.3 \\
80.3\end{array}$ & $\begin{array}{l}\text { IRC-72 } \\
\text { IRC-72 }\end{array}$ & $\begin{array}{l}14 \\
92\end{array}$ & $300 / 75 / 85$ & $5.3 / 1.2 / 1.95$ & 1 \\
\hline \multicolumn{10}{|c|}{$\underline{\mathrm{HRB}-9}^{(4)}$} \\
\hline $\mathrm{OR}-2208 \mathrm{H}$ & 593 & 1 & 4.7 & 79.1 & IRC -72 & 100 & $360 / 50 / 30 / 30 / 40$ & $3.0 / 1.2 / 1.95 / 3.18 / 1.95$ & 1250 \\
\hline $\begin{array}{l}\mathrm{OR}-2121 \mathrm{H} \\
\mathrm{OR}-2218 \mathrm{H}\end{array}$ & 433 & 3 & 5.8 & 79.5 & & 100 & $300 / 75 / 30 / 30 / 40$ & $5.3 / 1.2 / 1.95 / 3.18 / 1.95$ & \\
\hline $\begin{array}{l}\mathrm{OR}-2218 \mathrm{H} \\
\mathrm{OR}-2208 \mathrm{H}\end{array}$ & $\begin{array}{l}486 \\
490\end{array}$ & 4 & 6.3 & 79.4 & & 0 & $360 / 50 / 30 / 30 / 40$ & $3.7 / 1.2 / 1.95 / 3.18 / 2.00$ & \\
\hline $\begin{array}{l}\mathrm{OR}-2208 \mathrm{H} \\
\mathrm{OR}-2115 \mathrm{H}\end{array}$ & $\begin{array}{l}490 \\
373\end{array}$ & $\begin{array}{l}5 \\
8\end{array}$ & 6.8 & 79.9 & & 100 & $360 / 50 / 30 / 30 / 40$ & $3.0 / 1.2 / 1.95 / 3.18 / 1.95$ & \\
\hline $\mathrm{OR}-2207 \mathrm{H}$ & $\begin{array}{l}373 \\
486\end{array}$ & $\begin{array}{l}8 \\
9\end{array}$ & 7.8 & 80.1 & & $\begin{array}{l}15 \\
75\end{array}$ & $\begin{array}{l}400 / 40 / 30 / 30 / 40 \\
360 / 50 / 30 / 30 / 40\end{array}$ & & \\
\hline $\mathrm{OR}-2207 \mathrm{H}$ & 486 & $\begin{array}{l}9 \\
10\end{array}$ & & $\begin{array}{l}80.1 \\
80.1\end{array}$ & & 75 & $\begin{array}{l}360 / 50 / 30 / 30 / 40 \\
360 / 50 / 30 / 30 / 40\end{array}$ & $3.0 / 1.2 / 1.95 / 3.18 / 1.95$ & \\
\hline OR-22U8H & 469 & 11 & 7.9 & 80.1 & & 100 & $360 / 50 / 30 / 30 / 40$ & $3.0 / 1.2 / 1.95 / 3.13 / 1.95$ & \\
\hline $\mathrm{OR}-2218 \mathrm{H}$ & 423 & 12 & 7.8 & 80.1 & & 0 & $360 / 50 / 30 / 30 / 40$ & $3.7 / 1.2 / 1.95 / 3.18 / 2.00$ & \\
\hline OR-2211H & 520 & 13 & 7.6 & 80.0 & & 50 & $360 / 50 / 30 / 30 / 40$ & $3.1 / 1.2 / 1.95 / 3.18 / 2.00$ & \\
\hline $\mathrm{OR}-2121 \mathrm{H}$ & 398 & 14 & 7.2 & 79.9 & & 100 & $300 / 75 / 30 / 30 / 40$ & $5.3 / 1.2 / 1.95 / 3.18 / 1.95$ & \\
\hline $\mathrm{OR}-2207 \mathrm{H}$ & 510 & 15 & 6.8 & 79.9 & & 75 & $360 / 50 / 30 / 30 / 40$ & $3.0 / 1.2 / 1.95 / 3.18 / 1.95$ & \\
\hline OR-2115I & 427 & 16 & 6.3 & 80.0 & & 15 & $400 / 40 / 30 / 30 / 40$ & $3.2 / 1.2 / 1.95 / 3.18 / 1.95$ & \\
\hline $\mathrm{OR}-2211 \mathrm{H}$ & 566 & 17 & 5.8 & 79.5 & & 50 & $360 / 50 / 30 / 30 / 40$ & $3.1 / 1.2 / 1.95 / 3.18 / 2.00$ & \\
\hline $\mathrm{OR}-2219 \mathrm{H}$ & 584 & 18 & 5.3 & 79.3 & & $\mathrm{NA}$ & $360 / 50 / 30 / 30 / 40$ & $3.0 / 1.2 / 1.95 / 3.18 / 2.00$ & \\
\hline $\mathrm{OR}-2207 \mathrm{H}$ & 616 & 19 & 4.7 & 79.1 & & 75 & $36 n / 5 n / 3 n / 3 n / 4 n$ & $3.0 / 1.2 / 1.95 / 3.18 / 1,95$ & 1 \\
\hline $\mathrm{OR}-2219 \mathrm{H}$ & 484 & 6 & 7.2 & 80.0 & & NA & $360 / 50 / 30 / 30 / 40$ & $3.0 / 1.2 / 1.95 / 3.18 / 2.00$ & \\
\hline \multicolumn{10}{|c|}{$\underline{\mathrm{HRB}-10}^{(4)}$} \\
\hline $\mathrm{OR}-2208 \mathrm{H}$ & 593 & 1 & 3.0 & 77.3 & IRC-72 & 100 & $360 / 50 / 30 / 30 / 40$ & $3.0 / 1.2 / 1.95 / 3.18 / 1.95$ & 1500 \\
\hline OR-2121H & 433 & 3 & 3.7 & 78.5 & & 100 & $300 / 75 / 30 / 30 / 40$ & $5.3 / 1.2 / 1.95 / 3.18 / 1.95$ & \\
\hline $\mathrm{OR}-2218 \mathrm{H}$ & 486 & 4 & 4.0 & 79.2 & & 0 & $360 / 50 / 30 / 30 / 40$ & $3.7 / 1.2 / 1.95 / 3.18 / 2.00$ & \\
\hline $\mathrm{OR}-2208 \mathrm{H}$ & 490 & 5 & 4.3 & 79.2 & & 100 & $360 / 50 / 30 / 30 / 40$ & $3.0 / 1.2 / 1.95 / 3.18 / 1.95$ & \\
\hline $\mathrm{OR}-2115 \mathrm{H}$ & 373 & 8 & 4.9 & 79.5 & & 15 & $400 / 40 / 30 / 30 / 40$ & $3.2 / 1,2 / 1.95 / 3.18 / 1.95$ & \\
\hline OR-2207H & 486 & 9 & 5.0 & 79.6 & & 75 & $360 / 50 / 30 / 30 / 40$ & $3.0 / 1.2 / 1.95 / 3.18 / 1.95$ & \\
\hline $\mathrm{OR}-2207 \mathrm{H}$ & 486 & 10 & 5.0 & 79.6 & & 75 & $360 / 50 / 30 / 30 / 40$ & $3.0 / 1.2 / 1.95 / 3.18 / 1.95$ & \\
\hline $\mathrm{OR}-2208 \mathrm{H}$ & 469 & 11 & 5.0 & 79.6 & & 100 & $360 / 50 / 30 / 30 / 40$ & $3.0 / 1.2 / 1.95 / 3.18 / 1.95$ & \\
\hline $\mathrm{OR}-2218 \mathrm{H}$ & 423 & 12 & 4.9 & 79.5 & & 0 & $360 / 50 / 30 / 30 / 40$ & $3.7 / 1.2 / 1.95 / 3.18 / 2.00$ & \\
\hline $\mathrm{OR}-2211 \mathrm{H}$ & 520 & 13 & 4.8 & 79.4 & & 50 & $360 / 50 / 30 / 30 / 40$ & $3.1 / 1.2 / 1.95 / 3.18 / 2.00$ & \\
\hline $\mathrm{OR}-2121 \mathrm{H}$ & 398 & 14 & 4.6 & 79.3 & & 100 & $300 / 75 / 30 / 30 / 40$ & $5.3 / 1.2 / 1.95 / 3.18 / 1.95$ & \\
\hline OR-2207H & 510 & 15 & 4.3 & 79.2 & & 75 & $360 / 50 / 30 / 30 / 40$ & $3.0 / 1.2 / 1.95 / 3.18 / 1.95$ & \\
\hline OR-2115I & 427 & 16 & 4,0 & 79.2 & & 15 & $400 / 40 / 30 / 30 / 40$ & $3.2 / 1.2 / 1.95 / 3.18 / 1.95$ & \\
\hline $\mathrm{OR}-2211 \mathrm{H}$ & 366 & 17 & 3.7 & 78.5 & & 50 & $360 / 50 / 30 / 30 / 40$ & $3.1 / 1.2 / 1.95 / 3.18 / 2.00$ & \\
\hline $\mathrm{OR}-2219 \mathrm{H}$ & 584 & 18 & 3.3 & 78.0 & & NA & $360 / 50 / 30 / 30 / 40$ & $3.0 / 1.2 / 1.95 / 3.18 / 2.00$ & \\
\hline $\mathrm{OR}-2207 \mathrm{H}$ & 616 & 19 & 3.0 & 77.3 & & 75 & $360 / 50 / 30 / 30 / 40$ & $3.0 / 1.2 / 1.95 / 3.18 / 1.95$ & \\
\hline $\mathrm{OR}-2219 \mathrm{H}$ & 484 & 6 & 4.6 & 79.3 & & NA & $360 / 50 / 30 / 30 / 40$ & $3.0 / 1.2 / 1.95 / 3.18 / 2.00$ & \\
\hline \multicolumn{10}{|c|}{$\underline{\mathrm{OP}-2}^{\left(\mathrm{Ce} 112^{2}\right)^{(4)}}$} \\
\hline$A-611^{d}$ & 4450 & $A-1-1$ & e & $f$ & IRC-72 & 15 & $360 / 50 / 35 / 30 / 35$ & $3.0 / 1.1 / 1.7 />3.18 / 1.7$ & 1150 \\
\hline$A-601^{d}$ & 4440 & $\begin{array}{r}A-1-1 \\
-2\end{array}$ & e & f & & 75 & $360 / 50 / 35 / 30 / 35$ & $3.0 / 1.1 / 1.7 />3.18 / 1.2$ & 1150 \\
\hline $\mathrm{A}-611^{\mathrm{d}}$ & 2530 & -3 & e & $f$ & & 15 & $360 / 50 / 35 / 30 / 35$ & $3.0 / 1.1 / 1.7 />3.18 / 1.7$ & \\
\hline A-611 d & 2530 & -4 & e & f & & 15 & $360 / 50 / 35 / 30 / 35$ & $3.0 / 1.1 / 1.7 />3.18 / 1.7$ & \\
\hline$A-601^{d}$ & 1980 & -5 & e & f & & 75 & $360 / 50 / 35 / 30 / 35$ & $3.0 / 1.1 / 1.7 />3.18 / 1.7$ & \\
\hline$A-601^{d}$ & 1980 & -6 & e & $\mathrm{f}$ & & 75 & $360 / 50 / 35 / 30 / 35$ & $3.0 / 1.1 / 1.7 />3.18 / 1.7$ & \\
\hline$A-611^{d}$ & 4450 & $A-2-1$ & e & f & & 1.5 & $360 / 50 / 35 / 30 / 35$ & $3.0 / 1.1 / 1.7 / 33.18 / 1.7$ & \\
\hline$A-611^{d}$ & 4450 & -2 & e & f & & 13 & $360 / 50 / 35 / 30 / 35$ & $3.0 / 1.1 / 17 / 318 / 17$ & \\
\hline$A-601^{d}$ & 2520 & -3 & e & $\mathrm{f}$ & & 75 & $360 / 50 / 35 / 50 / 35$ & $3.0 / 1.1 / 1.7 />3.18 / 1.7$ & \\
\hline$A-601^{d}$ & 2520 & -4 & e & $\mathrm{f}$ & & 75 & $360 / 50 / 35 / 50 / 35$ & $3.0 / 1.1 / 1.7 />3.18 / 1.7$ & \\
\hline$A-611^{d}$ & 1980 & -5 & e & $\mathrm{f}$ & & 15 & $360 / 50 / 35 / 50 / 35$ & $3.0 / 1.1 / 1.7 />3.18 / 1.7$ & 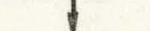 \\
\hline$A-601^{d}$ & 1980 & -6 & e & f & & 75 & $360 / 50 / 35 / 50 / 35$ & $3.0 / 1.1 / 1.7 />3.18 / 1.7$ & \\
\hline
\end{tabular}


Table 3. (Continued)

\begin{tabular}{|c|c|c|c|c|c|c|c|c|c|}
\hline $\begin{array}{l}\text { Fissile } \\
\text { particle } \\
\text { batch } \\
\text { No. }\end{array}$ & $\begin{array}{l}\text { Number of } \\
\text { particles } \\
\text { irradiated }\end{array}$ & $\begin{array}{c}\text { Sample } \\
\text { identification }\end{array}$ & $\begin{array}{l}\text { Fast fluence } \\
\mathrm{E}>0.18 \mathrm{MeV} \\
\left(\mathrm{n} / \mathrm{cm}^{2}\right)\end{array}$ & $\begin{array}{l}\text { Burnup } \\
(\% \text { FIMA })\end{array}$ & Resin & $\begin{array}{l}\text { Conversion }{ }^{a} \\
(\%)\end{array}$ & $\begin{array}{c}\text { Design } \\
\mathrm{A} / \mathrm{B} / \mathrm{C} / \mathrm{N} / \mathrm{E} \\
(\mu \mathrm{m})\end{array}$ & $\begin{array}{c}\text { Density } \\
\mathrm{A} / \mathrm{B} / \mathrm{C} / \mathrm{D} / \mathrm{E} \\
\left(\mathrm{g} / \mathrm{cm}^{3}\right)\end{array}$ & $\begin{array}{c}\text { Maximum fuel }{ }^{c} \\
\text { temperature } \\
\left({ }^{\circ} \mathrm{C}\right)\end{array}$ \\
\hline OR-2329H & 1920 & $A-3-2$ & e & f & \multirow[t]{7}{*}{ IRC-72 } & 0 & $375 / 20 / 35 / 30 / 35$ & $3.2 /<1.3 / 1.95 />3.18 / 2.0$ & \multirow{16}{*}{1150} \\
\hline OR-2329H & 1920 & A-3-4 & e & $\mathrm{f}$ & & 0 & $375 / 20 / 35 / 30 / 35$ & $3.2 /<1.3 / 1.95 />3.18 / 2.0$ & \\
\hline $\begin{array}{l}\text { OR-2322H } \\
\text { OR-2211H }\end{array}$ & $\begin{array}{l}1080 \\
1100\end{array}$ & $\begin{array}{l}A-3-5 \\
A-3-6\end{array}$ & e & $\mathrm{E}$ & & 15 & $360 / 50 / 35 / 30 / 35$ & $3.1 /<1.3 / 1.95 />3.18 / 2.0$ & \\
\hline OR-2208H & $\begin{array}{l}1100 \\
1040\end{array}$ & $\begin{array}{l}A-3-6 \\
A-3-7\end{array}$ & e & f & & 50 & $375 / 50 / 35 / 30 / 35$ & $3.2 /<1.3 / 1.95 />3.18 / 2.0$ & \\
\hline $\mathrm{OR}-2322 \mathrm{H}$ & $\begin{array}{r}1040 \\
850\end{array}$ & $\begin{array}{l}A-3-1 \\
A-3-9\end{array}$ & $\begin{array}{l}\mathrm{e} \\
\mathrm{e}\end{array}$ & $\begin{array}{l}t \\
f\end{array}$ & & $\begin{array}{r}100 \\
15\end{array}$ & $\begin{array}{l}360 / 50 / 35 / 30 / 35 \\
375 / 50 / 35 / 30 / 35\end{array}$ & $\begin{array}{l}3.0 / 1.3 / 1.95 />3.18 / 2.0 \\
3.2<1.3 / 1.95 / 3.18 / 2.0\end{array}$ & \\
\hline OR-2329H & 850 & $A-3-10$ & e & f & & 0 & $375 / 20 / 35 / 30 / 35$ & $3.2 k 1.3 / 1.95 / 3.18 / 2.0$ & \\
\hline OR-2208H & 820 & $A-3-11$ & e & f & & 100 & $360 / 50 / 35 / 30 / 35$ & $3.0 / 1.3 / 1.95 / 3.18 / 2.0$ & \\
\hline $\mathrm{OR}-2121 \mathrm{H}$ & 1520 & $A-4-1$ & e & f & \multirow{9}{*}{ IRC-72 } & 100 & $320 / 75 / 35 / 30 / 35$ & $5.0 /<1.3 / 1.95 />3.18 / 2.0$ & \\
\hline OR-2332H & 1940 & $A-4-2$ & e & f & & 0 & $375 / 50 / 35 / 30 / 35$ & $3.2 /<1.3 / 1.95 />3.18 / 2.0$ & \\
\hline OR-2121H & 1520 & $A-4-3$ & e & $\mathrm{f}$ & & 100 & $320 / 75 / 35 / 30 / 35$ & $5.0 /<1.3 / 1.95 />3.18 / 2.0$ & \\
\hline $\mathrm{OR}-2332 \mathrm{H}$ & 1940 & $A-4-4$ & e & $\mathrm{f}$ & & 0 & $375 / 50 / 35 / 30 / 35$ & $3.2 /<1.3 / 1.95 />3.18 / 2.0$ & \\
\hline $\mathrm{OR}-2218 \mathrm{H}$ & 960 & $A-4-5$ & e & $f$ & & 0 & $360 / 50 / 35 / 3 n / 35$ & $3.7 /<1.3 / 1.95 />3.18 / 2.0$ & \\
\hline $\mathrm{OR}-2320 \mathrm{H}$ & $110 n$ & $A-4=6$ & e & i & & 25 & $375, i 50$ issisuiss & $3.2 /<1.3 / 1.95 />3.18 / 2.0$ & \\
\hline on $22071 \mathrm{I}$ & 1083 & $\begin{array}{l}A-4-1 \\
A-4-9\end{array}$ & e & $\mathrm{f}$ & & 75 & $360 / 50 / 35 / 30 / 35$ & $3.0 /<1.3 / 1.95 />3.18 / 2.0$ & \\
\hline & 750 & $A-4-9$ & e & $f$ & & 0 & $360 / 50 / 35 / 30 / 39$ & $3.1 /<1.3 / 1.95 / 33.18 / 2.0$ & \\
\hline $\begin{array}{l}\mathrm{OR}-2332 \mathrm{H} \\
\mathrm{OR}-2207 \mathrm{H}\end{array}$ & $\begin{array}{l}860 \\
850\end{array}$ & $A=4-1 U$ & $\begin{array}{l}e \\
\text { e }\end{array}$ & $\begin{array}{l}f \\
f\end{array}$ & & $\begin{array}{r}0 \\
75\end{array}$ & $\begin{array}{l}375 / 50 / 35 / 30 / 35 \\
360 / 50 / 35 / 30 / 35\end{array}$ & $\begin{array}{l}3.2 /<1.3 / 1.95 />3.18 / 2.0 \\
3.0 /<1.3 / 1.95 />3.18 / 2.0\end{array}$ & \\
\hline$A-601^{d}$ & 1520 & $B-1-1$ & e & f & \multirow{10}{*}{ IRC-72 } & 75 & \multirow{10}{*}{$360 / 50 / 35 / 30 / 35$} & \multirow{10}{*}{$3.0 / 1.1 / 1.8 / 23.18 / 1.8$} & \multirow{10}{*}{1350} \\
\hline$A-6 n 1^{d}$ & 1520 & $\mathrm{D}-1-2$ & e & $\mathrm{f}$ & & 75 & & & \\
\hline$A-601^{d}$ & 1290 & $B-1-3$ & e & $\mathrm{f}$ & & 75 & & & \\
\hline$A-611^{d}$ & 1290 & $\mathrm{~B}-1-4$ & c & $i$ & & 15 & & & \\
\hline$A-611^{d}$ & 1290 & $B-1-5$ & $=$ & i & & 15 & & & \\
\hline$A-611^{d}$ & 1290 & $\mathrm{~B}-1-6$ & e & f & & 15 & & & \\
\hline $\begin{array}{l}A-601^{d} \\
A-611^{d}\end{array}$ & $\begin{array}{l}1520 \\
1520\end{array}$ & $\begin{array}{l}B-2-1 \\
B-2-2\end{array}$ & $\begin{array}{l}e \\
e\end{array}$ & $\begin{array}{l}f \\
f\end{array}$ & & $\begin{array}{l}75 \\
15\end{array}$ & & & \\
\hline$A-611^{d}$ & 1290 & $B-2-3$ & c & f & & 15 & & & \\
\hline $\begin{array}{l}A-601^{d} \\
A-601^{d}\end{array}$ & $\begin{array}{l}1290 \\
1290\end{array}$ & $\begin{array}{l}\text { B-2-4 } \\
\text { B-2-5 }\end{array}$ & $\begin{array}{l}\text { c } \\
\text { e }\end{array}$ & $\begin{array}{l}f \\
f\end{array}$ & & $\begin{array}{l}75 \\
75\end{array}$ & & & \\
\hline$A-611^{d}$ & 1290 & $B-2-6$ & e & $\mathrm{f}$ & & 15 & & & \\
\hline $\mathrm{OR}-2329 \mathrm{H}$ & 660 & B $-3-2$ & e & $\mathrm{f}$ & \multirow{9}{*}{$\mathrm{IRC}-72$} & 0 & $375 / 20 / 35 / 30 / 35$ & $3.2 /<1.3 / 1.95 />3.18 / 2.0$ & \\
\hline $\mathrm{OR}-2322 \mathrm{H}$ & 650 & $B-3-3$ & e & f & & 15 & $375 / 50 / 35 / 30 / 35$ & $3.2 /<1.3 / 1.95 / 33.18 / 2.0$ & \\
\hline$O R-2211 \mathrm{H}$ & 660 & $R-3-4$ & e & f & & 50 & $360 / 50 / 35 / 30 / 35$ & $3.1 /<1.3 / 1.95 />3.18 / 2.0$ & \\
\hline OR-2208H & 530 & $B-3-5$ & e & $\mathrm{f}$ & & 100 & $360 / 50 / 35 / 30 / 35$ & & \\
\hline OR-2329H & 560 & $B-3-7$ & e & $\mathrm{f}$ & & 0 & $375 / 20 / 35 / 30 / 35$ & $3.2 /<1.3 / 1.95 />3.18 / 2.0$ & \\
\hline $\mathrm{OR}-2322 \mathrm{H}$ & 550 & $B-3-8$ & e & f & & 15 & $375 / 50 / 35 / 30 / 35$ & $3.2 /<1.3 / 1.95 />3.18 / 2.0$ & \\
\hline $\begin{array}{l}\mathrm{OR}-2218 \mathrm{H} \\
\mathrm{OR}-2208 \mathrm{H}\end{array}$ & $\begin{array}{l}490 \\
530\end{array}$ & $\begin{array}{l}B-3-9 \\
B-3-10\end{array}$ & e & f & & 0 & $360 / 50 / 35 / 30 / 35$ & $3.7 /<1.3 / 1.95 />3.18 / 2.0$ & \\
\hline $\mathrm{OR}-2329 \mathrm{H}$ & 560 & $\begin{array}{l}B-3-10 \\
B-3-11\end{array}$ & $\begin{array}{l}\mathrm{e} \\
\mathrm{e}\end{array}$ & $\begin{array}{l}f \\
f\end{array}$ & & $\begin{array}{r}100 \\
0\end{array}$ & $\begin{array}{l}360 / 50 / 35 / 30 / 35 \\
375 / 20 / 35 / 30 / 35\end{array}$ & $\begin{array}{l}3.0 /<1.3 / 1.95 />3.18 .2 .0 \\
3.2 /<1.3 / 1.95 />3.18 / 2,0\end{array}$ & \\
\hline $\mathrm{OR}-2322 \mathrm{H}$ & 550 & $B-3-12$ & e & $f$ & & 15 & $375 / 50 / 35 / 30 / 35$ & $3.2 /<1.3 / 1.95 />3.18 / 2.0$ & \\
\hline OR-2332II & 660 & $B-4-2$ & e & $\mathrm{f}$ & \multirow{9}{*}{ IRC-72 } & 0 & $375 / 50 / 35 / 30 / 35$ & $3.2 /<1.3 / 1.95 />3.18 / 2.0$ & \\
\hline $\mathrm{OR}-2218 \mathrm{H}$ & 580 & $B-4-3$ & e & $\mathrm{f}$ & & 0 & $360 / 50 / 39 / 30 / 39$ & $3 . / /<1.3 / 1.95 />3.18 / 2.0$ & \\
\hline $\mathrm{NR}-232 \mathrm{NE}$ & 660 & $B-4=4$ & e & $f$ & & 25 & $375 / 50 / 35 / 30 / 35$ & $3.2 /<1.3 / 1.95 />3.18 / 2.0$ & \\
\hline OR-2207H & 550 & $B-4-5$ & e & $\mathrm{f}$ & & 75 & $320 / 75 / 35 / 30 / 35$ & $5.0 /<1.3 / 1.95 />3.18 / 2.0$ & \\
\hline $\mathrm{OR}-2121 \mathrm{H}$ & 440 & $B-4-6$ & e & $\mathrm{f}$ & & 100 & $320 / 75 / 35 / 30 / 35$ & $5.0 /<1.3 / 1.95 />3.18 / 2.0$ & \\
\hline$O R-2332 H$ & 560 & $B-4-1$ & e & f & & 0 & $375 / 50 / 35 / 30 / 35$ & $3.2 /<1.3 / 1.95 />3.18 / 2.0$ & \\
\hline OR-2218B & 490 & $B-4-8$ & e & $\mathrm{f}$ & & 0 & $360 / 50 / 35 / 30 / 35$ & $3.7 /<1.3 / 1.95 />3.18 / 2.0$ & \\
\hline $\begin{array}{l}\mathrm{OR}-2.32 \mathrm{OH} \\
\mathrm{OR}-2207 \mathrm{H}\end{array}$ & $\begin{array}{l}560 \\
59 n\end{array}$ & $\begin{array}{l}\text { B- }-4-9 \\
\text { B } 410\end{array}$ & $\begin{array}{l}\text { e } \\
\text { e }\end{array}$ & $\begin{array}{l}f \\
f\end{array}$ & & $\begin{array}{l}25 \\
75\end{array}$ & $\begin{array}{l}375 / 50 / 35 / 30 / 35 \\
320 / 75 / 35 / 30 / 35\end{array}$ & $\begin{array}{l}3.21<1.3 / 1.95 />3.18 / 2.0 \\
5.0 /<1.3 / 1.95 />3.18 / 2.0\end{array}$ & \\
\hline \multirow[t]{2}{*}{ OR 233211} & 360 & $B-4-11$ & e & $\mathrm{f}$ & & 0 & $375 / 50 / 35 / 30 / 35$ & $3.2 /<1.3 / 1.95 />3.18 / 2.0$ & \\
\hline & \multicolumn{8}{|c|}{${\mathrm{OF}-2(\mathrm{Cel1} 1)^{(4)}}^{(4)}$} & \\
\hline$A-601^{d}$ & 2500 & C-1-1 & e & $\mathrm{f}$ & IRC-72 & 75 & $360 / 50 / 35 / 30 / 35$ & $3.1 / 1.1 / 1.8 />3.18 / 1.8$ & \\
\hline$A-601 d$ & 2500 & $\mathrm{C}-1-2$ & e & f & & 75 & & & \\
\hline $\begin{array}{l}A-611^{d} \\
A-601^{d}\end{array}$ & $\begin{array}{l}2970 \\
6790\end{array}$ & $\begin{array}{l}\mathrm{C}-1-3 \\
\mathrm{c}-1-4\end{array}$ & $\begin{array}{l}e \\
e\end{array}$ & $\begin{array}{l}f \\
f\end{array}$ & & $\begin{array}{l}15 \\
75\end{array}$ & & & \\
\hline$A-6158$ & 2440 & $c-2-1$ & e & $\mathrm{f}$ & & 75 & & & \\
\hline$A-601^{d}$ & 2500 & $\mathrm{C}-2-2$ & e & $\mathrm{f}$ & & 75 & & & \\
\hline A- $615^{8}$ & 2900 & $\mathrm{C}-2-3$ & e & f & & 75 & & & \\
\hline$A-615^{8}$ & 6648 & $C-2-4$ & e & f & & 75 & & & \\
\hline$A-611^{d}$ & 2500 & c-3-1 & e & f & & 13 & & & \\
\hline $\begin{array}{l}A 611^{d} \\
A-601^{d}\end{array}$ & $\begin{array}{l}\text { ijuU } \\
2960\end{array}$ & $\begin{array}{l}\mathrm{L}-3-2 \\
\mathrm{C}-3-3\end{array}$ & e & $\begin{array}{l}f \\
f\end{array}$ & & $\begin{array}{l}15 \\
75\end{array}$ & & & \\
\hline$A-611^{d}$ & 6800 & $c-3-4$ & e & $\begin{array}{l}\mathrm{f} \\
\mathrm{f}\end{array}$ & & 15 & & & \\
\hline$A-6158$ & $2 / 1 / 10$ & $c-4-1$ & e & f & & 75 & & & \\
\hline$A-611^{d}$ & 2500 & $c-4-2$ & e & f & & 15 & & & \\
\hline $\begin{array}{l}A-601^{d} \\
A-6011^{d}\end{array}$ & $\begin{array}{l}2960 \\
6790\end{array}$ & $\begin{array}{l}c-4-3 \\
i-4-4\end{array}$ & é & $\begin{array}{l}f \\
f\end{array}$ & $\dagger$ & $\begin{array}{l}75 \\
75\end{array}$ & & 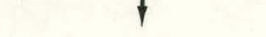 & \\
\hline
\end{tabular}

apercent conversion $=\frac{2-x}{2} \cdot 100$, where $x=$ atomic fraction of oxygen in kernel; e.g., UCy0 $0.5=75 \%$ converted.

$\mathrm{h}_{\mathrm{A}}=$ kernel; $\quad \mathrm{B}=$ butfer; $\quad \mathrm{C}=\mathrm{IPyC} ; \quad \mathrm{D}=\mathrm{SiC} ; \mathrm{E}=$ OPyC.

CMaximum fuel design temperature.

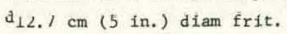

cPeak fast fluence w11l be $8 \times 10^{21} \mathrm{n} / \mathrm{cm}^{2}$ at end of irradiation period.

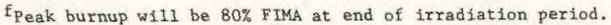

$812.7 \mathrm{~cm}$ (5 in.) diam cone.

${ }^{1}$ F. J. Homan et a1., Irradiation Performance of HTGR Fuel Rods in HFIR Experiments HRB-4 and -5, ORNL-5115, in publication.

2F. J. Homan et al., Irradiation Performance of HTGR Fuel Rods in HFIR Experiment HRB-6, ORNL-TM-5011 (December 1975).

${ }^{3}$ K. H. Valentine et al., Irradiation Performance of HTGR Fuel Rods in HFIR Experiments HRR-7 and -8, in preparation.

${ }^{4}$ HTGR Base Technology Program Arru. Progr. Rep. Jan. 1. 1974 through thme 30, 1975, Sect. 6.5.3, in preparatiun. 


\section{Table 3a. Results of Irradiation Tests on Weak-Acid Resin-Derived} Recycle Fuel

\begin{tabular}{|c|c|}
\hline Sample identification & Remarks ${ }^{a}$ \\
\hline \multicolumn{2}{|r|}{$\underline{\text { HRB }-5}$} \\
\hline $1 \mathrm{~A}, 1 \mathrm{~B}, 1 \mathrm{C}$ & \multirow[b]{2}{*}{$\begin{array}{l}\text { Matrix-particle interaction recognized as a potential } \\
\text { problem with high pitch-coke yields. No amoeba. Kernel } \\
\text { densification apparent. }\end{array}$} \\
\hline $\begin{array}{l}3 \mathrm{~A}, 3 \mathrm{~B} \\
3 \mathrm{C}, 3 \mathrm{D}\end{array}$ & \\
\hline \multicolumn{2}{|r|}{$\underline{\text { HRB }-4}$} \\
\hline $1 \mathrm{~A}, 1 \mathrm{~B}, 1 \mathrm{C}$ & $\begin{array}{l}\text { Extruded rods; carbonized in covered graphite tray; } \\
\text { fine cracks in SiC observed; no amoeba. }\end{array}$ \\
\hline $3 \mathrm{~A}, 3 \mathrm{~B}$ & \begin{tabular}{l|l} 
Slug-injected rods; carbonized in packed $\mathrm{Al}_{2} \mathrm{O}_{3}$. & $\begin{array}{l}\text { Attack of } \\
\text { observed. }\end{array}$ Phase segregation in about $25 \%$ of the kernels.
\end{tabular} \\
\hline 3C, 3D & Metallographic results indicate not all particles were $95 \%$ conve \\
\hline \multicolumn{2}{|r|}{ ( } \\
\hline $1 \mathrm{C} 4,105$ & $\begin{array}{l}\text { Loose particles in graphite tube; recovery of all particles not possible; majority failed. } \\
\text { Loose particles in graphite tube; tube broke during irradiation; particles lost. }\end{array}$ \\
\hline $1 \mathrm{C} 6$ & Loose particles in graphite tube; tube broke during irradiation; particles lost. \\
\hline \multicolumn{2}{|r|}{$\underline{\text { HRB }-7}$} \\
\hline 13 & Slug-injected rod; no failures (Metallography); buffer consumed; slight attack of SiC by rare earths. \\
\hline 14 & Slug-injected rod; $\sim 50 \%$ failed (Metallography); ILTI graphitized by rare earths on cold side. \\
\hline 15 & $\begin{array}{l}\text { Slug-injected rod; no failures (Metallography); buffer consumed; phase segregation in kernel; no evidence of rare earth } \\
\text { in coatings. }\end{array}$ \\
\hline 16 & $\begin{array}{l}\text { Slug-injected rod; no failures (Metallography); unilateral movement of kernel through buffer; no evidence of rare earth } \\
\text { in coatings. }\end{array}$ \\
\hline \multicolumn{2}{|r|}{$\underline{\text { HRB }-8}$} \\
\hline 13 & Slug-injected rod; no failures (Metallography); buffer consumed; slight attack of S1C by rare earths. \\
\hline 14 & Slug-injected rod; all failed (Metallography); ILTI graphitized by rare earths on cold side. \\
\hline 15 & $\begin{array}{l}\text { Slug-injected rod; no failures (Metallngraphy); buffer consumed; phase segregation in kernel; no evidence of rare earth } \\
\text { in coatings. }\end{array}$ \\
\hline 16 & $\begin{array}{l}\text { Slug-injected rod; no failures (Metallography); kernel densification apparent; buffer intact; no evidence of rare earth } \\
\text { in coatings. }\end{array}$ \\
\hline \multicolumn{2}{|r|}{$\underline{\text { HRB-9 }}$} \\
\hline $1,3,4,5$ & \multirow{2}{*}{$\begin{array}{l}\text { Slug-injected rod; stereo-exam only. No broken particles on surface. } \\
\text { Slug-injected rod; } \sim 20 \% \text { failed (Metallography); buffer in half of particles densified and breached; two-phase kernel; } \\
\text { Slight accumulation of rare earths on cold side; no graphitization of ILTI; slight attack of S1C. }\end{array}$} \\
\hline 6 & \\
\hline 8 & Slug-injected rod; no failures (Metallography); buffer consumed; phase segregation in kernel. \\
\hline 9 & $\begin{array}{l}\text { Slug-injected rod; no failures (Metallography); buffer intact; phase segregation in kernel; evidence of rare earth } \\
\text { accumulation on cold side; no graphitization of ILTI; no attack of SiC }\end{array}$ \\
\hline 10 & $\begin{array}{l}\text { Warm-molied rod; no faflures (Metallography); buffer intact; phase segregation in kernel; less evidence of rare earth } \\
\text { accumulation 1n rod } 9 \text {; wu graphitization of ILTI; no attack of SiC. }\end{array}$ \\
\hline 11 & $\begin{array}{l}\text { Slug-injected rod; } 275 \% \text { failed (Metallography); accumulation of rare earth on cold side; graphitization of ILTI; } \\
\text { no attack of SiC; two-phase kernel. }\end{array}$ \\
\hline 12 & $\begin{array}{l}\text { Slug-injected rod; no failures (Metallography); amoeba through buffer; rare earths retained in kernel; slight attack of } \\
\text { Sic on hot side }(2 \mu \mathrm{m}) \text {. }\end{array}$ \\
\hline 13 & $\begin{array}{l}\text { Slug-injected rod; no failures (Metallography); buffer intact but densif led around kernel; phase segregation in kernel; } \\
\text { accumulation of rare earths on cold side; no graphitization of ILTI; no attack of SiC. }\end{array}$ \\
\hline 14 & $\begin{array}{l}\text { Slug-injected rod; } \sim 50 \% \text { failed (Metallography); phase segregation in kernel; accumulation of rare earths on cold side; } \\
\text { graptilization of ILII; attack of SiC }(\sim 5 \mathrm{~m}) \text {. }\end{array}$ \\
\hline 15 & slug-infected rod; stereo-exam only; no failures. \\
\hline 16 & Slug-injected rod; no failures (Metallography); buffer consumed; phase segregation in kernel. \\
\hline 17 & Slug-injected rod; same remarks as for HRB-9, Rod 13 ( $50 \%$ converted). \\
\hline 18 & Slug-injected rod; stereo-exam only; no broken particles. \\
\hline 19 & Slug-1njected rod; stereo-exam only; no broken particles. \\
\hline & $\underline{\text { HRB-10 }}$ \\
\hline 1 & Slug-injected rod; not examined, due to reaction with thermocouple. \\
\hline 3 & Slug-injected rod; stereo-exam only; no broken particles. \\
\hline 4,5 & Slug-1njected rod; steteu-exam unily; ho brolecr particloo. \\
\hline 6 & $\begin{array}{l}\text { Slug-injected rod; } ~ 30 \% \text { failed (Metallography); accumulation of rare earths on cold side; graphitization of ILTI and } \\
\text { buffer; attack of SiC with complete penetration. }\end{array}$ \\
\hline 8 & Slug-injected rod; no failures (Metallography); buffer consumed; phase segregation in kernel. \\
\hline 9 & $\begin{array}{l}\text { Slug-injected rod; } \sim 8 \% \text { failed (Metallography); phase segregation in kernel; accumulation of rare earths on cold side. } \\
\text { slight graphitization of ILTI; no attack of SiC. }\end{array}$ \\
\hline 10 & $\begin{array}{l}\text { Warm-molded rod; no failures (Metallography); phase segregation in kerne1; accumulation of rare earths on cold side; } \\
\text { no graphitization of ILTI; slight attack of SiC. }\end{array}$ \\
\hline 11 & $\begin{array}{l}\text { Slug-injected rod; } \sim 18 \% \text { failed (Metallography); two-phase kernel; accumulation of rare earths on cold side; } \\
\text { graphitization of ILTI; attack of SiC }(\sim 5 \mu \mathrm{m}) \text {. }\end{array}$ \\
\hline 12 & $\begin{array}{l}\text { Slug-1njected rod; i } 5 \% \text { falled (Metallography); amoeba through buffer; rare earths retained in kernel; attack of SiC } \\
\text { isigil to cumplele pruetration). }\end{array}$ \\
\hline 13 & $\begin{array}{l}\text { Slug-injected rod; no failures (Metallography); same remarks as for HRB-9, Rod } 13 \text { ( } 50 \% \text { converted), except more evidence } \\
\text { of rare-earth accumulation. }\end{array}$ \\
\hline 14 & $\begin{array}{l}\text { Slug-infected rod; } \sim 30 \% \text { fallures (Metallography); two-pnase iceruel, accumulation of rare earths on cold side; } \\
\text { graphitization of ILTI; attack of SiC (complete penetration). }\end{array}$ \\
\hline
\end{tabular}


Table 3a. (Continued)

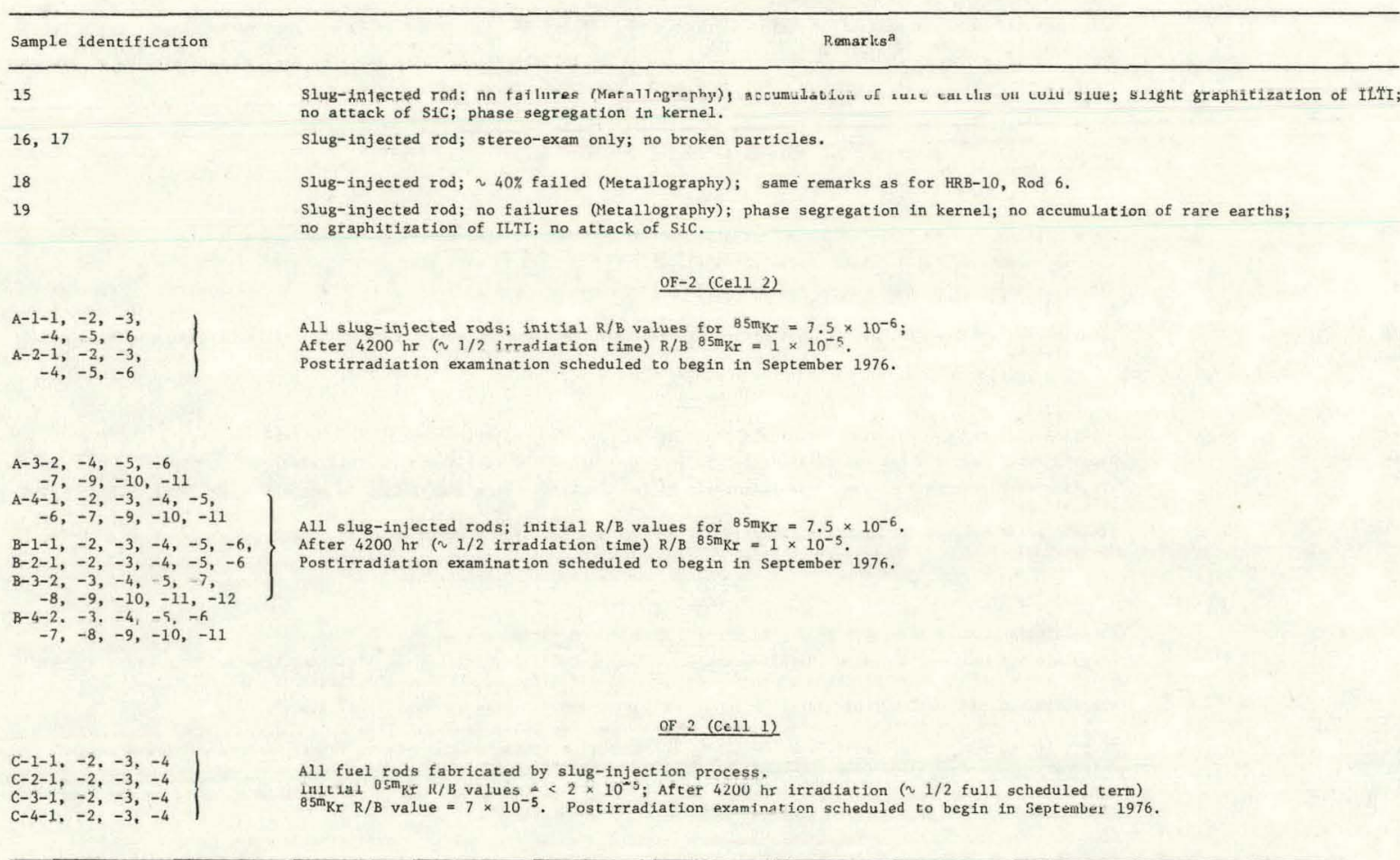

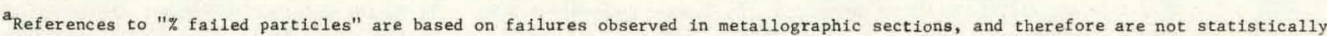
significant since normally $<20$ fissile particles are exposed in a random plane-of-polish. These results are, however, indicators of relative performance. 

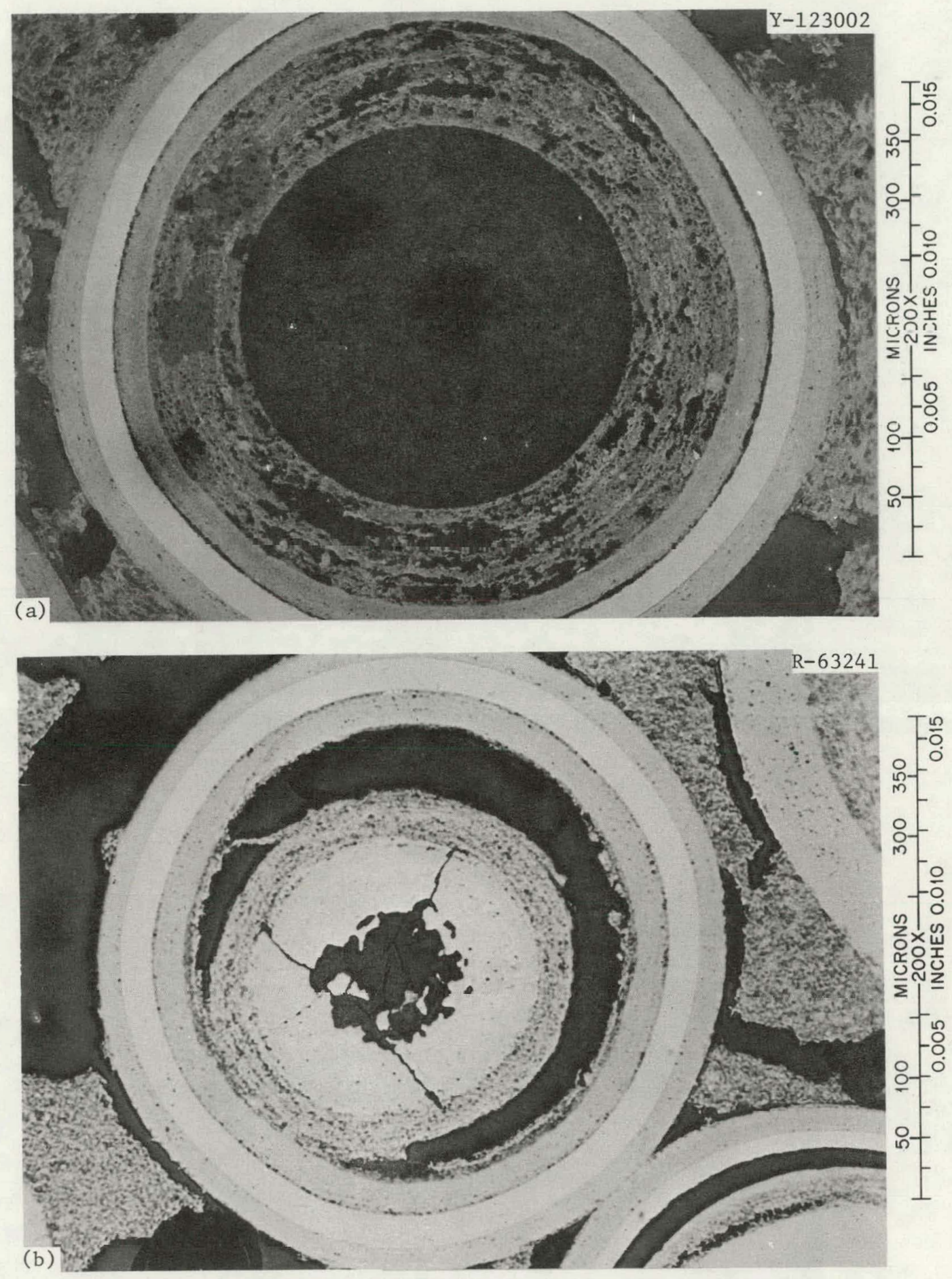

Fig. 8. Weak-Acid Resin Particles Used in Slug-Injected Fuel Rods for Irradiation Testing. (a) Unirradiated. (b) Irradiated in HRB-5, rod 3B. Typical of particles in the cross section of the fuel rod. Time-averaged temperatures ranged from 1150 to $1400^{\circ} \mathrm{C}$ with peak temperature of $1525^{\circ} \mathrm{C}$. 

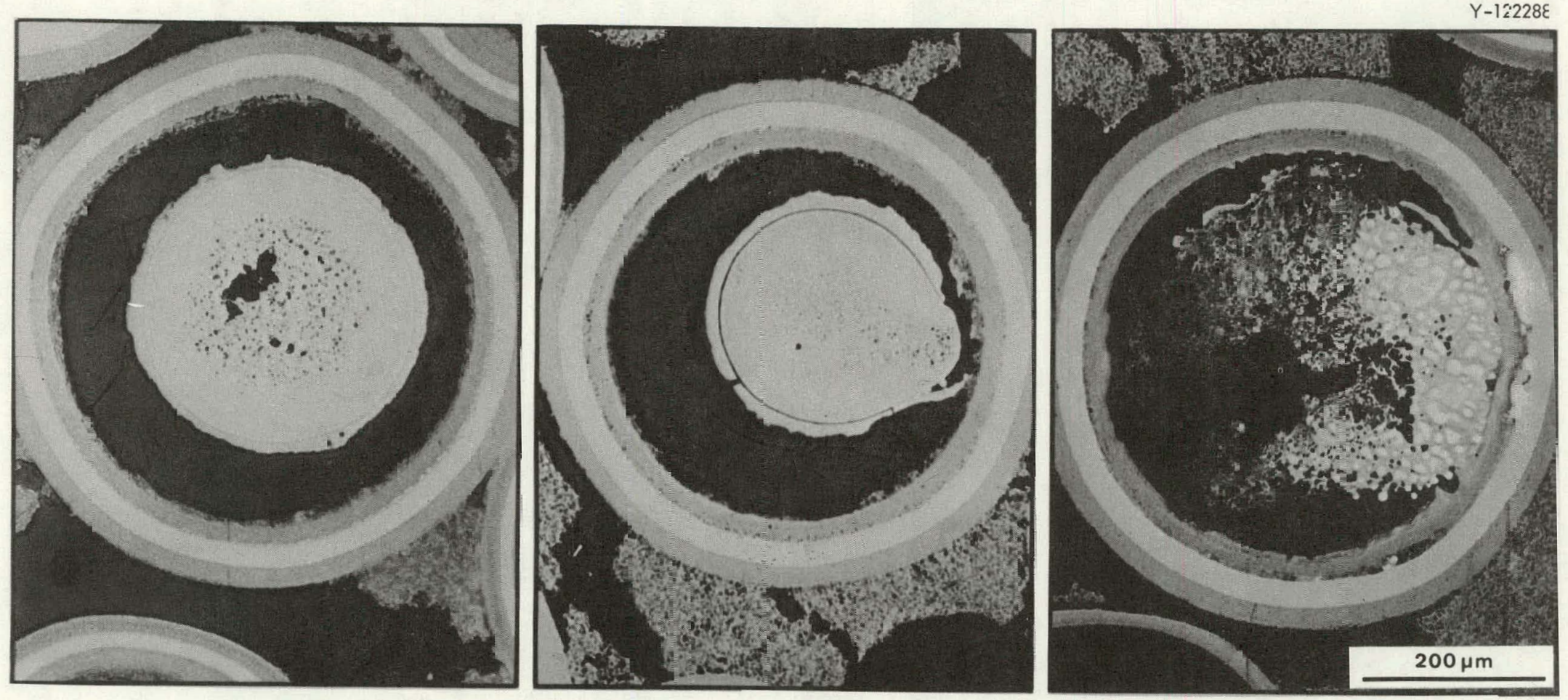

Fig. 9. Appearance of Weak-Acid Resin Fissile Coated Particles From HRB-4, SIug-Injected Specimen 3B. 


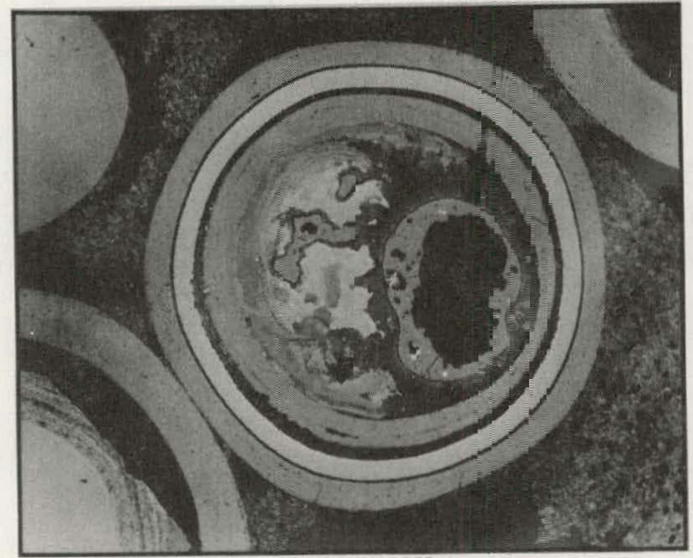

$0 \%$ CONVERTED
$\mathrm{R}-70001$

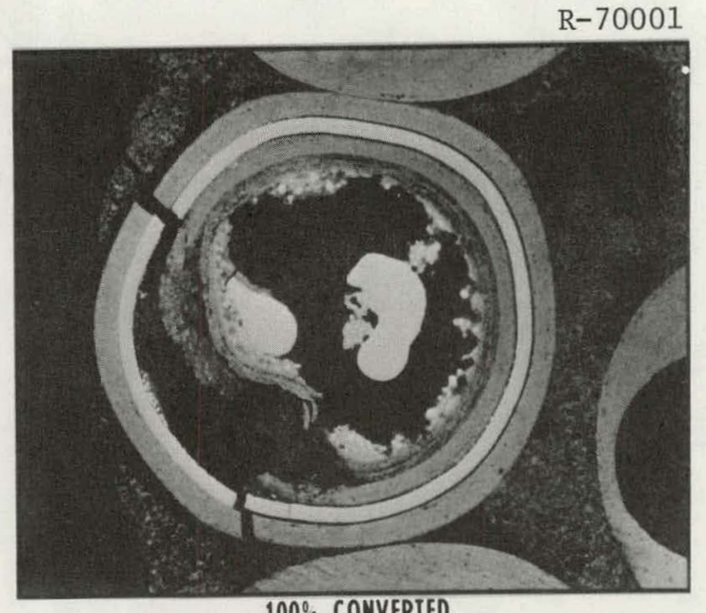

$100 \%$ CONVERTED

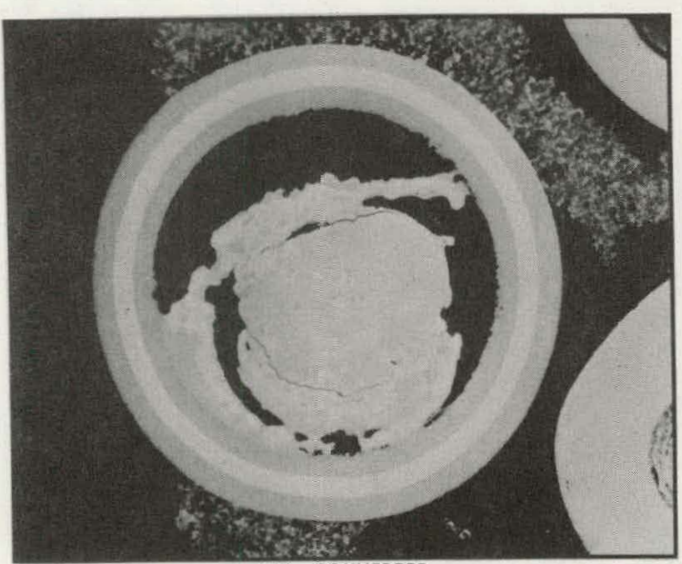

$15 \%$ CONVERIED

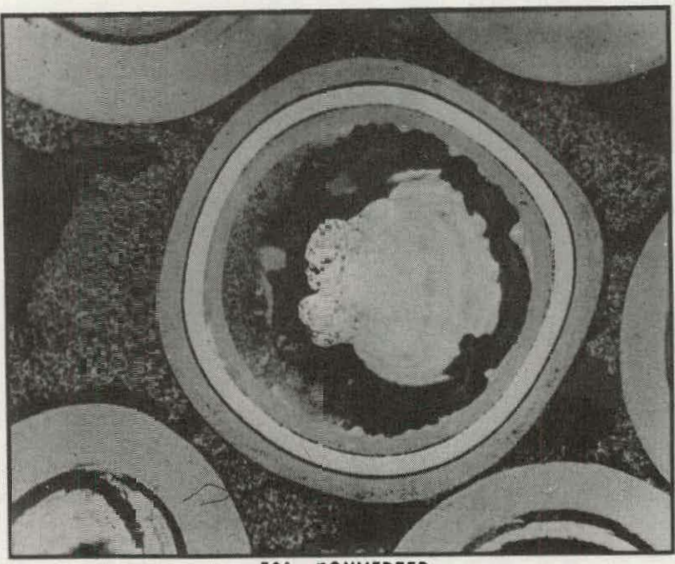

$50^{\circ}$, CONVERTED

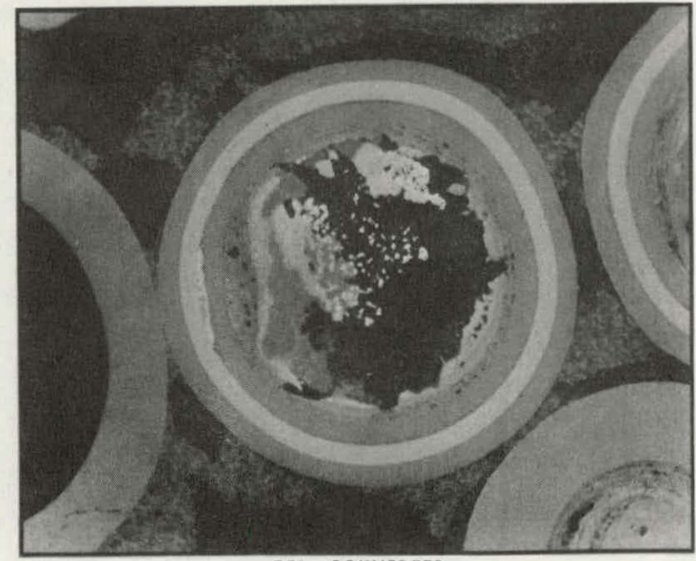

$75^{\circ}$ CONVERTED

Fig. 10. Relative Performance ố hieak-Acid Derived Kernels Converted Within the Range of $\mathrm{UO}_{2}$ ( $0 \%$ Converted) to $\mathrm{UC}_{2}\left(100 \%\right.$ Converted). Irradiated at a maximum fuel temperature of $1500^{\circ} \mathrm{C}$ to a turnup of $80 \%$ FIMA. Fast fluence ranzec from 4.3 to $5.0 \times 10^{21} \mathrm{n} / \mathrm{cm}^{2}$ (E $\left.>0.18 \mathrm{MeV}\right)$. The two Farticles at the top of the figure ar $\equiv$ from the peripheral regions of fuel rods and operated at less than peak rod temperatures but demonstrate performance limitations in steep thermal gradient $\left(>800^{\circ} \mathrm{C} / \mathrm{cm}\right)$ conditions. The hot side of the particles is on the right. 

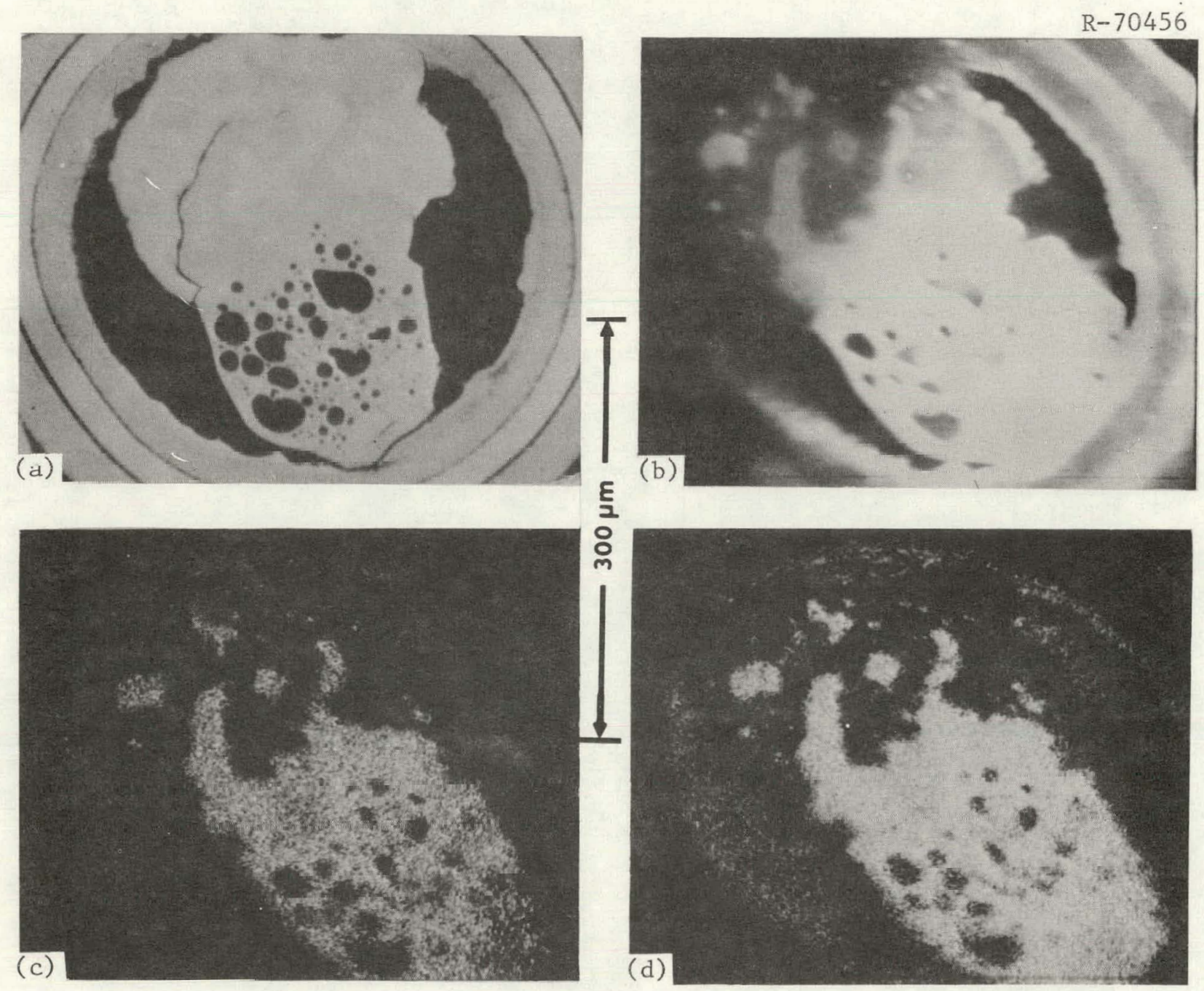

Fig. 11. Distribution of Uranium and Rare-Earth Fission Products (Typified by Nd X-Ray Disp $1 \mathrm{ay}$ ) in a $15 \%$ Converted Weak-Acid Resin-Derived Particle. There is no indication that the Nd penetrated the inner pyrocarbon layer. (a) Optical micrograph. (b) Backscattered electron image. (c) $\mathrm{U} \mathrm{M}_{\beta} \cdot$ (d) $\mathrm{Nd} \mathrm{L}_{\alpha}$. 

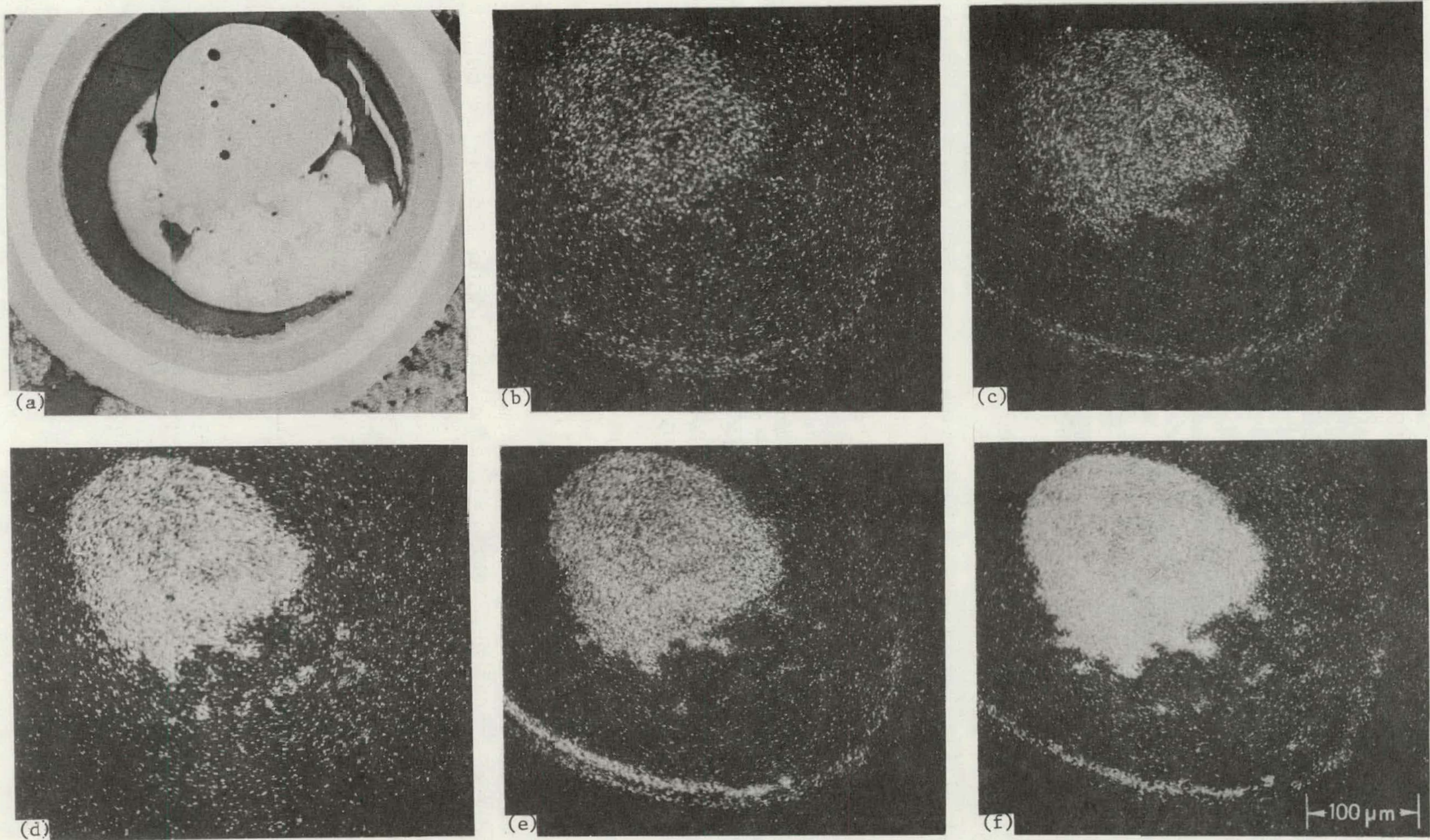

Fig. 12. Distribution of Uranium and Rare-Earth Fission Products in a Triso-Coated 15\% Converted Weak-Acid Resin Particle Irradiated in HRB-8. (a) Optical micrograph. (c) $\mathrm{U} \mathrm{M}_{\beta} \cdot$ (e) $\mathrm{Ce} \mathrm{L}_{\alpha}$. (f) $\mathrm{Nd} \mathrm{L}_{\alpha}$. 
4. There is considerable fission product attack of the SiC coatings by rare-earth fission products $\mathrm{La}, \mathrm{Ce}, \mathrm{Pr}$, and $\mathrm{Nd}$ in $\mathrm{WAR} \mathrm{UC}_{2}$ fuels. This same type of attack has been observed in dense $\mathrm{UC}_{2}$ fuels irradiated in HRB capsules under accelerated conditions, and also in the Peach Bottom Reactor under real-time conditions. The presence of $\mathrm{UO}_{2}$ reduces the amount of fission product attack of the SiC layer, apparently due to the formation of stable rare-earth oxides, which are retained in the kernel. Optical metallography and shielded microprobe displays of nomina11y $95 \% \mathrm{UC}_{2}$ fuel irradiated in the HRB-4 capsule are shown in Fig. 13. A similar display of reference fresh fissile fuel ( $\mathrm{UC}_{2}$ ) irradiated under real-time conditions in the Peach Bottom Reactor is shown in Fig. 14. (Note that the $\mathrm{UC}_{2}$ shown in Fig. 14 has been irradiated to less than $20 \%$ full burnup and to less than $20 \%$ of the fast neutron expnsure experted for the LHTCR. ${ }^{14}$ ) The similarity of risslun product deposition in WAR $\mathrm{UC}_{2}$ and dense $\mathrm{UC}_{2}$ fuel irradiated in $\mathrm{HRB}-7$ is shown in Fig. 15. The polarized-light portion of this figure reveals regions having a high degree of optical activity, which indicates graphitization as a result of fission product interaction.

5. There is a considerable temperature gradient effect on the fission product attack. Under high-temperature gradients, the fission products are concentrated on the cold side of the particle, and failure of the SiC layer is likely, as shown on the left side of Fig. 16. Under less severe temperature gradients, the fission products are less concentrated and do less damage. The particle shown on the right side of Fig. 16 was operated at the center of the same fuel rod. The temperature gradient is zero at the center of the rod, and even though the operating temperature was higher at this position, less damage was done to the coatings by the fission products. The bottom portion of Fig. 16 (in polarized light) shows the light regions where fission products are present. 'The temperature gradient of $1000^{\circ} \mathrm{C} / \mathrm{cm}$ is about $30 \%$ higher than the maximum temperature gradient for an LHTGR (Table 1).

Fission product attack of the coatings appears to be the limiting performance feature of $\mathrm{UC}_{2}$ fuels. Thermal migration appears to be limiting for $\mathrm{UO}_{2}$. Intermediate stoichiometries appear to perform well compared with these extremes. In the HRB-7 through -10 series of experiments, WAR fissile fuels with conversion levels of $0,15,50$, 75 , and $100 \%$ were tested. These WAR kernels were coated with reference Triso coatings and were fabricated into fuel rods using the olug injection technique. As indicated earlier, the WAR $\mathrm{IT}_{2}$ kernel has migraced up the temperature gradient, and the WAR $\mathrm{UC}_{2}$ particle has failed due to fission product attack of the SiC layer. Figure 17 shows an optical photomicrograph of a WAR $\mathrm{UC}_{2}$ particle irradiated in $\mathrm{HRB}-8$. Also shown are electron microprobe displays for the rare-earth fission products $\mathrm{La}, \mathrm{Pr}, \mathrm{Ce}$, and $\mathrm{Nd}$. The graphitization of the inner pyrolytic carbon coating and failure of the SiC in the regions occupied by the fission products indicates failure due to chemical interaction. 

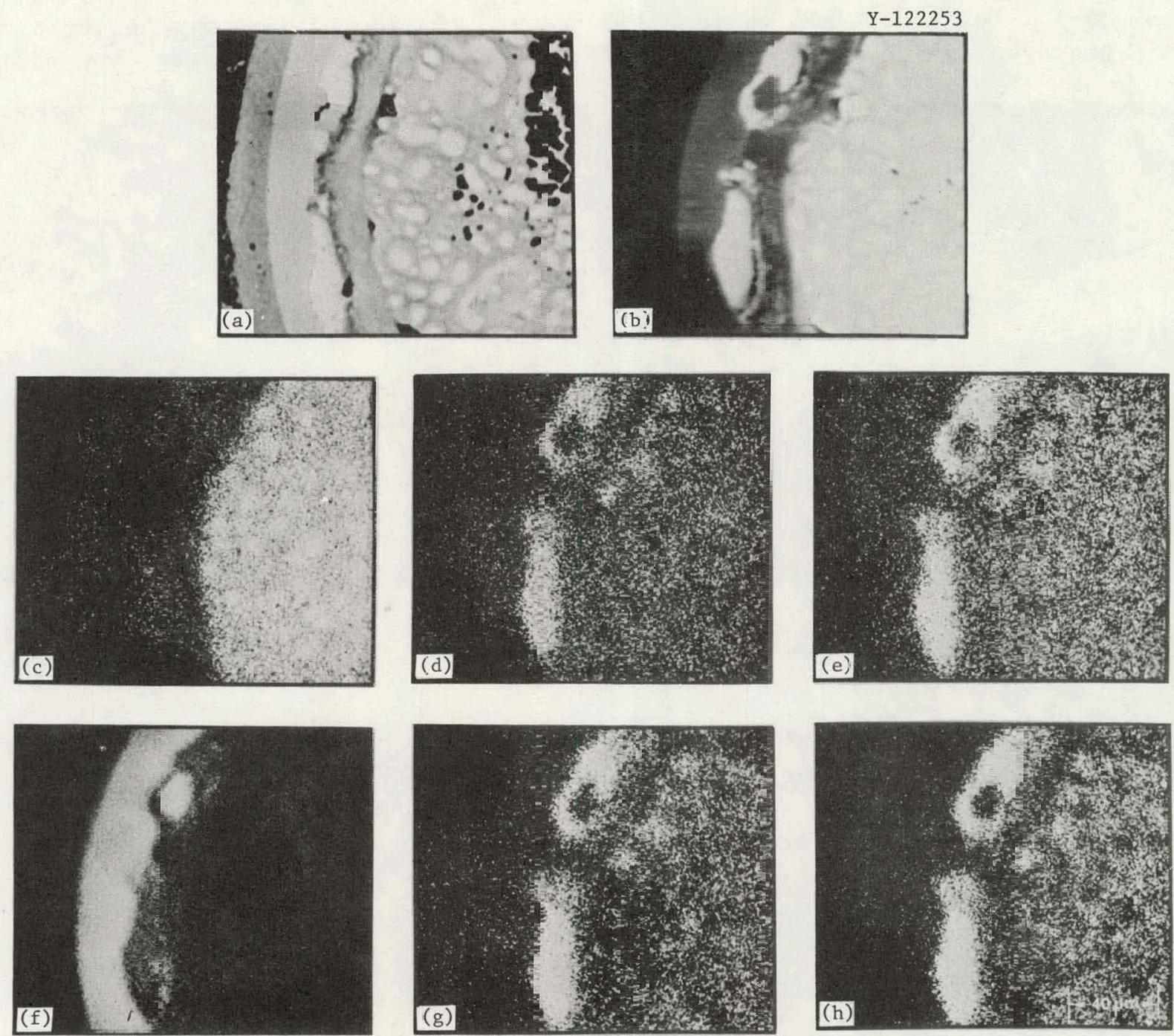

Fig. 13. Weak-Acid Resin Particle From Fuel Rod 3B, Irradiction Capsule HzB-4. (a) Optical photograph. (b) Backscattered electron. (c) U $\mathrm{M}_{\alpha}$. (d) $\mathrm{La} \mathrm{L}_{\alpha} \cdot \quad$ (e) $\operatorname{Pr} \mathrm{L}_{\alpha}$. (f) $\mathrm{Si} \mathrm{L}_{\alpha}$. (g) $\mathrm{Ce}_{\alpha}$. (h) Nd $\mathrm{L}_{\alpha}$. 

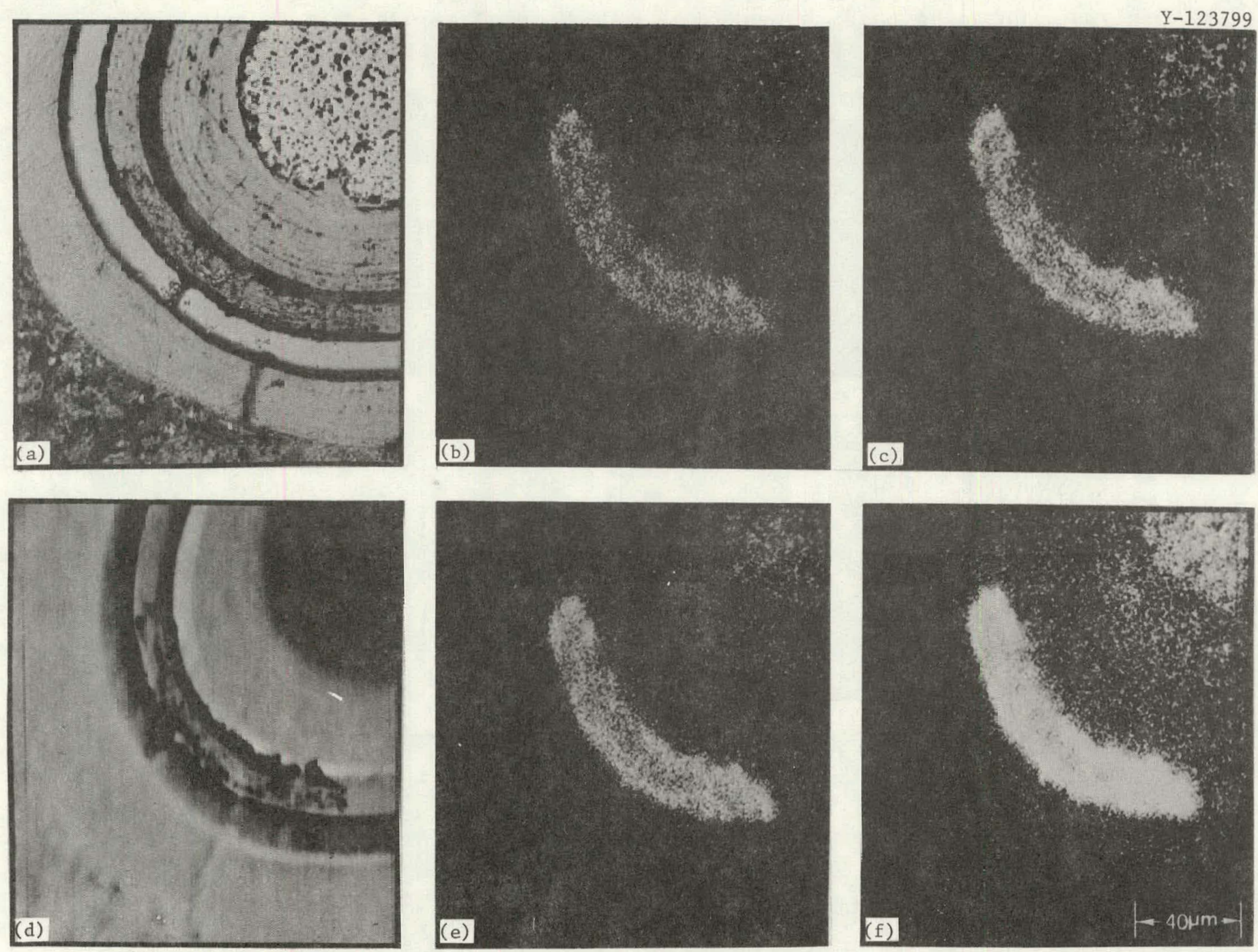

Fig. 14. Rare-Eerth Fission Products Found on Cold Side of Trisa-Coated Dense $\mathrm{UC}_{2}$ Kernel from RTE-4. (a) Optical micrograph. (b) La $\mathrm{L}_{\alpha}$. (c) $\operatorname{Pr} \mathrm{L}_{\mathrm{C} \cdot} \cdot$ (d) Backscattered electrons. (e) $\mathrm{Ce} \mathrm{L}_{\alpha}$. (f) $\mathrm{Nd} \mathrm{L}_{\alpha}$. 

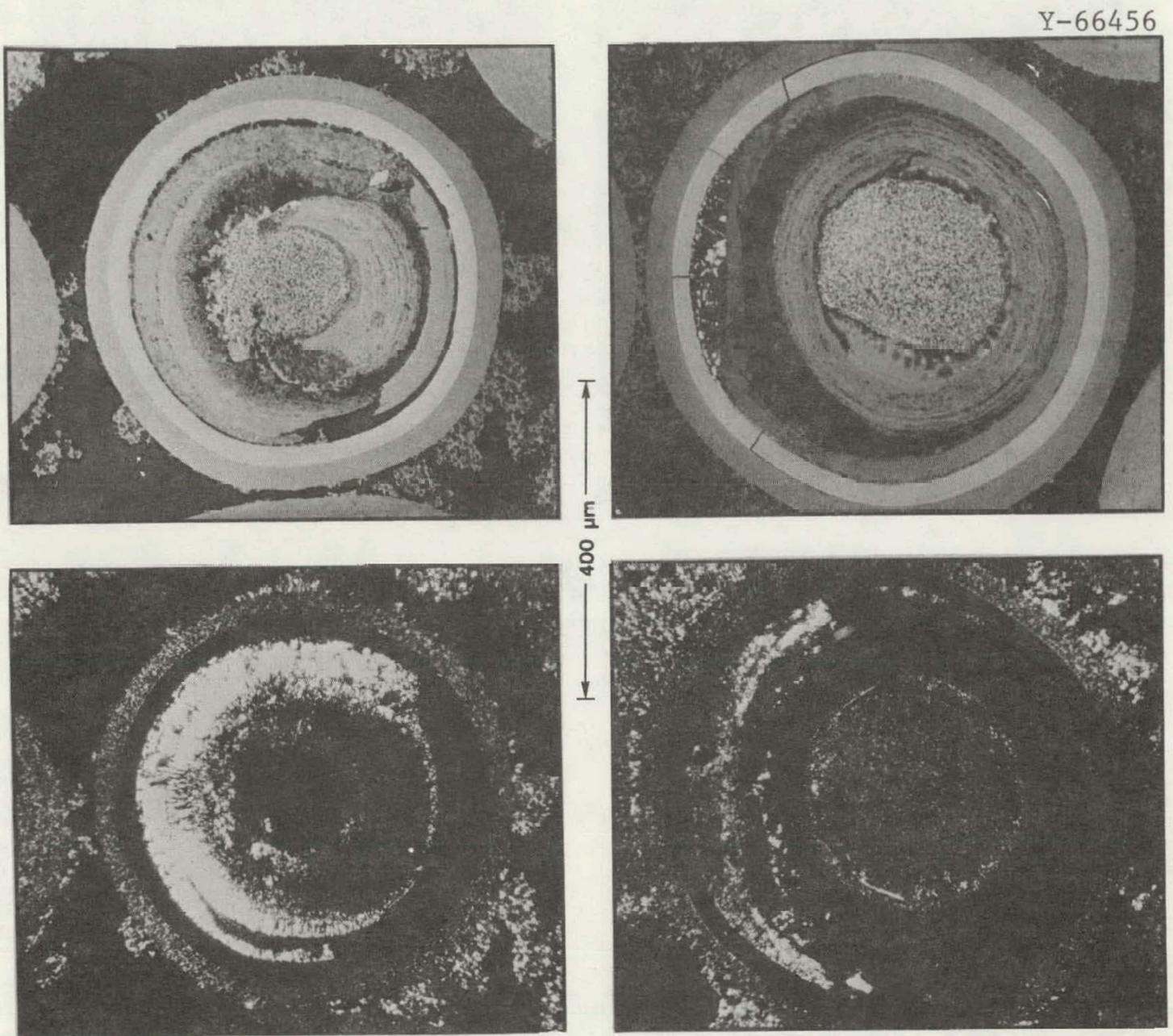

Fig. 15. Relative Performance of Dense $\mathrm{UC}_{2}$ and $W A R \mathrm{UC}_{2}$ in Irradiation Capsule HRB-7. Top: Bright field. Bottom: Polarized light. Left: $\mathrm{UC}_{2}$ (VSM). Right: WAR $-100 \%$ reduced. 
TEMPERATURE: $1100^{\circ} \mathrm{C}$
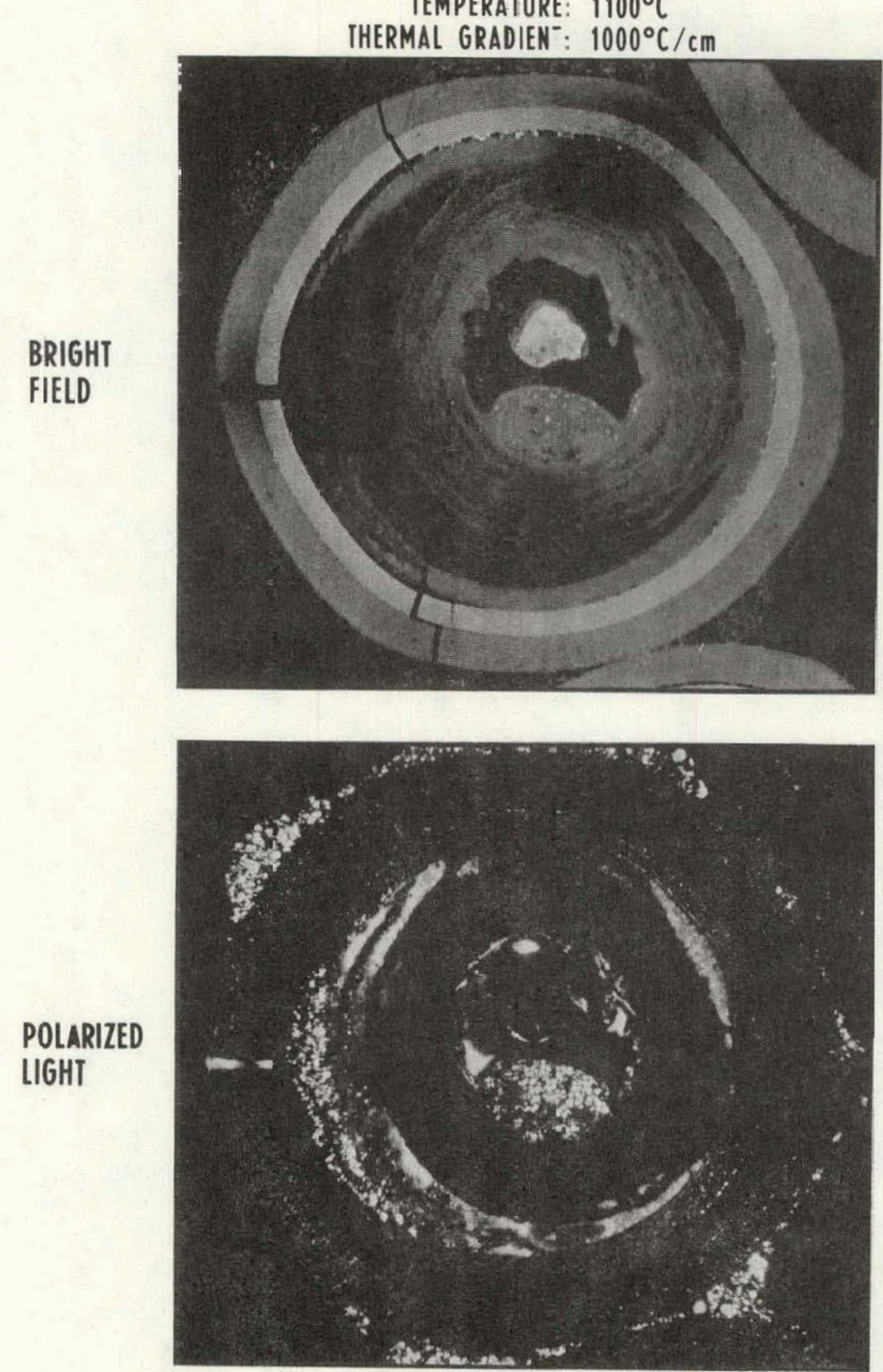

TEMPERATUR:. $1250^{\circ} \mathrm{C}$ THERMAL GRADIENT: $0^{\circ} \mathrm{C} / \mathrm{cm}$

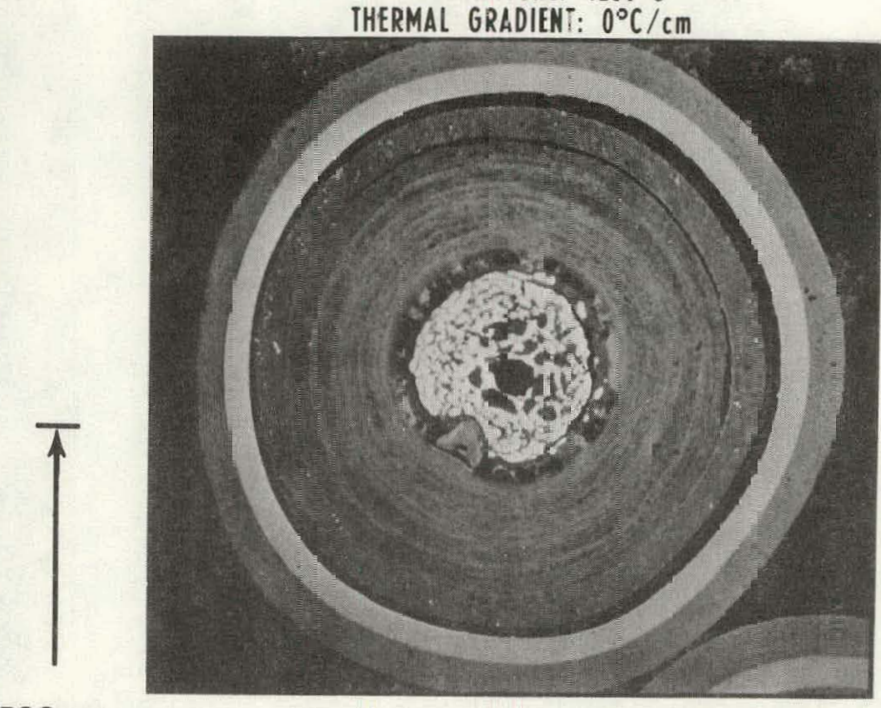

$500 \mu \mathrm{m}$

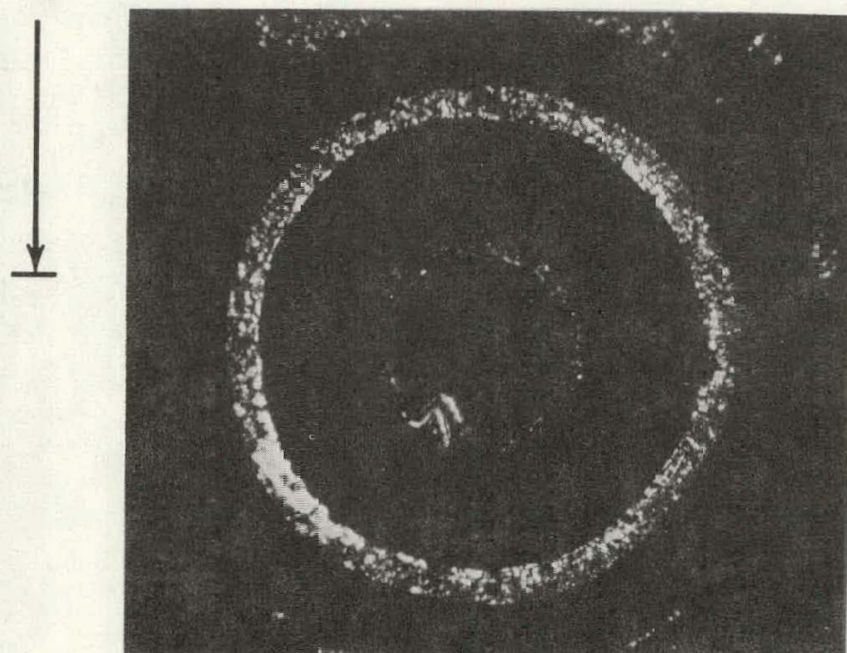

Fig. 16. Effect of Thermal Gradient on Distribution of Rare-Earth Fission Products in a Triso-Coated $100 \%$ Converted WAR UC 2 Kernel. 

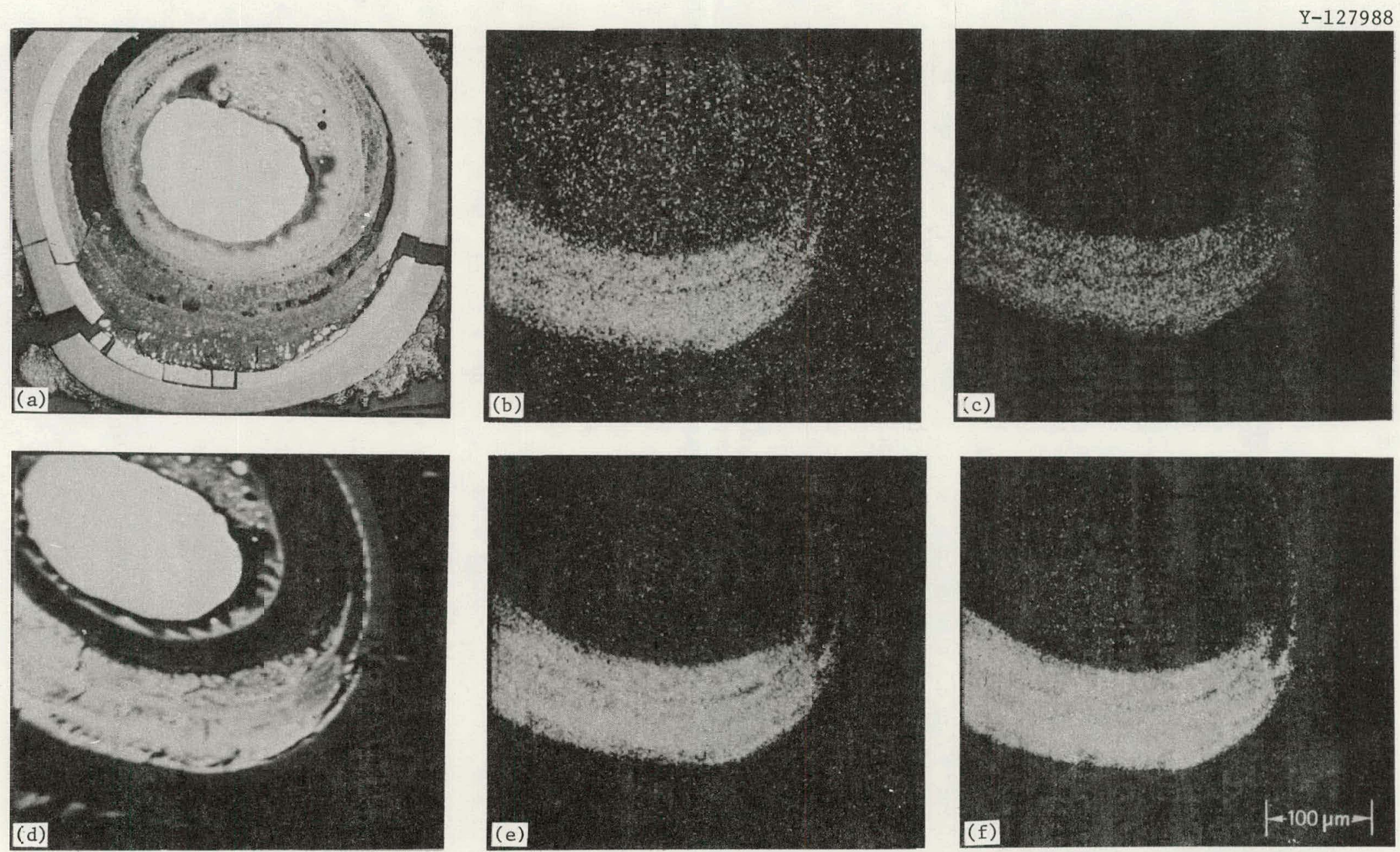

Fig. 17. Distribution of Rare-Earth Fission Products in a Triso-Coated $100 \%$ Reduced Weak-Acid Resin Particle Irradiated in $\mathrm{HRB}-8$. scattered electron image. (e) Ce $\mathrm{L}_{\alpha}$. (f) $\mathrm{Nd} \mathrm{L}_{x}$. scattered electron image. (e) $\mathrm{Ce} \mathrm{L}_{\alpha}$. (f) $\mathrm{Nd} \mathrm{L}_{x}$.

(a) Optical micrograph.

(b) $\mathrm{La} \tau_{\alpha}$.

(c) $\operatorname{Pr} L_{\alpha}$.

(d) Back- 
The thermodynamic arguments associated with kernel stoichiometry are discussed in ref. 1. Calculations have been made of the amount of $\mathrm{UO}_{2}$ necessary to keep all the rare-earth fission products in the form of oxides in WAR fuels irradiated to full burnup [about $75 \%$ fissions per initial heavy-metal atom (FIMA)]. The irradiation results from HRB-7 through -10 support the conclusions reached in these calculations. $\mathrm{UO}_{2}$ fuel (Fig. 18) retains all the rare-earth fission products in the kernel in the form of stable oxides. The 15\% converted WAR fuel shown in Figs. 11 and 12 have also retained most of the rare-earth fission products ( $93 \%$ ) within the kernel. As the amount of $\mathrm{UC}_{2}$ in the kernel is increased, the capacity to hold the rare-earth fission products within the kernel is reduced, as shown in Figs. 19 and 20. The slight accumulation of neodymium at the inner surface of the SiC layer in $50 \%$ converted fuel is shown in Fig. 19. Most of the neodymium, however, has been retained in the kernel. The neodymium display in this figure is also representative of $\mathrm{La}, \mathrm{Pr}$, and $\mathrm{Ce}$. Figure 20 shows higher magnification views of the cold side of the rnatings for the 15, 50, and $75 \%$ converted fuels. Slight accumulations of fission products, believed to be rare earths, can be seen at the SiC-inner LTI interface for particles with 50 and $75 \%$ converted kernels. The amount of fission product accumulation increased with an increase in the percentage of $\mathrm{UC}_{2}$ present.

The question of optimum kernel stoichiometry for WAR fuels has not yet been answered. Experiments that have been designed to establish the optimum $\mathrm{UO}_{2}$ content are now in-reactor. Based on the results from HRB -7 through -10 and the thermodynamic calculations, ${ }^{1}$ it is anticipated that the optimum value will be about $35 \%$ conversion, with a range of $\pm 20 \%$ permitted. The arguments against high $\mathrm{UC}_{2}$ content are clear. Some problems have also been encountered with high $\mathrm{UO}_{2}$ content, in addition to the amoeba already noted. While no fission product attack of the SiC layer was noted with the WAR $\mathrm{UO}_{2}$ fuel, there was some evidence of oxidation of the inner surface of the SiC layer. The amount of attack was moderate for fuel temperatures of about $1250^{\circ} \mathrm{C}$, but for temperatures of about $1500^{\circ} \mathrm{C}$ there was complete penetration of the SiC. Examples of the oxidation of the $\mathrm{SiC}$ in $\mathrm{WAR} \mathrm{UO}_{2}$ fuel are shown in Fig. 21. Fuel of this same composition was irradiated in capsule HRB-9 at a design center line temperature of $1250^{\circ} \mathrm{C}$, and very slight oxidation was noted.

From the processing standpoint, it is desirable to maximize the $\mathrm{UO}_{2}$ cunlent in the two phase WAk kenmels. Cunsiderable effort has therefore been expended to understand the behavior of the $15 \%$ converted fuel from HRB-7 through -10 , which is currently thought to be the lower limit for the conversion specification. ${ }^{1}$ Phase segregation shown in Figs. 11 and 12 is apparent both from optical examination and from microprobe work. 'l'he uranium-bearing phase is retained within the outer boundary of the buffer layer. It is apparent from the work done to date on $15 \%$ converted fuel that the buffer layer is largely incorporated into the kernel. According to the current definition of failure, developed for dense fissile kernels, this fuel would be unacceptable. 

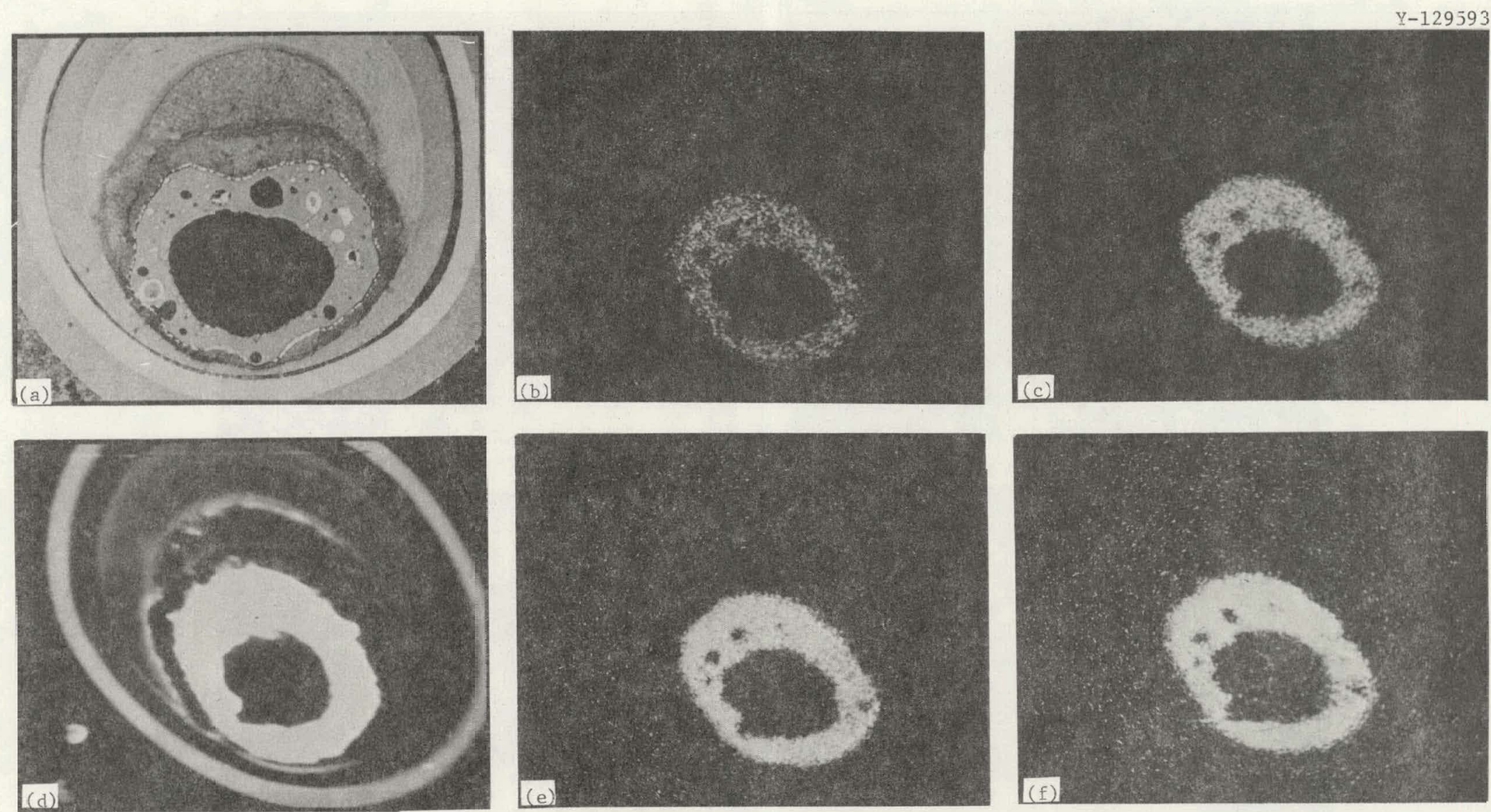

Fig. 18. Retention of Rare-Earth Fission Products in the Kernel of a Sol-Gel $\mathrm{UO}_{2}$ Triso-Coated Particle Irradiated in HRB-8. 180x. (a) Optical micrograph. (b) La $\mathrm{L}_{\alpha}$. (c) $\operatorname{Pr} \mathrm{L}_{\alpha}$. (d) Sample current image. (e) $\mathrm{Ce}_{\alpha}$. (f) $\mathrm{Nd} \mathrm{L}_{\alpha}$. 

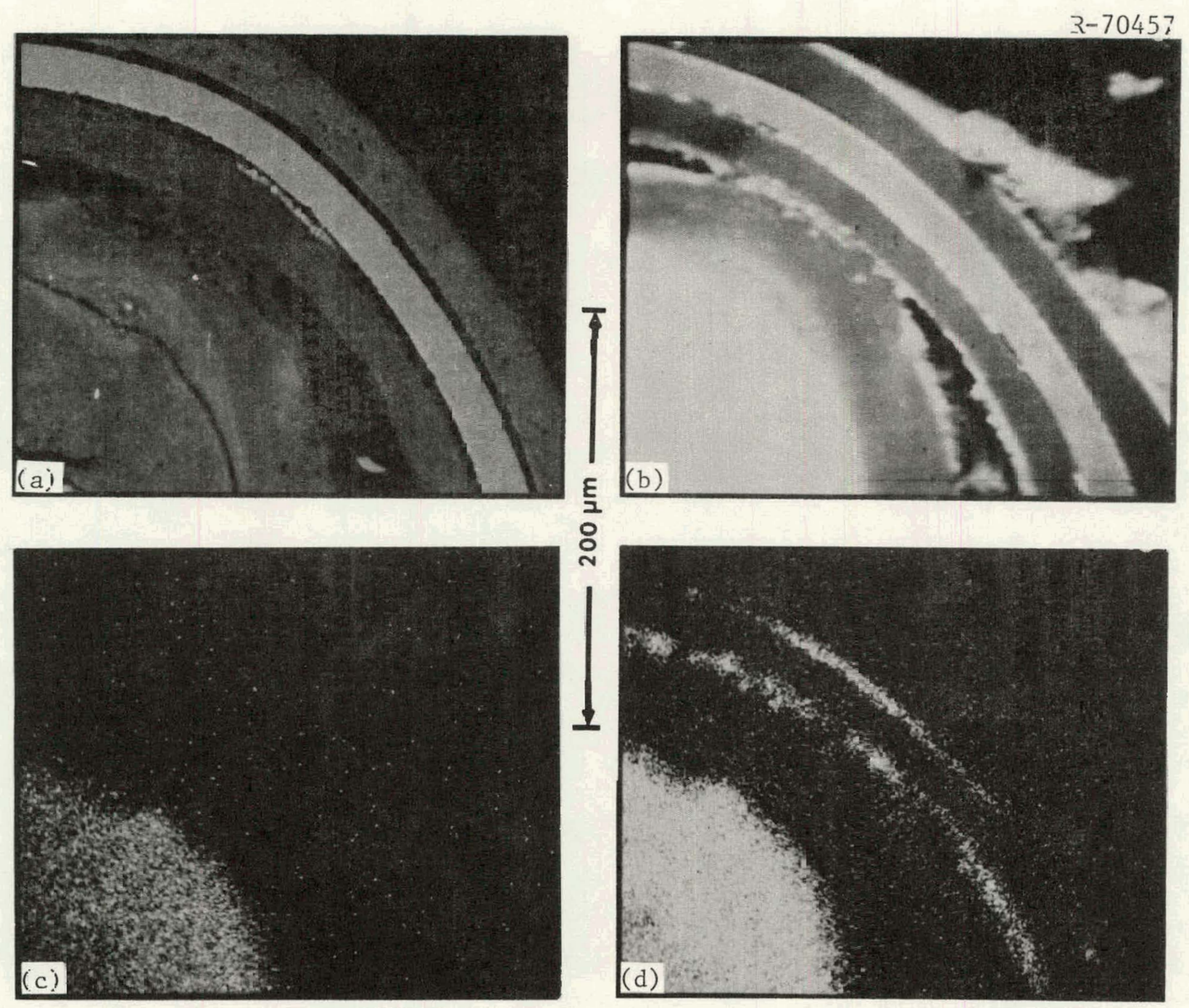

Fig. 19. Distribution of Uranium and Rare-Earta Fission Products

(Typified by Nd X-Ray Display) in a 50\% Converted Weak-f.cid-Derived Par-icle. Note that $\mathrm{Nd}$ is present at the inner surface of the JiC coating. (a) Optical micrograph. (b) Backscattered electron image. (c) $J \mathrm{M}_{E} \cdot$ (d) $\mathrm{Nd} \mathrm{L}_{\alpha}$. 

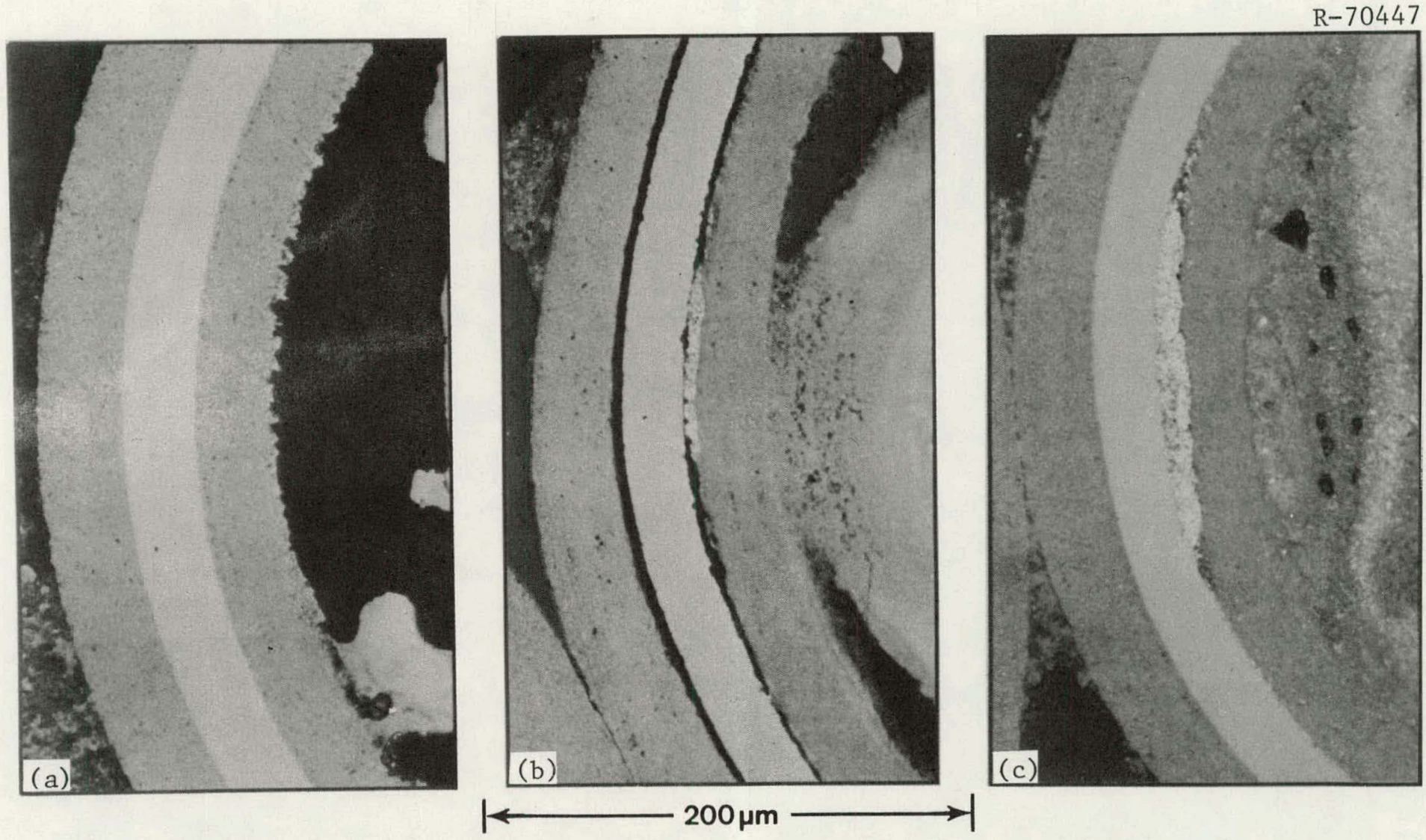

Fig. 20. Accumulation of Rare-Earth Fission Products at the SiC-Inner PyC Interface on the Cold Side of Triso-Coated Weak-Acid-Derived Kernels. Note the absence of fission products in the coatings of the $15 \%$ converted kernel. (a) $15 \%$ converted. (b) $50 \%$ converted. (c) $75 \%$ converted. 

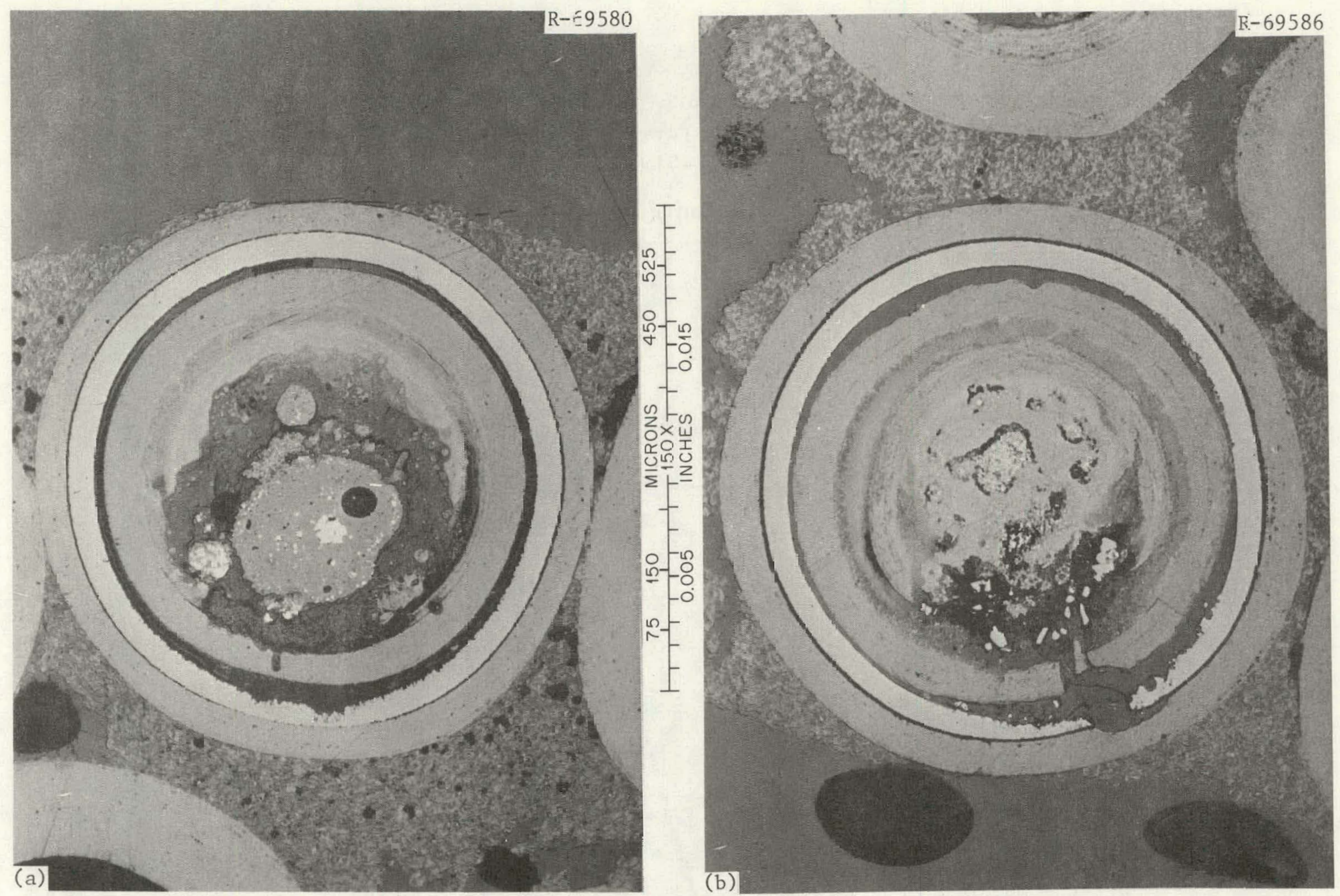

Fiz. 21. Oxidation of the SiC Layer in WAR UD 2 Fuel Irradiated in HRB-10.

(a) Irradiated on the surface of the fuel rod at ajout $1200^{\circ} \mathrm{C}$. (b) Irradiated at the center of the fuel rod at about $1500^{\circ} \mathrm{C}$. 
However, it is currently believed that this definition of failure is not appropriate for WAR fuels. For fissile particles containing dense oxide kernels that are migrating up the temperature gradient, migration through the buffer means that the integrity of the pressure vessel has been compromised. Thus, failure is defined as migration through the buffer layer. However, for fissile particles containing WAR $\mathrm{UO}_{2}-\mathrm{UC}_{2}$ kernels with more than the $15 \%$ carbide phase, temperature gradient dependent migration of the kernel has not been observed. The incorporation of the buffer layer into the kernel does not appear to influence the integrity of the outer coating layers. Irradiation testing is under way to prove statistically that the $15 \%$ converted WAR fuel meets the performance criteria for fissile particles. Quantitative electron microprobe results have shown that the $15 \%$ converted kernels retain all but about $7.5 \%$ of the rare-earth fission products that form during irradiation. Quantitative work is under way for kernels of 50 and $75 \%$ conversion, but it is certain that these fuels will release more fission products than the $15 \%$ converted fuel, based on the optical comparisons that have already been made.

\section{PERFORMANCE OF COATED PARTICLES AND FUEL RODS}

Of the five failure mechanisms for coated particle fuels 1isted in the Introduction, the first two have been described thoroughly in the previous sections of this report. The remaining three mechanisms will be described in this section.

Pressure vessel failure is only slightly dependent on the kernel type. As noted earlier, the WAR-derived kernels are considerably more porous than dense oxides or carbides and tend to shrink appreciably during irradiation. Both of these effects contribute to higher fission gas release at low burnup. High porosity fractions provide an easy route for fission gas release, and the kernel shrinkage and high porosity content contribute to higher kernel operating temperatures than would be experienced by dense kernels operating at the same power level. High operating temperatures drive more fission gas out of the kernel matrix. Pressure vessel failure is not considered to be a serious problem for recycle fissile fuel, since thicker SiC coatings that will withstand the higher fission gas release can be specified. An example of pressure vessel failure is shown in Fig. 22.

Matrix-particle interaction occurs when a strong bond forms between the matrix and the outer coating layer. Both matrix and coatings shrink during irradiation, causing the outer coating to fracture or separate. An example is shown in Fig. 23. The most exaggerated matrix-particle interaction seen was in the HRB-4 and -5 experiment ${ }^{12}$ where the fuel rods were carbonized in a graphite tube to simulate in-block carbonization. No interaction was observed in fuel rods carbonized in beds of packed alumina; presumably because the volatile hydrocarbons are more easily removed. 


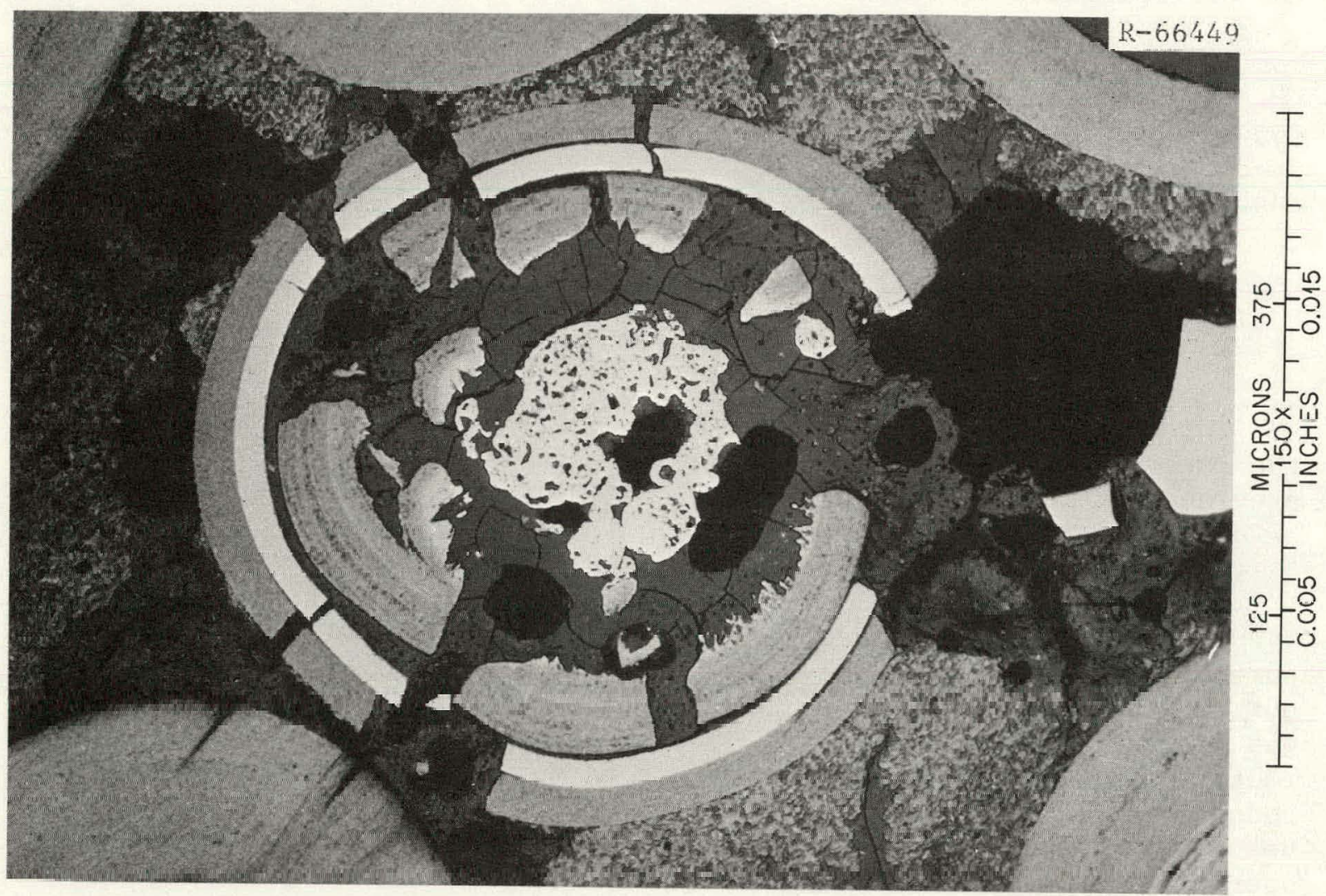

Fig. 22. Pressure Vessel Failure in $\mathrm{HRB}-8$, Specimen $5\left({ }^{23} \mathrm{UO}_{2}\right)$. 

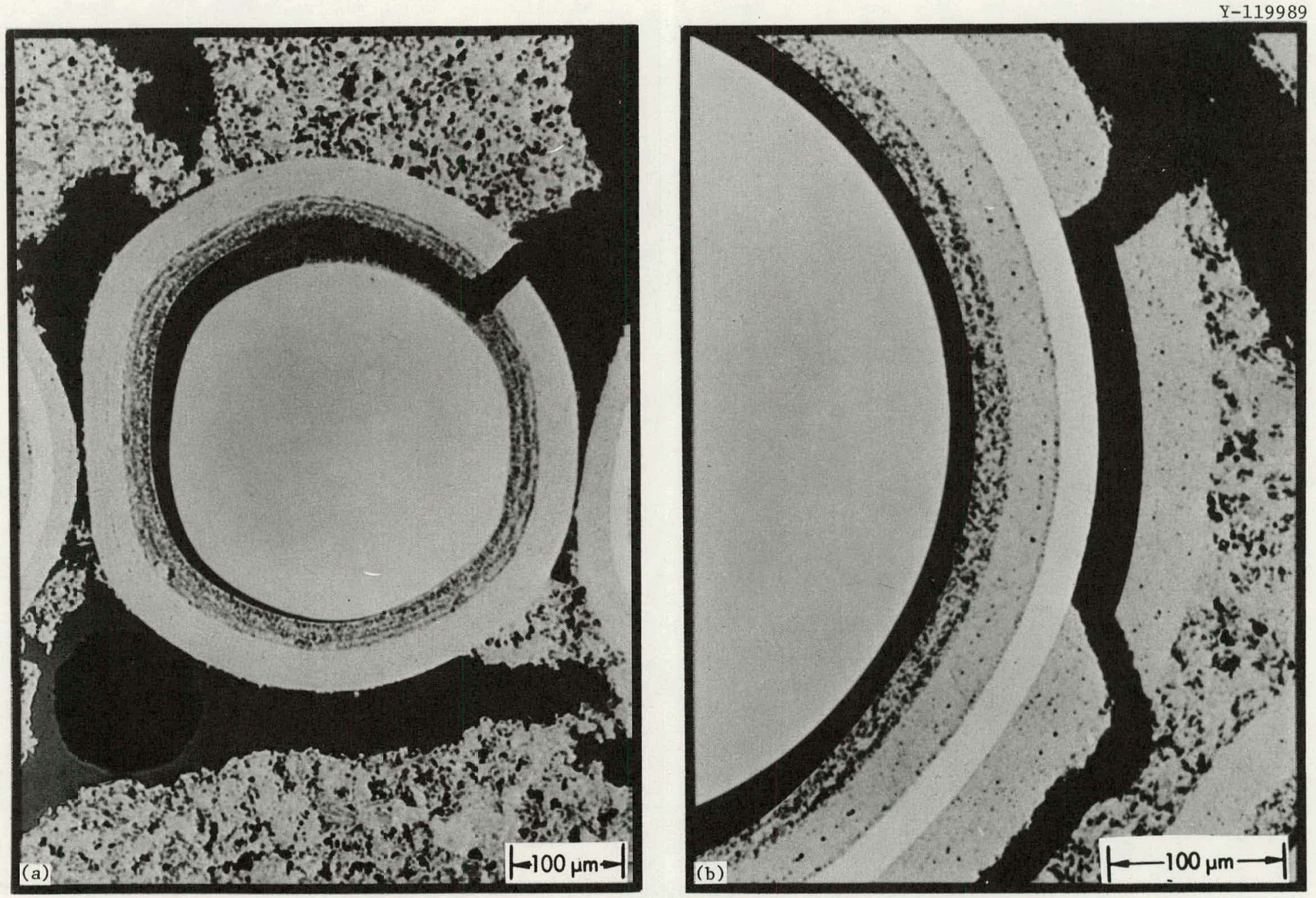

Fig. 23. Particles Broken During Irradiation Due to Matrix-Particle Interaction. Carbonized in graphite tube. Irradiated to $3.6 \times 10^{21} \mathrm{n} / \mathrm{cm}^{2}(\mathrm{E}>0.18 \mathrm{MeV})$ at $940-1145^{\circ} \mathrm{C}$. (a) Biso particle. (b) Triso particle. 
The causes of matrix-particle interaction are not yet fully understood. It is known that no single parameter is controlling, rather, it is a combination of matrix density, microporosity content, and pitch-coke yield. A comparison of the matrix structures for fuel rods irradiated in the OF-1 experiment ${ }^{15}$ is shown in Fig. 24. No matrix-particle interaction problem was encountered in the fuel rods irradiated in $0 F-1$.

From a thermal conductivity standpoint it is desirable to have a dense matrix. Therefore, work has been done to adjust the matrix materials and processing variables so that fuel rods can be manufactured with dense matrices, yet maintaining a high proportion of porosity as microporosity, as shown in Fig. 25. The appearances of the matrices produced by different carbonization techniques are more similar in Fig. 25 than was the case in Fig. 24. No problems with matrix-particle interaction have been encountered since the $\mathrm{HRB}-4$ and -5 experiment; however, all in-block simulations have been conducted in graphite tubes. The characteristics of fuel rod matrices produced by in-block carbonization (on a production basis) are stil1 unknown.

Examples of the irradiation performance of coated particles with fabrication defects are shown in Fig. 26. Four types of defects are shown in this figure: a missing segment of the SiC layer, a missing buffer layer, a nonspherical particle, and anistropic coating properties. The particles shown in Fig. 26 are not all of the same type and are not from the same fuel rod. Figure 26(a) is a WAR Triso particle; the others are dense-oxide Biso particles. It is not the intent of Fig. 26 to compare coating designs, but rather to give examples of failure due to fabrication defects.

\section{FUTURE PLANS}

The recycle fissile particles that have been developed for HTGR recycle application are adequate to survive the irradiation conditions of any HTGR application currently under consideration, including gas turbine and process heat HTGRs, for irradiation temperatures up to $1400^{\circ} \mathrm{C}$. Further improvements in this design of these particles can be made to enhance the performance of other portions of the fuel system, notably the fuel rods and fuel elements. Fuel development work nver the next few years can be divided into three categories:

1. development and irradiation testing in support of product specification development,

2. verification of the good performance of candidate fuels under realtime HTGR conditions, and

3. development of advanced fuels for gas turbine and process heat application. This work is primarily coating development and testing and improvements in the fuel rod to take advantage of the superior performance capabilities of the WAR kernels. 


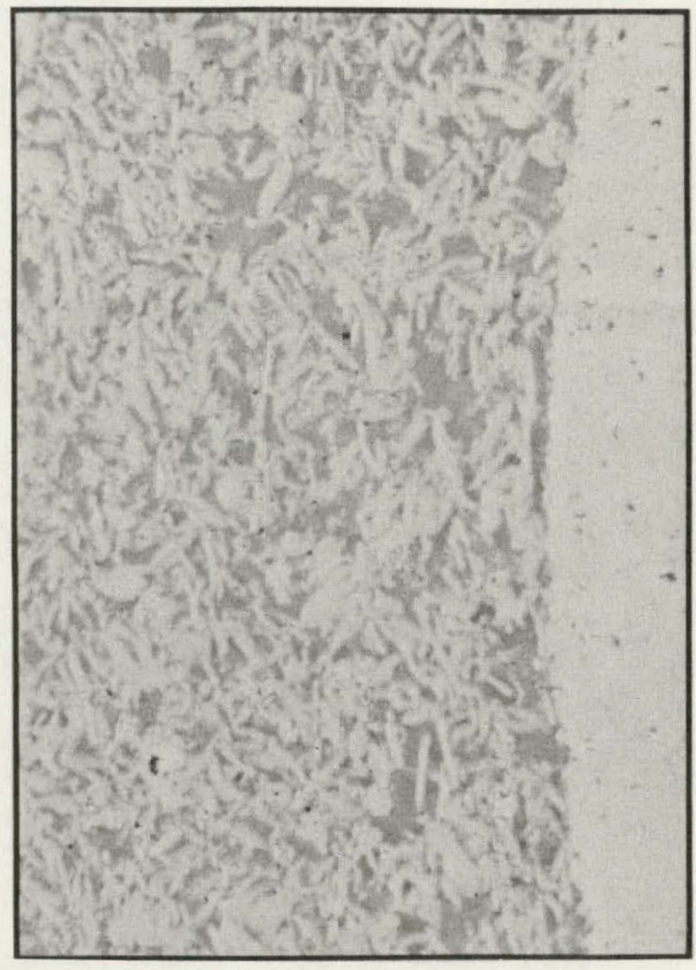

IN-PACKED ALUMINIA
13.7
41.6
0.473 PITCH COKE
YIELD (\%) MICROPOROSITY

$\begin{array}{ll}\text { (\%) } & 8.3 \\ \begin{array}{c}\text { DENSITY } \\ \mathrm{g} / \mathrm{cm}^{3}\end{array} & 0.732\end{array}$

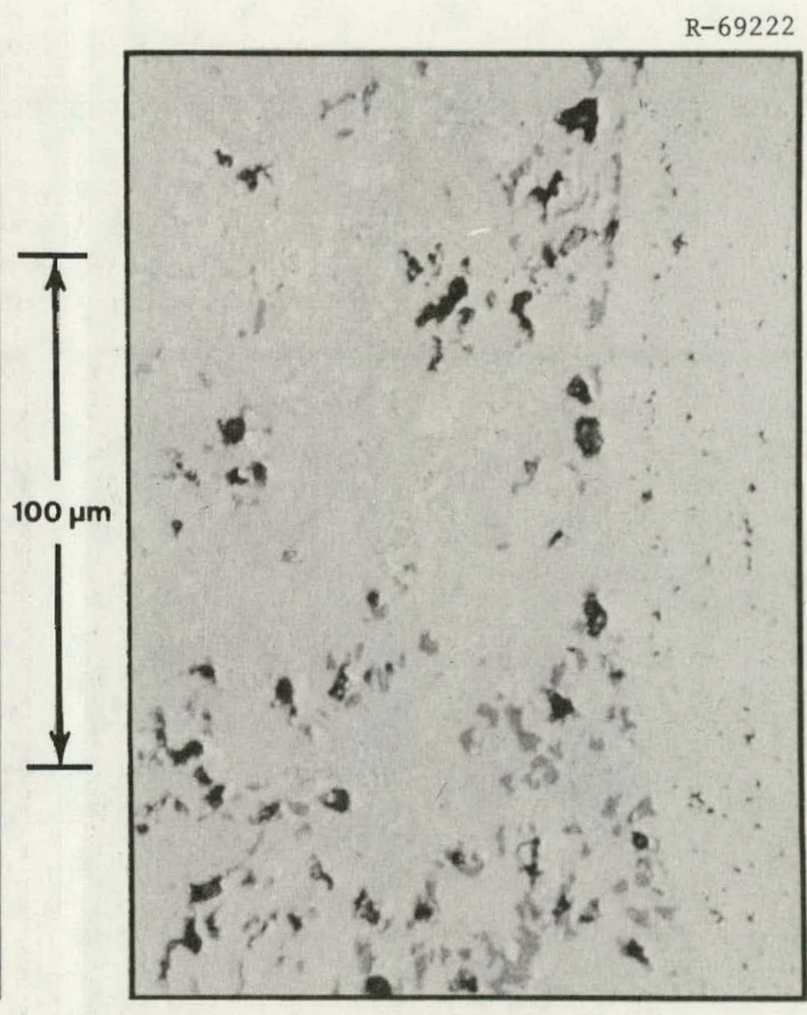

CARBONIZATION IN-BLOCK MODE

Fig. 24. Matrix Microstructures of Fuel Rods Irradiated in OF-1 Containing Asbury 6353 Filler (29 wt \%) and Ashland A240 Pitch. 
$\mathrm{Y}-119990$

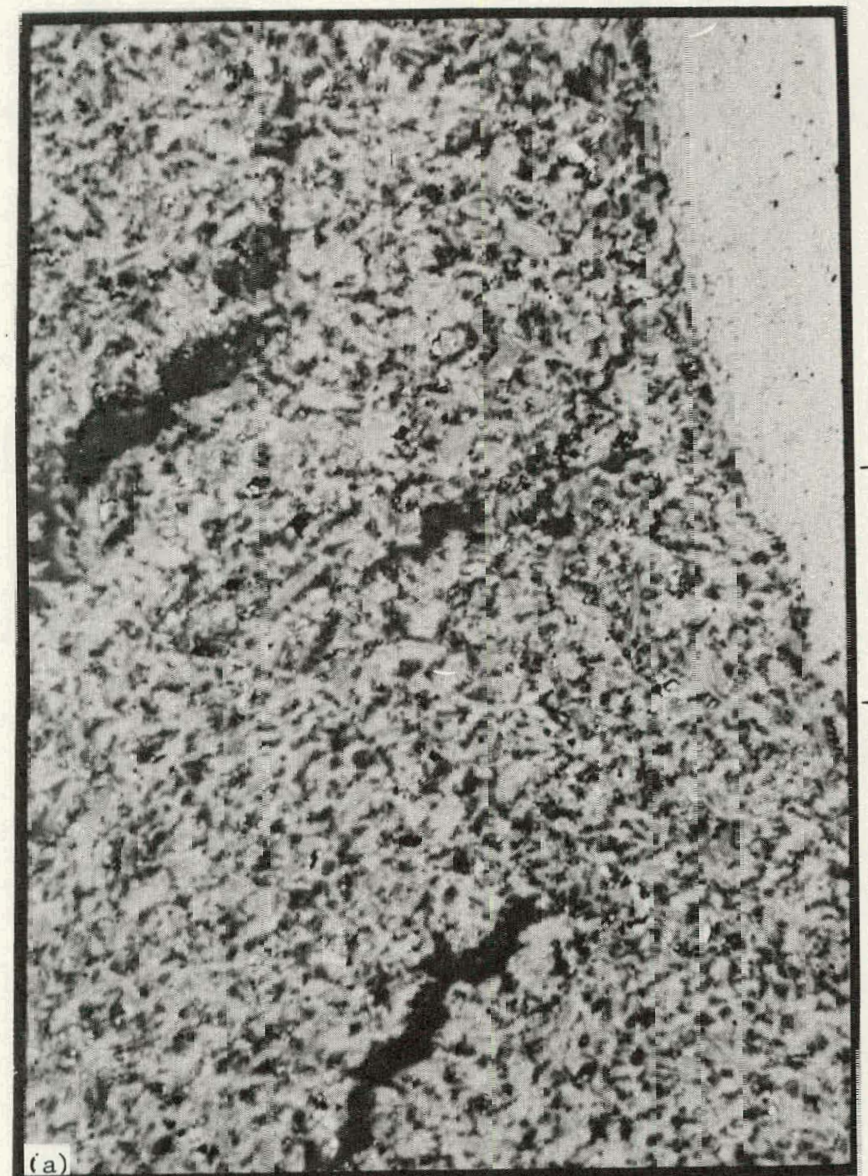

Packed $\mathrm{Al}_{2} \mathrm{O}_{3}$

30
0.75

Zarbonization Technique Microporosity $\vDash$ vol \%)

Matrix Density $\mathrm{g} / \mathrm{cm}^{3}$ )

26 Pitch Coke Yield (wt \%) 39

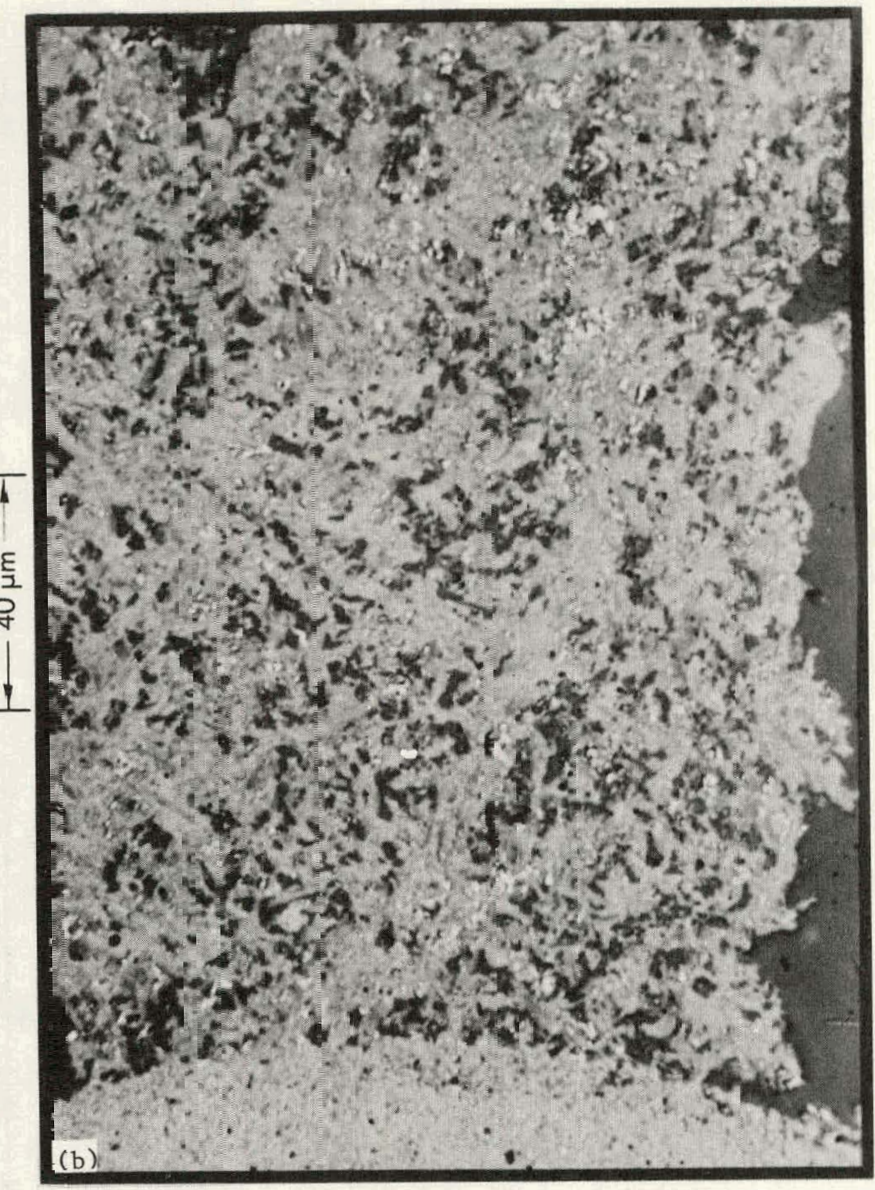

Graphite Tube

24

0.85

Fig. 25. Effect cf Matrix Microstructure on Zarticle Breakage. particles. (b) Particles broken.

(a) No broken 

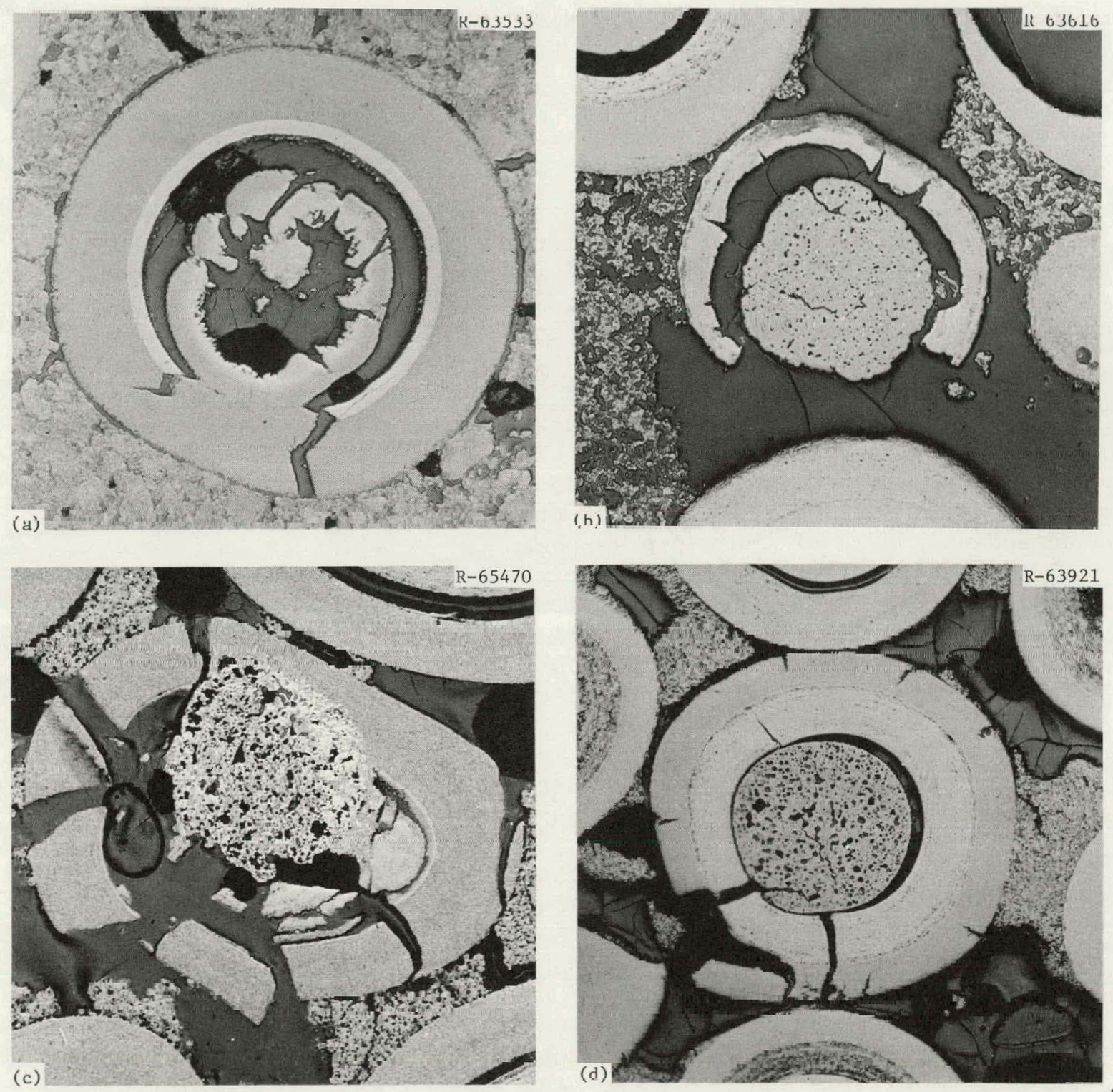

Fig. 26. Examples of Particles That Failed Because of Manufacturing Defects. 150x. (a) Portion of SiC missing. (b) Buffer missing. (c) Nonspherical particle. (d) Poor-coating properties. 
Product Specification Support

Over the next few years the product specification work will center around irradiation testing in the HFIR and the Oak Ridge Research Reactor (ORR). Approximately four HFIR capsules per year and one ORR capsule every two years will be dedicated to establishing product specifications.

\section{Real-Time Testing}

A11 data collected to date on the irradiation performance of WARderived fuels have been from accelerated tests. HRB tests reach full fast-neutron exposure in about ten months, compared to four years in an LHTGR and six years in the Fort St. Vrain Reactor (FSVR). Tests in the ORR reach full exposure in about 15 months, while HFIR target (HT) capsule Lests are fully exposed in about four months. Testing under accelerated conditions is believed to be conservative in that the fuel is subjected to higher power production rates (watts/particle) and higher temperature gradients than those listed in Tahle 1. However, there are a number of kinetic aspects to fuel performance, notably amoeba and fission product attack of the coatings. Since the timedependent nature of these processes is not established, the good performance of these fuels must be verified under real-time conditions. The Peach Bottom recycle test elements (RTE) ${ }^{14,16,17}$ were irradiated under HTGR conditions, but these elements received less than half the maximum neutron exposure and burnup anticipated for the T.HTGR, and no WAR fuel was included in these tests. Many of the performance characteristics observed for other fuels $\left[\mathrm{ThO}_{2},(\mathrm{Th}, \mathrm{U}) \mathrm{O}_{2}, \mathrm{UC}_{2}\right]$ in the RTEs have also been observed in these same fuels when irradiated under accelerated conditions. These observations provide confidence that accelerated testing data are relevant and that fuels that perform well under accelerated conditions will also perform well under real-time HTGR conditions. However, this point needs to be proven more convincingly by a direct comparison with current reference fuels irradiated to full burnup and fast-neutron exposure.

Plans for a direct comparison between $15-$ and $75 \%$-converted WAR fuel, both irradiated in the ORR and in FSVR, have been made. The fuel for this experiment is already under irradiation in the OF-2 capsule. in ORR. ${ }^{10}$ Fissile fuel from the same batches will be irradiated in FSVR test clcments 2,4 , and 6 , present1y scheduled to begin irradiation in the fall of 1977. Additional capsule tests (accelerated) and realtime tests (in FSVR) are scheduled in support of the cold prototype development and hot subsystems testing portions of the Thorium Utilization Program. 
Advanced Fuels

Beginning in FY 1977, work will begin at ORNL on advanced fuel designs to exploit the performance capabilities of WAR kernels. This work will concentrate on minimizing the temperature difference between the kernels and the coolant. Development will include the testing of alternate coatings, of thinner and stronger coatings, of higher conductivity matrices, and of alternate processing conditions that will produce fuel rods of higher overall thermal conductivity.

\section{DISCUSSION AND SUMMARY}

Five failure mechanisms have been identified and discussed. Of these mechanisms, two are independent of kernel chemistry and design. Amoeba, a severe problem with dense-oxide fissile kernels, has not been a problem with carbide fuels in the temperature and temperature-gradient regions of interest for the HTGR. Amoeba has not been observed in WAR-derived fuels containing more than $15 \% \mathrm{UC}_{2}$. Chemical interaction, a severe problem for dense carbide fuels, has been controlled in WAR fuels by the proper proportioning of $\mathrm{UO}_{2}$ and $\mathrm{UC}_{2}$ content. Pressure vessel failure due to dimensional changes in the coatings is independent of kernel type; however, the WAR fuel is considerably less dense than dense oxide or carbide kernels $\left(3 \mathrm{~g} / \mathrm{cm}^{3}\right.$ for WAR fuel vs $10 \mathrm{~g} / \mathrm{cm}^{3}$. for dense oxide and $13 \mathrm{~g} / \mathrm{cm}^{3}$ for dense carbide fuel). Also, WAR kernels have been observed to shrink during irradiation, leaving a gap between the kernel and the coatings. This high-porosity kernel and the void between the kernel and coatings will surely combine to raise the kernel operating temperatures of the WAR fuel (relative to dense kernels at the same watt/particle). The high temperature and high porosity will lead to higher fission gas release and higher stresses on the coatings, which may result in the need to redesign the coatings and make them thicker.

Lindemer'3 thermodynamic analyses' suggest that kernel stoichiometries ranging from 15 to $70 \%$ conversion from oxide to carbide should pertorm well. In kernels with less than $15 \%$ carbide, the fission-released oxygen will react with carbon to form $\mathrm{CO}$, which will raise the internal gas pressure and perhaps contribute to thermal migration as well as pressure vessel failure. Should the $C O$ reach the SiC layer due to failure or permeability of the inner $\mathrm{PyC}$, the $\mathrm{CO}$ will react with the SiC to form silfcon oxides and ultimately fail the SiC layer. In kernels with more than $70 \%$ carbide, there is insufficient oxygen present to combine with the chemically active rare-earth fission products, which will migrate as carbides and attack the SiC and inner PyC layers. Irradiation testing to date supports the conclusions of the thermodynamic studies. Stoichiometries of $0,15,50,75$, and $100 \%$ carbide have been tested. The WAR $\mathrm{UO}_{2}$ fuel probably exhibited excessive thermal migration. The WAR $\mathrm{UC}_{2}$ fuel exhibited excessive fission product attack of the SiC layer causing a high failure fraction in these particles. 
The 15, 50, and 75\% carbide kernels performed well, with increasing rare-earth fission product loss from the kernels (attack of the SiC noted) as carbide content increased.

The kernel process development work done to date has indicated that the flexibility anticipated for WAR fuel, with respect to kernel density and stoichiometry, is indeed possible in laboratory-scale equipment. The technology appears to be capable of extrapolation to production-scale equipment with no difficulty. Additional work on process development will continue to determine process latitudes.

The importance of the work described in this repore is that a fissile fuel has been developed which will perform well in any HTGR application currently under consideration - up to irradiation temperatures of $1400^{\circ} \mathrm{C}$. It is anticipated that additional irradiation tesling of optimum stoichiometries will further raise the temperaturc limit. Further, the processes required to produce these particles are readily adaptable to remote operation, which is essential for a recycle fuel. Direct comparisons of WAR-derived fuel with current reference fresh fuel (dense $U_{2}$ ) suggest superior performance by the WAR fuel, which further suggests that the WAR fuel may become the reference fresh fissile fuel as well as the reference recycle fuel.

Future work should be directed at optimizing the WAR particle design and developing a product specification for manufacture. Additional work should be done to improve the fuel rod and fuel element design to take advantage of the performance capabilities of the coated particles. Design modifications that minimize the temperature difference between kernels and coolant will permit higher coolant temperatures, higher thermal efficiency, and more complete capabillties of the HTGR relative to the needs of steam cycle power production and process heat applications.

\section{ACFNOWLEDGMTNT3}

The authors wish to acknowledge the entire HTGR staff for the significant contributions in the areas of planning, execution, and evaluation of the numerous irradiation experiments over the past decade. In particular, the authors wish to acknowledge P. R. Kasten, J. H. Coobs, A. L. Lotts, W. P. Fatherly, J. L. Scott, J. D. Sease, R. A. Bradley, R. B. Fitts, A. R. Olsen, and R. L. Beatty of the Metals and Ceramics Division and J. A. Conlin, B. H. Montgomery, and K. R. Thoms of the Reactor Division for their participation in the planning and designing of the experiments; the individuals in the Metals and Ceramics Division that participated in the fabrication and characterization of the test particles and fuel rod specimens: R. L. Hamner, J M Robbins, W. J. Lackey, A. J. Caputo, G. W. Weber, C. B. Pollock, W. H. Miller, Jr., C. Hamby, D. E. Rosson, J. B. Flynn, and C. E. DeVore. The dosimetry and thermometry of the capsules were provided by $H$. T. Kerr of the Reactor Division and R. L. Shepard of the Instrumentation and Controls Division, respectively. 
Thanks go to T. N. Tlegs and E. L. Ryan of the Metals and Ceramics Division for the conduction of the postirradiation examinations of the more recent experiments and to E. M. King and his staff of the Operations Division for their assistance in providing the facilities and services required to conduct postirradiation examinations. The metallography of the unirradiated controls was performed by M. D. Allen of the General Metallography group, and the remote metallography was performed by N. M. Atchley and L. G. Shrader of the Radiation and Electron Metallography group of the Metals and Ceramics Division. The electron microprobe analyses were performed by T. J. Henson of the Radiation and Electron Metallography group of the Metals and Ceramics Division. The authors also acknowledge the technical reviews and constructive comments provided by $T$. B. Lindemer and G. W. Weber, and the assistance of R. H. Powell, Jr., for technical editing and Julia Bishop for typing and makeup of the report.

\section{REFERENCES}

1. F. J. Homan et al., "Development of a Recycle Fuel for the HTGR," paper submitted to Nuclear Technology.

2. J. A. Conlin et al., "HTGR Fuel Irradiations and Postirradiation Examinations," pp. 90-97 in Gas-Cooled Reactor Progrons Annu. Progr. Rep. Dec. 31, 1973, ORNL-4975.

3. J. D. Sease, "Fuel Fabrication Process Development," pp. 33-56 in GasCooled Reactor Progroms Annu. Progr. Rep. Dec. 31, 1972, ORNL-4911.

4. Preliminary Safety Analysis Report for Fulton Generating Station, Chap. 4.

5. Preliminary Safety Analysis Report for Summit Generating Station, Chap. 4.

6. T. B. Lindemer and R. L. Pearson, Kernel Migration for H'l'GK Fuels from the (Th-U-C-O-N) System, ORNL/TM-5027 (April 1976).

7. 0. M. Stansfield et al., "Kernel Migration in Coated Carbide Fuel Particles," Nucl. Technol. 25: 517-30 (1975).

8. National HTGR Fuel Recyele Devilopment Program Plan, prepared by Oak Ridge National Laboratory and Gulf General Atomic, ORNL-4702 (August 1971), p. 16 .

9. J. L. Scott et a1., An Irradiation Test of Bonded HTGR Coated Particle Fuels in an Instmmented Capsule in HFIR, ORNL-TM-3640 (March 1972).

1.0. R. A. Olstad et al., An Irradiation Test of Candidate HTGR Recycle Fuels in the $\ddot{H}-1$ and $H-2$ Capsules, ORNL-TM-1397 (.T11.y 1974;

Addendum, April 1975). 
11. K. H. Valentine et al., Irradiation Performance of HTGR FueZ Rods in HFIR Experiments $H R B-7$ and -8 , in preparation.

12. F. J. Homan et al., Irradiation Performance of HTGR Fuel Rods in HFIR Experiments HRB-4 and -5, ORNL-5115 (June 1976).

13. F. J. Homan et al., Irradiation Performance of HTGR Fuel Rods in HFIR Experiments $H R B-9$ and -10 , in preparation.

14. E. L. Long, et al., Fabrication of ORNL Fuel Irradiated in the Peach Bottom Reactor, and. Postimradiation Exurination of Recycle Test Elements 7 and 4, ORNL-TM-4477 (September 1974).

15. T. N. Tiegs et al., Irradiation Performance of HTGR Fuel Rods in $O R R$ Experiment $O F-1$, in preparation.

16. R. B. Fitts, "Recycle Test Elements in the Peach Bottom Reactor," pp. 126-33 in Gas-Cooled Reactor Frograns Annu. Progr. Rep. Der. 31, 1972, ORNL-4911.

17. J. A. Conlin et a1., "HTGR Fuel Irradiations and Postirradiation Examinations," pp. 89-172 in Gas-Cooled Reactor Progroms Annu. Progr. Rep. Dec. 31, 1973, ORNL-4975.

18. HTGR Base Program Progress Report for January 1, 1974, through June 30, 7.975, ORNL-5108, Cliap. 6 in preparation. 


\section{ORNL/TM-5502 \\ UC-77 (Gas-Cooled \\ Reactor Technology)}

\section{INTERNAL DISTRIBUTION}
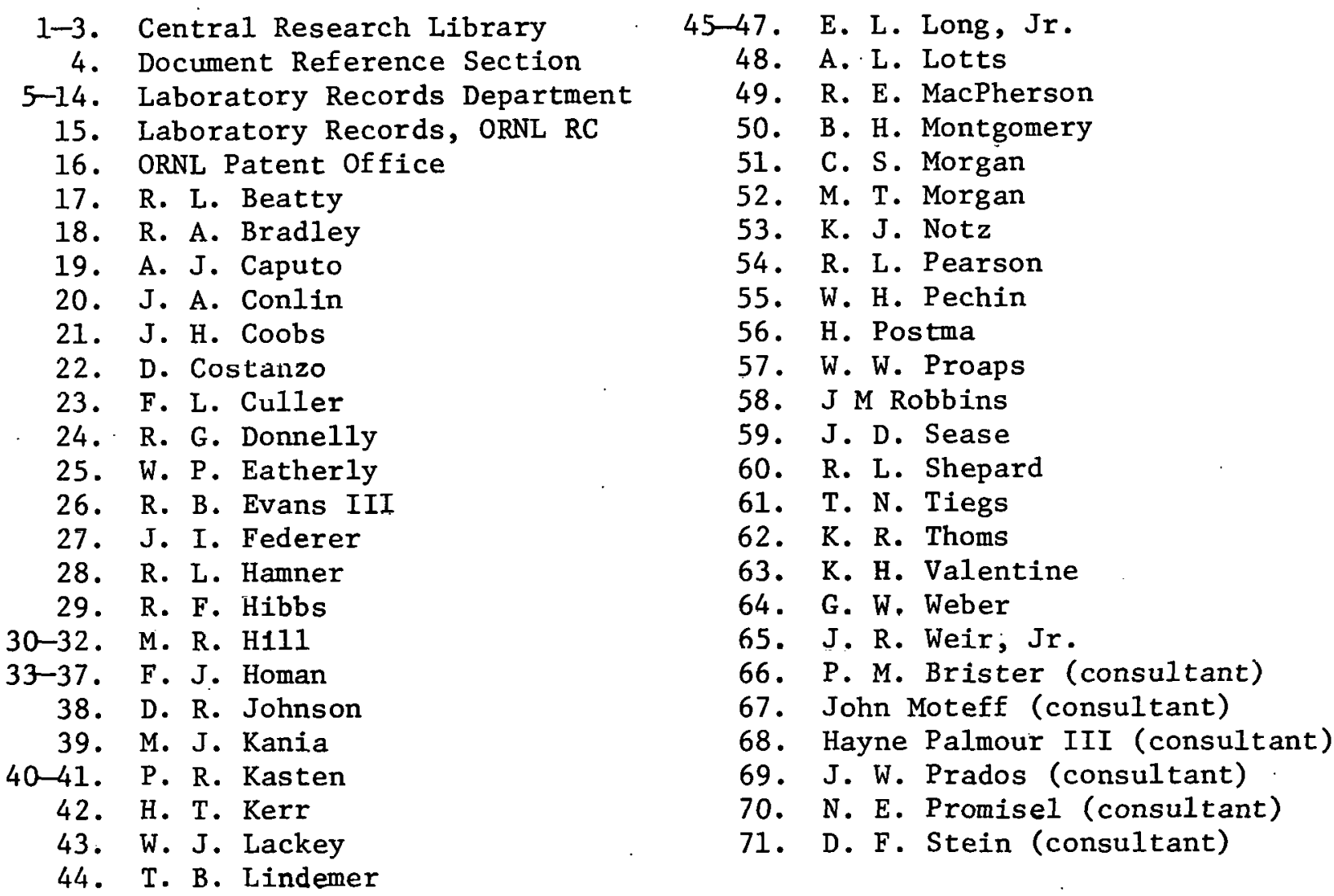

EXTERNAL DISTRIBUTION

72-73. ERDA DIVISION OF NUCLEAR FUEL CYCLE AND PRODUCTION, Washington, DC 20545

Director

74-75. ERDA DIVISION OF NUCLEAR RESEARCH AND APPLICATION, Washington, DC 20545

Director

76-77. ERDA OAK RIDGE OPERATIONS OFFICE, P.O. Box E, Oak Ridge, TN 37830

Director, Reactor Division

Director, Research and Technical Support Division

78-244. ERDA TECHNICAL INFORMATION CENTER, Office of Information Services, P.0. Boys 63, Dak. Ridge, 'I'N 37830

For distribution as shown in TID-4500 Distribution Category, UC-77 (Gas-Cooled Reactor Technology) 